\title{
Processes of consent in research for adults with impaired mental capacity nearing the end of life: systematic review and transparent expert consultation (MORECare_Capacity statement)
}

\author{
C. J. Evans ${ }^{1,2^{*}}$ (1) E. Yorganci ${ }^{1}$, P. Lewis ${ }^{3}$, J. Koffman ${ }^{1}$, K. Stone ${ }^{1}$, I. Tunnard ${ }^{1}$, B. Wee ${ }^{4}$, W. Bernal ${ }^{5}$, M. Hotopf ${ }^{6}$, \\ I. J. Higginson ${ }^{1}$ and on behalf of MORECare_Capacity
}

\begin{abstract}
Background: Involving adults lacking capacity (ALC) in research on end of life care (EoLC) or serious illness is important, but often omitted. We aimed to develop evidence-based guidance on how best to include individuals with impaired capacity nearing the end of life in research, by identifying the challenges and solutions for processes of consent across the capacity spectrum.

Methods: Methods Of Researching End of Life Care_Capacity (MORECare_C) furthers the MORECare statement on research evaluating EoLC. We used simultaneous methods of systematic review and transparent expert consultation (TEC). The systematic review involved four electronic databases searches. The eligibility criteria identified studies involving adults with serious illness and impaired capacity, and methods for recruitment in research, implementing the research methods, and exploring public attitudes. The TEC involved stakeholder consultation to discuss and generate recommendations, and a Delphi survey and an expert 'think-tank' to explore consensus. We narratively synthesised the literature mapping processes of consent with recruitment outcomes, solutions, and challenges. We explored recommendation consensus using descriptive statistics. Synthesis of all the findings informed the guidance statement.

\footnotetext{
* Correspondence: catherine.evans@kcl.ac.uk

${ }^{1}$ Cicely Saunders Institute of Palliative Care, Policy \& Rehabilitation, Florence

Nightingale Faculty of Nursing, Midwifery \& Palliative Care, King's College London, Bessemer Road, London SE5 9PJ, UK

${ }^{2}$ Sussex Community NHS Foundation Trust, Brighton General Hospital, Brighton, UK

Full list of author information is available at the end of the article
}

(c) The Author(s). 2020 Open Access This article is licensed under a Creative Commons Attribution 4.0 International License, which permits use, sharing, adaptation, distribution and reproduction in any medium or format, as long as you give appropriate credit to the original author(s) and the source, provide a link to the Creative Commons licence, and indicate if changes were made. The images or other third party material in this article are included in the article's Creative Commons licence, unless indicated otherwise in a credit line to the material. If material is not included in the article's Creative Commons licence and your intended use is not permitted by statutory regulation or exceeds the permitted use, you will need to obtain permission directly from the copyright holder. To view a copy of this licence, visit http://creativecommons.org/licenses/by/4.0/ The Creative Commons Public Domain Dedication waiver (http://creativecommons.org/publicdomain/zero/1.0/) applies to the data made available in this article, unless otherwise stated in a credit line to the data. 


\begin{abstract}
(Continued from previous page)
Results: Of the 5539 articles identified, 91 met eligibility. The studies encompassed people with dementia (27\%) and in palliative care (18\%). Seventy-five percent used observational designs. Studies on research methods (37 studies) focused on processes of proxy decision-making, advance consent, and deferred consent. Studies implementing research methods (30 studies) demonstrated the role of family members as both proxy decisionmakers and supporting decision-making for the person with impaired capacity. The TEC involved 43 participants who generated 29 recommendations, with consensus that indicated. Key areas were the timeliness of the consent process and maximising an individual's decisional capacity. The think-tank $(n=19)$ refined equivocal recommendations including supporting proxy decision-makers, training practitioners, and incorporating legislative frameworks.

Conclusions: The MORECare_C statement details 20 solutions to recruit ALC nearing the EoL in research. The statement provides much needed guidance to enrol individuals with serious illness in research. Key is involving family members early and designing study procedures to accommodate variable and changeable levels of capacity. The statement demonstrates the ethical imperative and processes of recruiting adults across the capacity spectrum in varying populations and settings.
\end{abstract}

Keywords: Palliative care, Terminal care, Decision-making, Consent, Methods, Ethics, Systematic review, Consensus

\section{Background}

There is an urgent need for evidence on best practice in palliative care. The projected increases in global serious health-related suffering demand immediate action. By 2060, an estimated 48 million people will die globally with serious related-suffering, representing an $87 \%$ increase from the 26 million in 2016 [1]. Failing to respond will see $80 \%$ of people globally with little or no access to palliative care services and treatment [2]. A major barrier in research on palliative care is ethical concerns about the perceived vulnerability of adults with serious illness and including them in research, particularly if the person also has impaired mental capacity [3]. Exclusion of adults with impaired capacity to consent for themselves impedes evidence-based care and treatment that is applicable across the illness trajectory and end of life (EoL) [4]. New interventions require robust evaluation to examine benefit and potential of harm for the population intending to benefit $[5,6]$. Studies, especially clinical trials in palliative care, are often compromised by insufficient sample size to detect change [713], and impaired understanding of legislation governing research involving adults with impaired capacity [14]. The ethical challenges of recruiting individuals with impaired capacity are examined across fields involving adults with serious illness including palliative care [15], dementia [16-18], mental health [19], and intensive care [20]. Systematic reviews have considered consent processes in specific conditions (e.g. dementia [21], schizophrenia [22]) and aspects of involving adults lacking capacity in research (e.g. capacity assessment [22], enhancing informed consent with older people [23, 24], and strategies for designing research studies [25] and increasing the recruitment rate in palliative care [26]). But, in palliative care, intervention studies are few and often exclude adults lacking capacity, for example in the dying phase [27]. There is literature from both within and outside the field of palliative care that could inform much needed guidance on best practice on processes of consent across the capacity spectrum in serious illness. This study aimed to determine how best to include individuals with impaired capacity in research on EoLC by identifying challenges for and solutions to processes of consent across the capacity spectrum. This paper reports the integrated results from a systematic review and transparent expert consultation to form the MORECare Capacity statement on processes of consent in research on EoLC. This furthers the Methods Of Researching End of Life Care (MORECare) statement on evaluating complex circumstances in EoLC [28] by giving detailed consideration on processes of consent for adults with serious illness across the capacity trajectory. The MORECare statement omitted this area, focusing on outcome measurement, response shift and attrition, integrating mixed methods and economic evaluation.

In this paper, 'capacity' refers to mental capacity to make an informed decision regarding research participation. 'The spectrum of capacity' of individuals ranges from potentially impaired, and anticipated to have impaired capacity, to lacking capacity. The legislation governing involvement of adults lacking capacity in research and terminology is jurisdiction specific. In this paper, the term 'consultee' (someone who has capacity) is used to encompass the different terms used in respective jurisdictions including but not limited to proxy-decision maker, personal consultee and nominated consultee. A distinction is made between a personal consultee (e.g. family member) and a nominated consultee (e.g. health 
professional) [29]. 'The process of consent' refers to the steps taken to ensure that an eligible research participant is sufficiently informed about the purposes, content, affiliations of the study, and their right to withdraw from the study at any point, enabling them or their consultee to decide freely about research participation [30].

\section{Methods}

\section{Study design}

We used a parallel iterative research design detailed in Fig. 1. We used methods of systematic literature review to identify and map the challenges and solutions for processes of consent for adults with impaired capacity, and the MORECare transparent expert consultation (TEC) to debate key areas [28] of uncertainty/contention. The TEC involved expert stakeholder consultations using consensus methods of modified nominal group technique to generate recommendations [31], and then presenting the recommendations in an online Delphi survey to explore levels of agreement [32]. We held a final expert think-tank to explore areas of contention/uncertainty and synthesise the findings to develop the statement. King's College London Research Ethics Committee approved the TEC component (ref no. BDM/10/11-90).

\section{Systematic review}

\section{Design}

We used systematic review method of narrative synthesis to systematically identify, appraise, and synthesise quantitative and qualitative literature [33]. Methods of analysis and inclusion criteria were pre-defined in the study protocol. Reporting followed the PRISMA guidance [34] (see Additional file 1: Table S1).

\section{Eligibility criteria}

Population Adults ( $\geq 18$ years old) with impaired capacity encompassing declining capacity (e.g. mild to moderate dementia), fluctuating capacity (e.g. delirium), and lack of capacity (e.g. dying, advanced dementia) are included.

Context The scoping of the literature identified areas recruiting adults lacking capacity with serious illness in research. We included studies from palliative care, mental health (delirium, dementia, learning disabilities), or emergency medicine/critical care.

Interest Studies discussing consent in its various forms (e.g. informed, advanced, proxy) and impaired mental capacity are included. We did not restrict by health or behaviour outcome. We focused on research studies investigating either of the following: (i) methods for involving adults with impaired capacity in research, (ii) implementing research methods to enable recruitment, or (iii) exploring public attitudes and ethical issues on involvement in research.

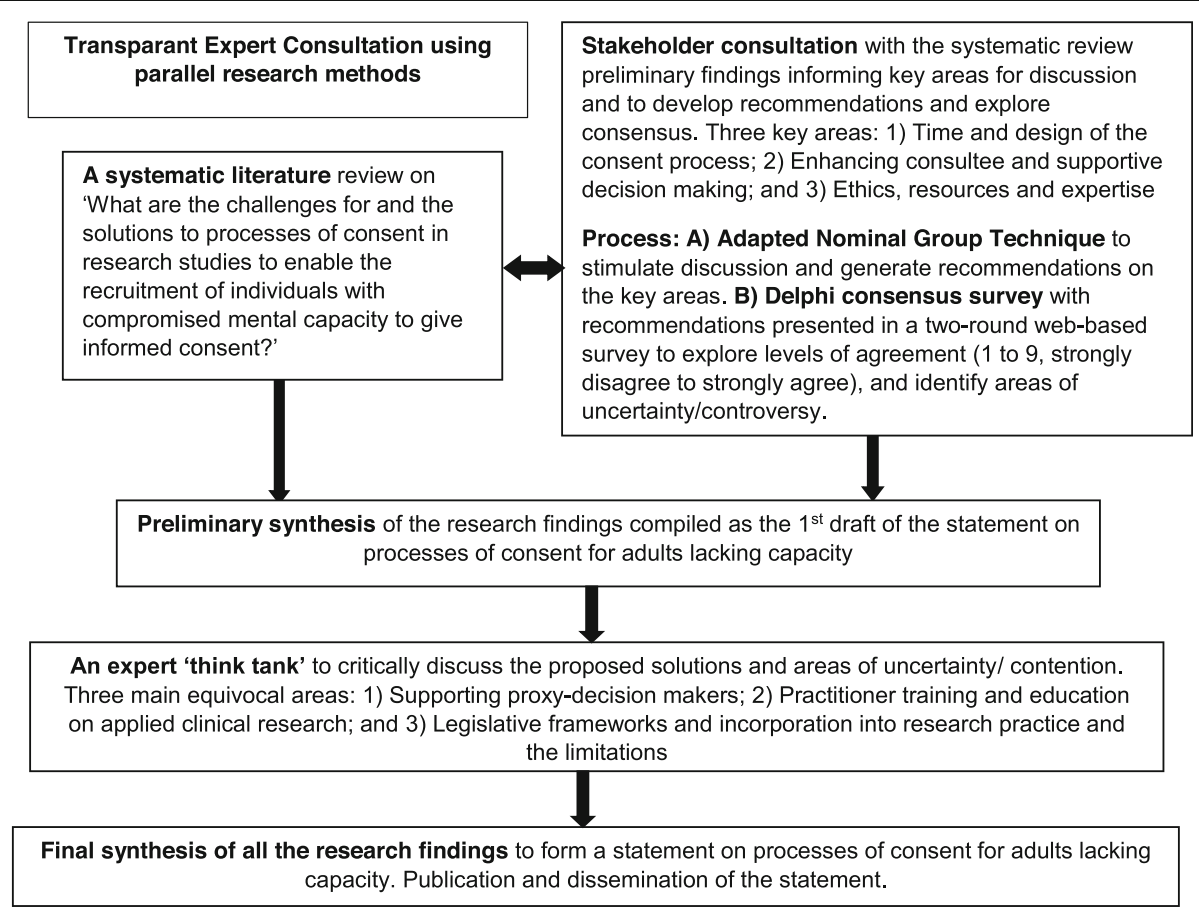

Fig. 1 Overview of the study design 
Design Randomised controlled trials (RCTs) and quasiexperimental, mixed method, or observational qualitative or quantitative designs are included. We included published study protocols that reported the methods of consent to enhance understanding for the main study. We excluded systematic reviews but used relevant systematic reviews for reference chaining by screening the cited publications for eligibility. We excluded opinion pieces and commentaries and non-English language papers. Studies concerning treatment/clinical decision-making or bioethics were out of scope.

\section{Search strategy}

We developed search strategies for each of the population groups-palliative care, mental health (including dementia), and emergency medicine/critical care. MeSH terms for the palliative care group included 'Terminally ill' or 'Palliative Care' AND 'Decision making' or 'Mental Competency' AND 'Informed consent' or 'Third-party consent'. Key search terms were used as free-text, and with use of truncation symbol to retrieve variations in the terminology. Search terms were piloted pre-study and mapped to assess their relevance and specificity, and refined working with a specialist librarian (see Additional file 2: Table S2 - electronic search terms). We searched four electronic databases: MEDLINE (1966Present), EMBASE (1947-Present), PsychINFO (1887Present), and CINAHL (1937-Present), and supplemented with referencing chain, grey literature electronic survey, and expert recommendations. The last search was run on 30 October 2018.

\section{Quality appraisal}

Study risk of bias was assessed using the validated QualSyst review tool suitable for quantitative and qualitative studies [35] by one reviewer (EY, IT, CJE), and a random $10 \%$ sample checked by a second author (CJE). Scores that diverged by $>10 \%$ were discussed within the research team. A single quality appraisal was undertaken for studies reported in multiple publications (e.g. protocol paper and a main trial results paper). The QualSyst assessment criteria include 14 items for quantitative studies and 10 items for qualitative studies. Each item is scored from 0 to 2 ( 0 , not present; 1 , partial; 2 , yes; or not applicable). The percentage of the total possible score indicates the quality grade: $<50 \%$, low; $\geq 50$ and $<70 \%$, medium; or $\geq 70 \%$, high. Study designs were categorised using the Cochrane Effective Practice and Organisation of Care (EPOC) grade system [36] (see Additional file 2: Table S3).

\section{Data screening, extraction, and analysis}

Referencing software (Endnote version x8) [37] was used to manage a database of search findings and remove duplicates. Title and abstract screen by one reviewer $(\mathrm{EY} / \mathrm{KS})$ and second independent review of $20 \%$ to test the application of the eligibility criteria (CJE). Titles/abstracts that met the review criteria, or if insufficient information to determine eligibility, were subject to fulltext screening. Full-text articles were single-screened by three reviewers (EY/KS/CJE). Full-text papers with uncertain eligibility were reviewed by two reviewers and eligibility agreed (EY/KS with CJE). A standardised data extraction form was developed and piloted based on the Cochrane Consumers and Communication Revie Group's data extraction template (see Additional file 2: Table S4). Data included the study design and aim, the population and context, method(s) of consent, recruitment rate, and challenges and solutions. Study data were extracted by one reviewer (CJE, EY, KS) and checked by a second reviewer (CJE, EY, KS). We contacted two authors to check availability of the publications in English. Using narrative synthesis [33], textual descriptions from extracted data for all studies were mapped to form matrices for studies innovating research method, studies using innovative methods, or considered ethics, legislation, or public attitudes. Each matrix was analysed and coded in Microsoft Excel using thematic analysis to explore prominent themes. Higher quality studies were valued with a greater strength in the final synthesis.

\section{Transparent expert consultation}

The TEC aimed to enhance the systematic review findings by exploring the application of the findings in research studies and areas little considered or uncertain in the evidence. The TEC explored researchers' and service users' perspectives on recruiting individuals with impaired capacity in research on EoLC. The TEC sought to generate recommendations on processes of consent to enable recruitment and explore the level of consensus.

\section{Setting and participants}

Participants were purposively sampled based on their expertise in conducting research involving adults with impaired capacity (including ethicists), caring for patients with advanced disease, or a service user/carer (e.g. palliative care services), or a voluntary sector representative (e.g. Alzheimer's Society). Participants were invited to the workshops held in the Cicely Saunders Institute, King's College London. Eligible participants included members of the project's expert panel (project applicants), Project Advisory Group (invited experts in, for example, ethics, and PPI and voluntary sector representatives) [see the "Acknowledgements" section], respondents in the systematic review grey literature survey and invited ethicists, clinicians, commissioners, researchers, members of ethical committees, policymakers, and service user and lay voluntary sector representatives. 
Identified professional participants received email invitations for the workshop. Service user and lay voluntary sector representatives were recruited via voluntary sector groups including, for example, Alzheimer's Society and Independent Cancer Patients' Voice. The respective organisations circulated the invitation letter to their members targeting those known to have an interest/experience of either a carer for an adult with impaired capacity, being a patient with progressive illness, or supporting research involving adults with impaired capacity.

The TEC used four stages:

Stage I: Identifying critical issues. The initial workshop focused on critical issues identified from the systematic review preliminary findings and expert opinion (e.g. areas with limited empirical evidence and relevance in the processes of consent for adults across the capacity spectrum).

Stage II: Stakeholder workshop. Participants received a pre-workshop briefing pack detailing the aim, critical issues, and workshop format. The workshop comprised presentations on the critical areas overviewing findings from the systematic review followed by structured group discussion involving 10-14 participants focusing on one of the critical areas. Group discussions were digitally recorded. We used a structured nominal group process facilitated by a member of the research team. The facilitator guided participants through a structured process of (1) a brief discussion, (2) individual writing of recommendations and ranking, and (3) participants in turn stating their highest ranked recommendations until individual lists were exhausted (or time exceeded) [31]. Scribes wrote the recommendations and ranking on a flipchart, and each small group discussed and agreed on the final priority order, then presented and discussed with the whole group. Participants individually listed and ranked recommendations from one to five (highest to lowest) on structured A4 sheets detailing the respective group question, ranking scale and boxes to list recommendations, rank, and detail rationale.

Stage III: Delphi online consensus exercise. This is a two-round online consensus exercise [32]. Recommendations generated in the workshop were posted online to the workshop participants, members of the expert panel and Project Advisory Group, and respondents to the grey literature survey. Participants received a personalised email invitation and reminder after 2 weeks. The online participants anonymously ranked, from one to nine (strongly disagree to strongly agree), the extent they agreed with a recommendation and used free-text spaces to comment on each recommendation. Findings from round 1 informed requirements to revise recommendations where comments suggested ambiguity. Round 2 represented the revised recommendations and the median score for each recommendation from round 1 .
Participants again indicated their level of agreement ranked from one to nine and provided free-text commentary on, for example, rationale for ranking score.

Stage IV: Expert 'think-tank'. The expert 'think tank' workshop aimed to aid data synthesis and inform the solutions and recommendations in the statement by critically considering areas of contention/uncertainty identified in the consensus exercise and systematic review findings. The think-tank aimed to understand the debates surrounding these areas, the strengths and limitations of the evidence, and the solutions for practice. Participants were purposively selected from the workshop participants based on expertise, e.g. ethicists, lay voluntary sector representative, researcher, and clinician. Think-tank participations received a briefing report that summarised for the respective area the systematic review findings on the challenges and solutions identified in the evidence base, and the recommendations and level of agreement from the consensus exercise. The think-tank used a format of presentations and debate, drawing on structured nominal group process to facilitate participant agreement on the top two or three key solutions for each area, and commentary on their thinking. Participants discussed and debated these areas in groups of 6-7. Discussions were digitally recorded, and scribes recorded on flipchart the key debates.

\section{Data analysis}

Individual recommendations from the workshop and their ranking were entered in Excel spreadsheets with assigned participant identification numbers. Two researchers (CJE, KS) coded and arranged recommendations by themes, duplicates were combined, and recommendations arranged by priority ranking (1 highest to 5 lowest). Free-text comments were collated. Digital recordings were reviewed to inform understanding on the recommendations and debates presented, with key points noted on the Excel spreadsheet for the respective recommendation. The recommendations retained participants' original language where possible with amendments guided by the expert panel to enhance clarity and avoid repetition. The final recommendations were those ranked the highest $(\leq 3)$ and reviewed and agreed by the expert panel and piloted (e.g. for clarity), and then posted on the online consensus survey. Analysis of the consensus survey-scaled data used descriptive statistics (frequencies and medians) and plots (box and whisker plots) of interquartile ranges to analyse and interpret levels of agreement. We used a conventional categorisation to interpret agreement (indicated, equivocal, or not indicated) and strength of agreement (strict or broad) used in previous consensus studies [28]. Table 1 details the categories by the respective median region and IQR [38]. Narrative comments were collated by 
recommendation, and themes identified to understand the issues raised and provide illustrative examples [32].

\section{Results}

Systematic review search results

The electronic database searches identified 5539 abstracts after removal of duplicates with a further 179 publications identified from other sources (see Fig. 2). Ninety-one publications met the eligibility criteria, reporting 89 studies. Two studies included a protocol and main results papers [39-42]. Studies were conducted mainly in dementia $(n=23)$, palliative care $(n=$ 16), and intensive care $(n=15)$ (Table 2). Publications increased over time with the majority published after $2010(n=54)$. Studies were conducted mainly in the USA $(n=35)$, UK $(n=29)$, or Canada $(n=9)$ (see Table 2 and Table 3, and Additional file 3: Table S8). The studies formed three main areas of (1) innovating research methods to recruit adults across the capacity spectrum, (2) applying consent processes across the capacity spectrum in studies on serious illness, and (3) public attitudes on involving adults lacking capacity in research.

\section{Quality appraisal}

Overall, the quality of the included articles was medium to high. Most quantitative $(95.8 \%, n=71)$ and qualitative studies $(88.9 \%, n=17)$ were assessed as medium or high quality (see Additional file 2: Table S5 quantitative studies and Table S6 qualitative studies). The proportion of high-quality studies included was consistent across the three main areas (56.8\% 'innovating research methods', $56.6 \%$ 'applying consent processes', and 59.1\% 'public attitudes'). However, in the area of 'public attitudes on involving adults lacking capacity', 9\% were assessed as low quality, compared with $0 \%$ in 'innovating research methods' and $2 \%$ in 'applying consent processes'. This reflected in part the methodological nature of the studies and poorer fit with the Qualsyst item criteria. The included studies were mainly descriptive $(n=36)$ categorised as 'non-experimental, longitudinal, cohort, matched pairs, or cross-sectional, sound qualitative, or analytical studies', with few experimental $(n=20)$ or

Table 1 Levels of consensus and agreement by median regions and IQR [38]

\begin{tabular}{ll}
\hline Median regions and IQR & Interpretation \\
\hline $\mathbf{7 - 9}$ & Recommendation indicated \\
$\mathbf{4 - 6}$ & Recommendation equivocal \\
1-3 & Recommendation not indicated \\
IQR in one region & Strict agreement for recommendation \\
IQR in any three-point region & Broad agreement for recommendation \\
\hline IQR interquartile range &
\end{tabular}

quasi-experimental designs $(n=3)$ (see Additional file 3: Table S7).

\section{Innovating research methods to recruit adults across the capacity spectrum}

Thirty-seven studies were categorised as innovating research methods (Table 3). Studies focused on participation in research involving individuals with cancer/ receiving palliative care $(n=6)$, dementia $(n=13)$, geriatric care (all settings) $(n=2)$, delirium and mental health services $(n=7)$, or intensive care $(n=5)$. While numerous studies used standardised capacity assessment tools, existing tools were often regarded as time-consuming, and administration of a formal capacity assessment reduced recruitment, for example in an observational study involving patients with delirium [99]. Formal capacity assessment was considered of little value unless aligned to the decisional requirements for study participation, notably the risks and the potential direct or indirect benefits of participation. Overall, studies incorporated multiple components of the processes of consent. These were tailored to individuals' level of capacity from mild to moderate impairment with a focus on enhancing informed consent, through to lacking capacity requiring involvement of a consultee. For example, in populations such as psychiatric or stroke patients, where participants experienced varying levels of impaired capacity, studies incorporated processes of enhanced informed consent and consultee involvement $[87,90]$. In both studies, consultee advice was sought for a third of participants (30.6\% and 35.7\%, respectively). The innovations broadly mapped onto two sub-categories of 'maximise individuals' autonomy and decisional capacity in the consent process' and 'processes of consent to enable adults across the capacity spectrum to participate in research'.

\section{Maximise individuals' autonomy and decisional capacity in the consent process}

Fifteen studies aimed to examine ways to enhance the informed consent process to maximise decisional capacity for adults with mild/moderate capacity or fluctuating capacity (see Table 3). Clinical trials required applicable methods to facilitate understanding of complex procedures, e.g. randomisation and clinical equipoise. For populations with mild/moderate dementia, and other neuropsychological disorders such as Parkinson's disease, studies aimed to enhance various facets of cognition. This involved in the informed consent, for example, enhancing decisional capacity, understanding [90], reasoning, comprehension, and recall of information. Across patient groups, key challenges to enabling participation in the informed consent process were addressing concerns about causing distress for the 


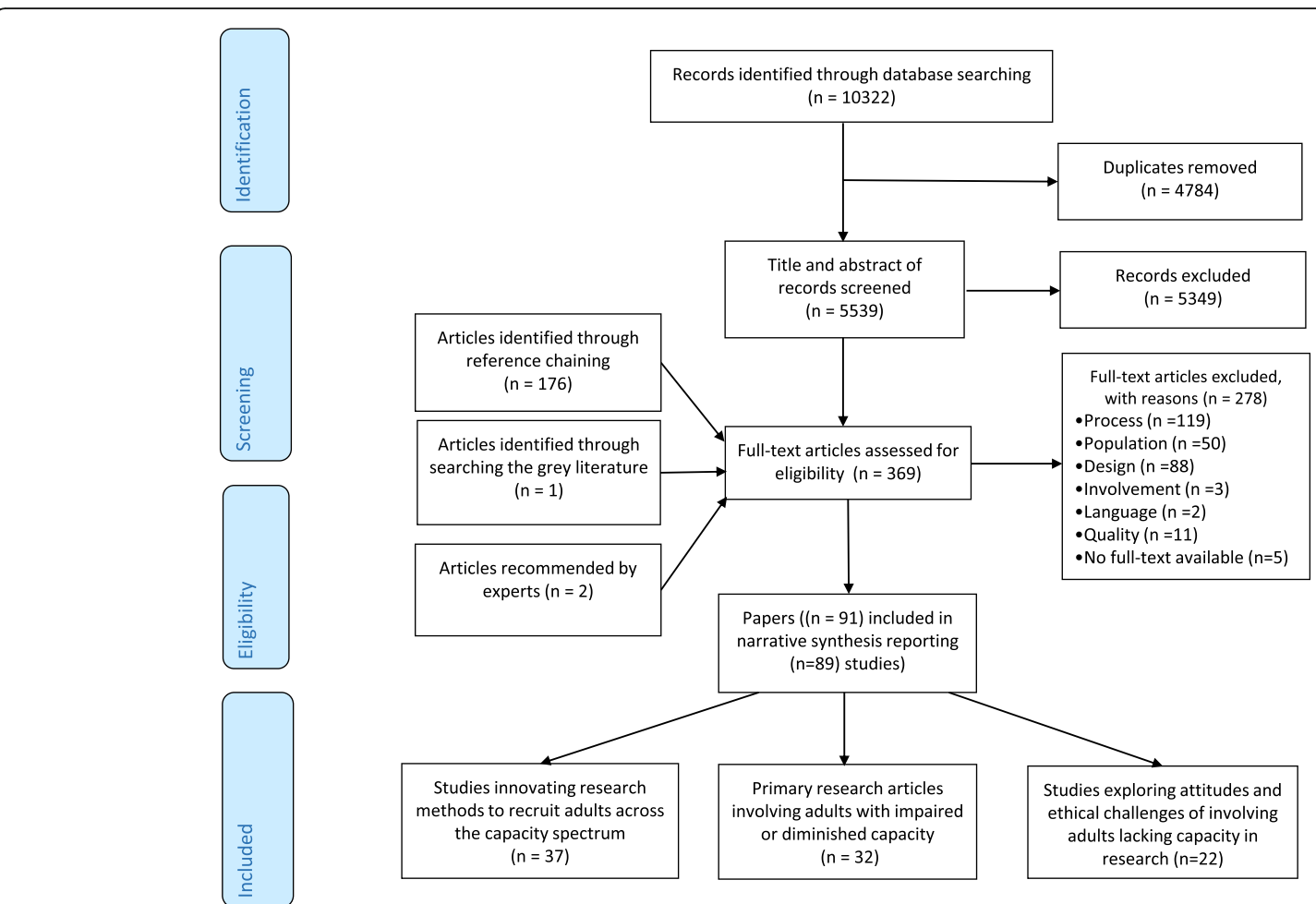

Fig. 2 PRISMA flow diagram

person from receiving and considering study information [43], and enabling understanding and recall of the information provided $[59,103]$. Moreover, in ensuring an informed consent rather than participation based on trust in the clinician and the study proposal considered a recommendation to take part [103]. A study observing consent process for people with dementia $(n=46)$ and their surrogate decision-maker (e.g. spouse) across six trials (drug and non-drug) showed approaches to facilitating the consent process and wide variation [60]. Importantly, the consent process involved the person with dementia and their surrogate, with the surrogate playing a major role in facilitating the consent process. Use of a dual consent was common, but assent from the person was often implicitly implied not explicitly asked. The study revealed wide variability in the conduct of the consent process with higher risk drug trials generally more comprehensive in giving study information and assessing understanding compared to the non-drug trials. The authors make recommendations on using a dual consent

Table 2 Population of interest categorised by the study focus

\begin{tabular}{|c|c|c|c|c|}
\hline \multirow{2}{*}{$\begin{array}{l}\text { Patient population of } \\
\text { interest }\end{array}$} & \multicolumn{4}{|l|}{ Study focus on processes of consent } \\
\hline & Innovating research methods $(n=37)$ & $\begin{array}{l}\text { Applying research methods } \\
(n=30)\end{array}$ & $\begin{array}{l}\text { Attitudes and ethical considerations } \\
(n=22)\end{array}$ & $\begin{array}{l}\text { Total studies } \\
(n=89)^{*}\end{array}$ \\
\hline Palliative care/cancer & $6[43-48]$ & $8[49-56]$ & $2[57,58]$ & 16 \\
\hline Dementia & $13[59-71]$ & $10[40,42,72-79]$ & $1[80]$ & 24 \\
\hline Geriatric care & $2[81,82]$ & $3[83-85]$ & 0 & 5 \\
\hline Parkinson's disease & $1[86]$ & 0 & 0 & 1 \\
\hline Cerebral ischaemic stroke & $2[87,88]$ & 0 & $1[89]$ & 3 \\
\hline Mental health & $6[90-95]$ & $2[96,97]$ & $1[98]$ & 9 \\
\hline Delirium & $2[99,100]$ & $2[101,102]$ & 0 & 4 \\
\hline Intensive care & 5 [103-107] & $5[108-112]$ & $4[113-116]$ & 14 \\
\hline General population & 0 & 0 & 13 [117-129] & 13 \\
\hline
\end{tabular}

*89 studies are reported from 91 publications (includes two study protocols [40,42] reported with the main study papers [39, 41] 


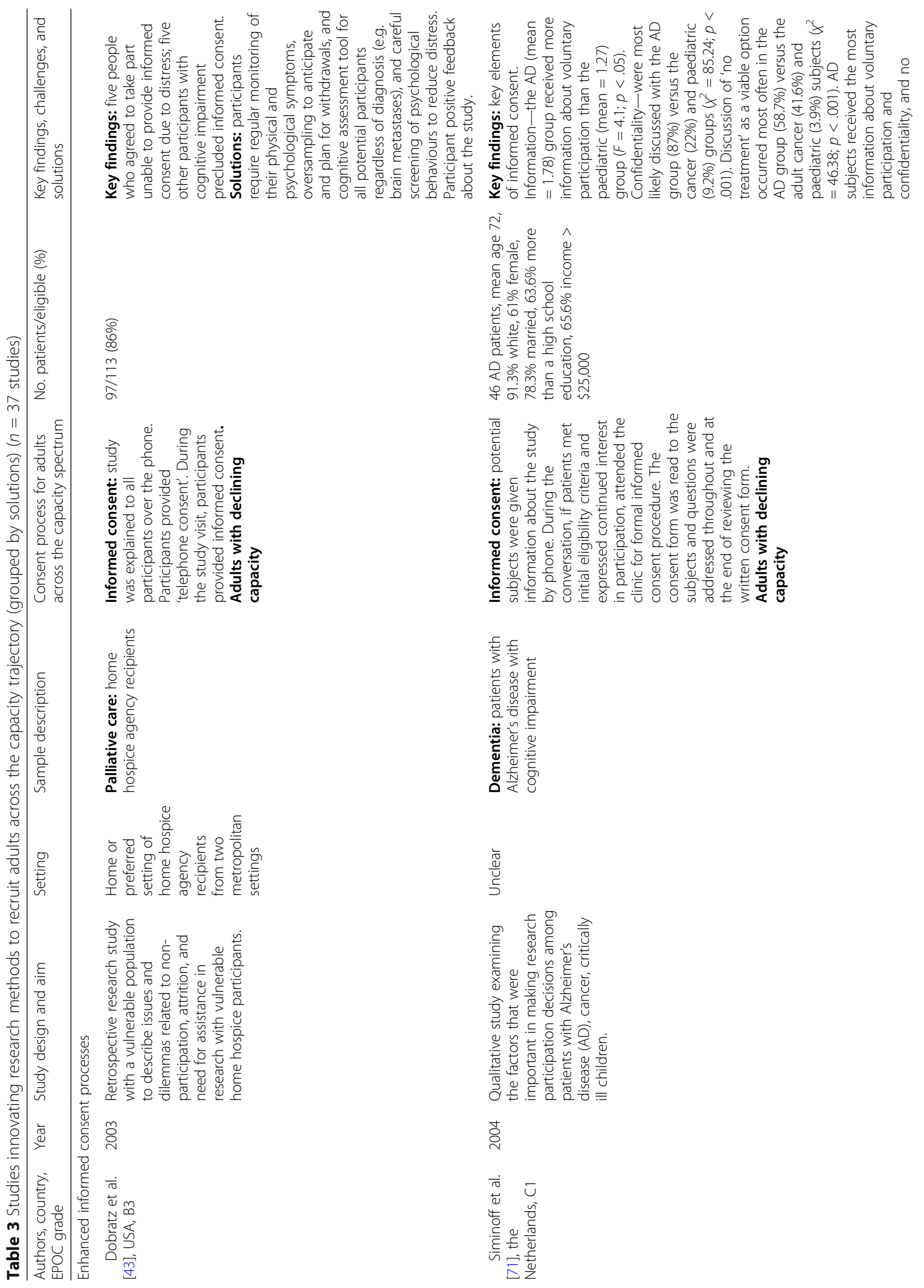




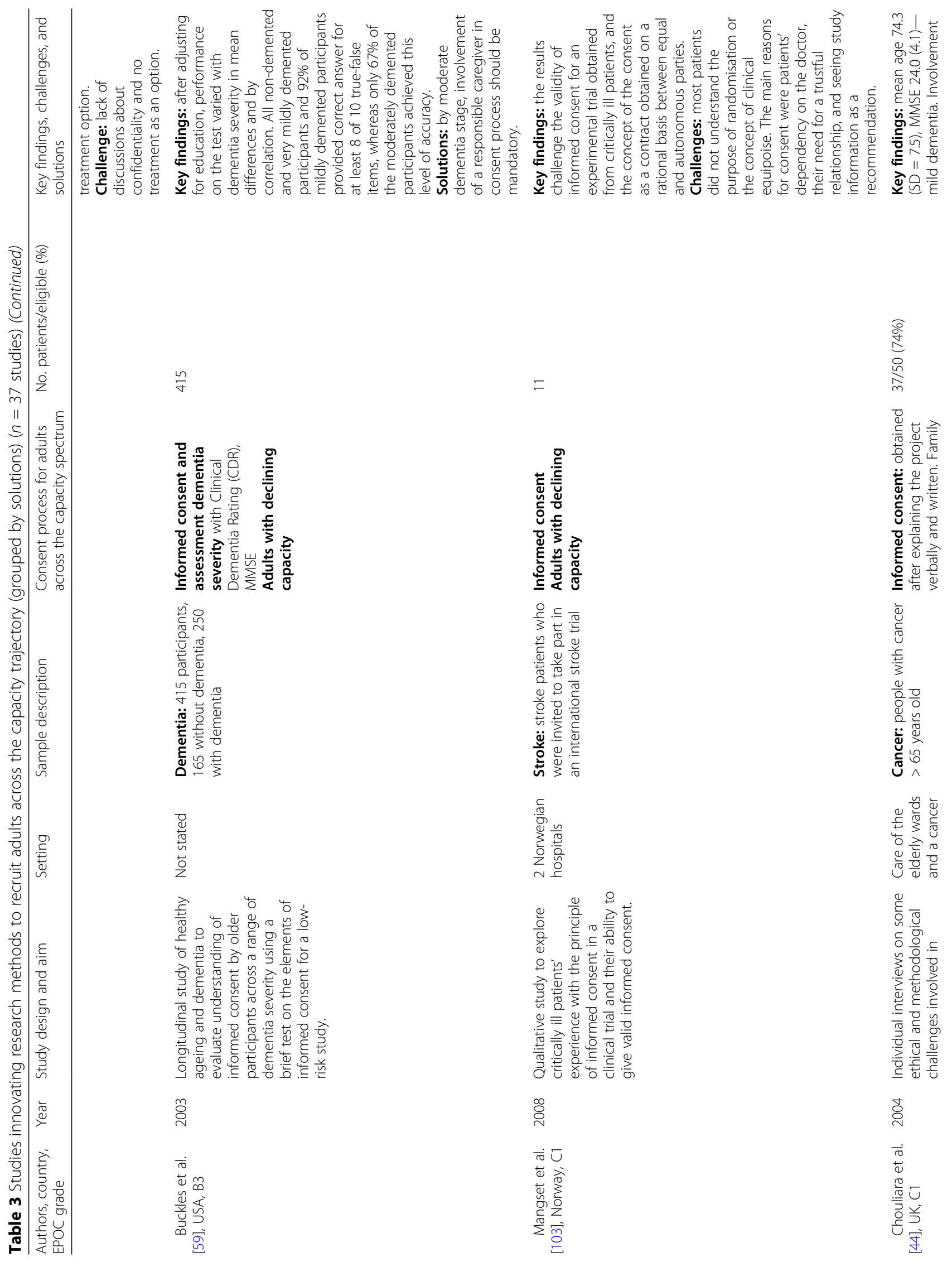



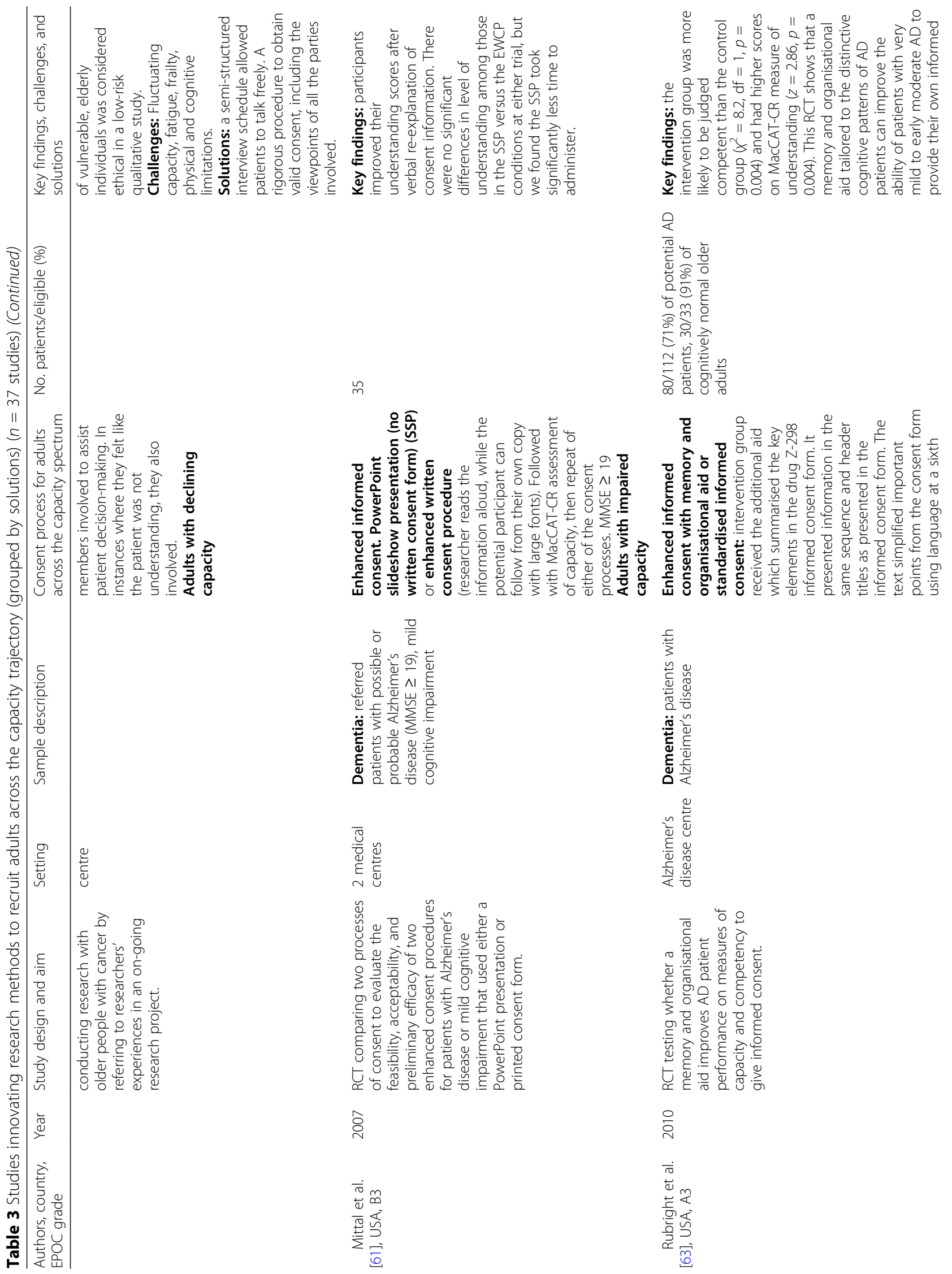

$\stackrel{m}{m}$
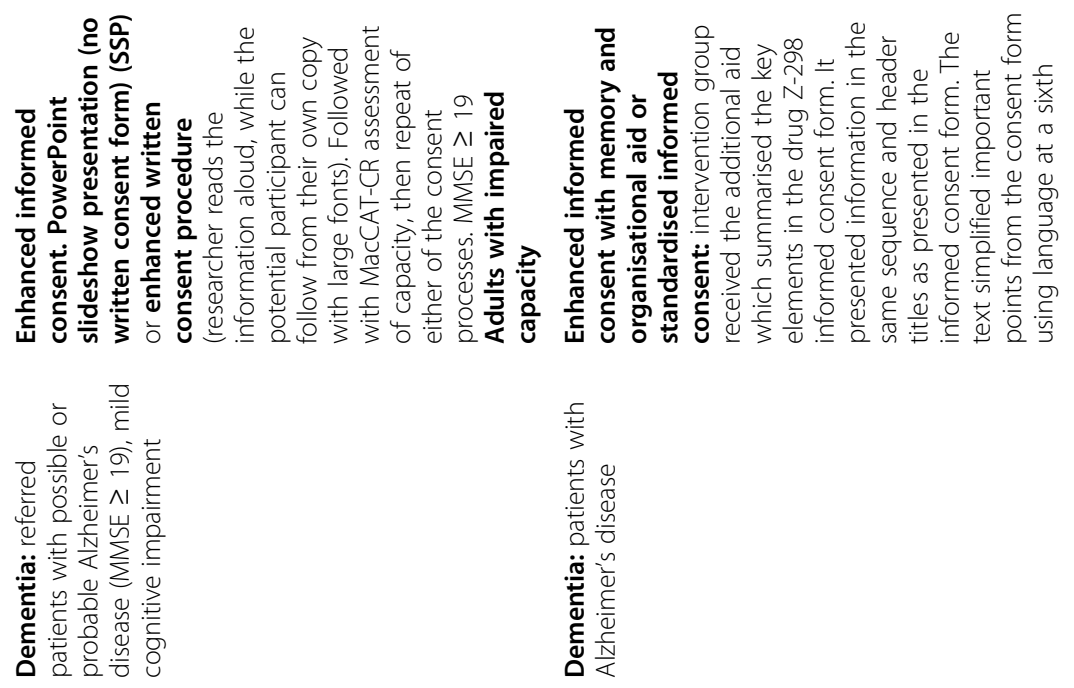

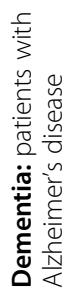

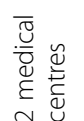

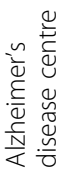

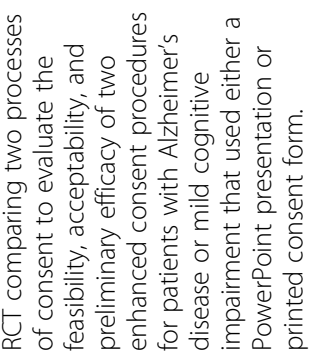

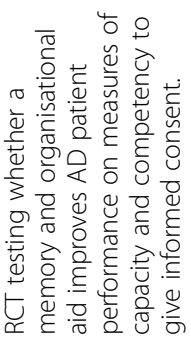

\&े

호

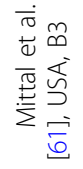

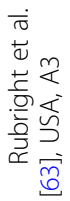




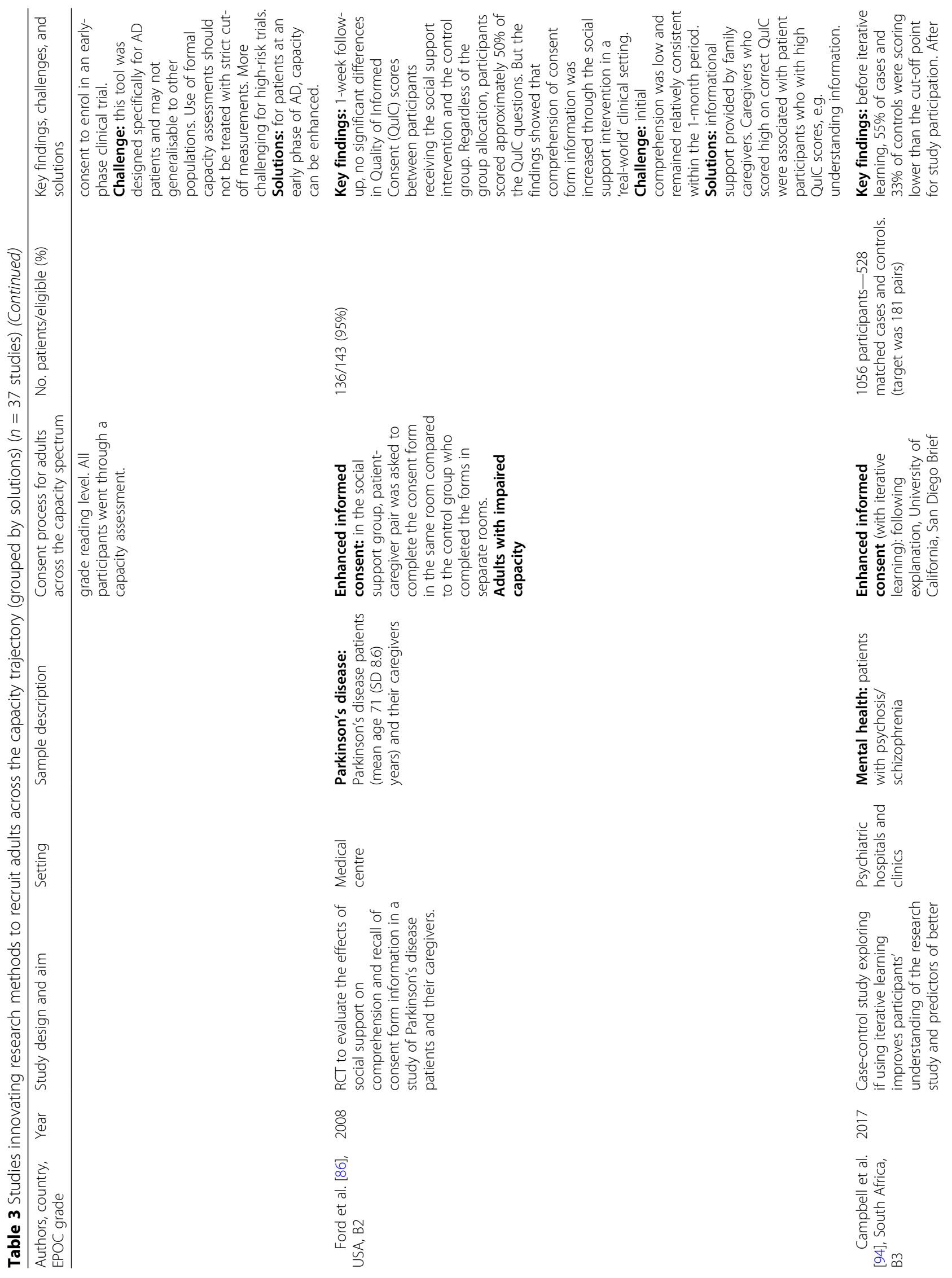




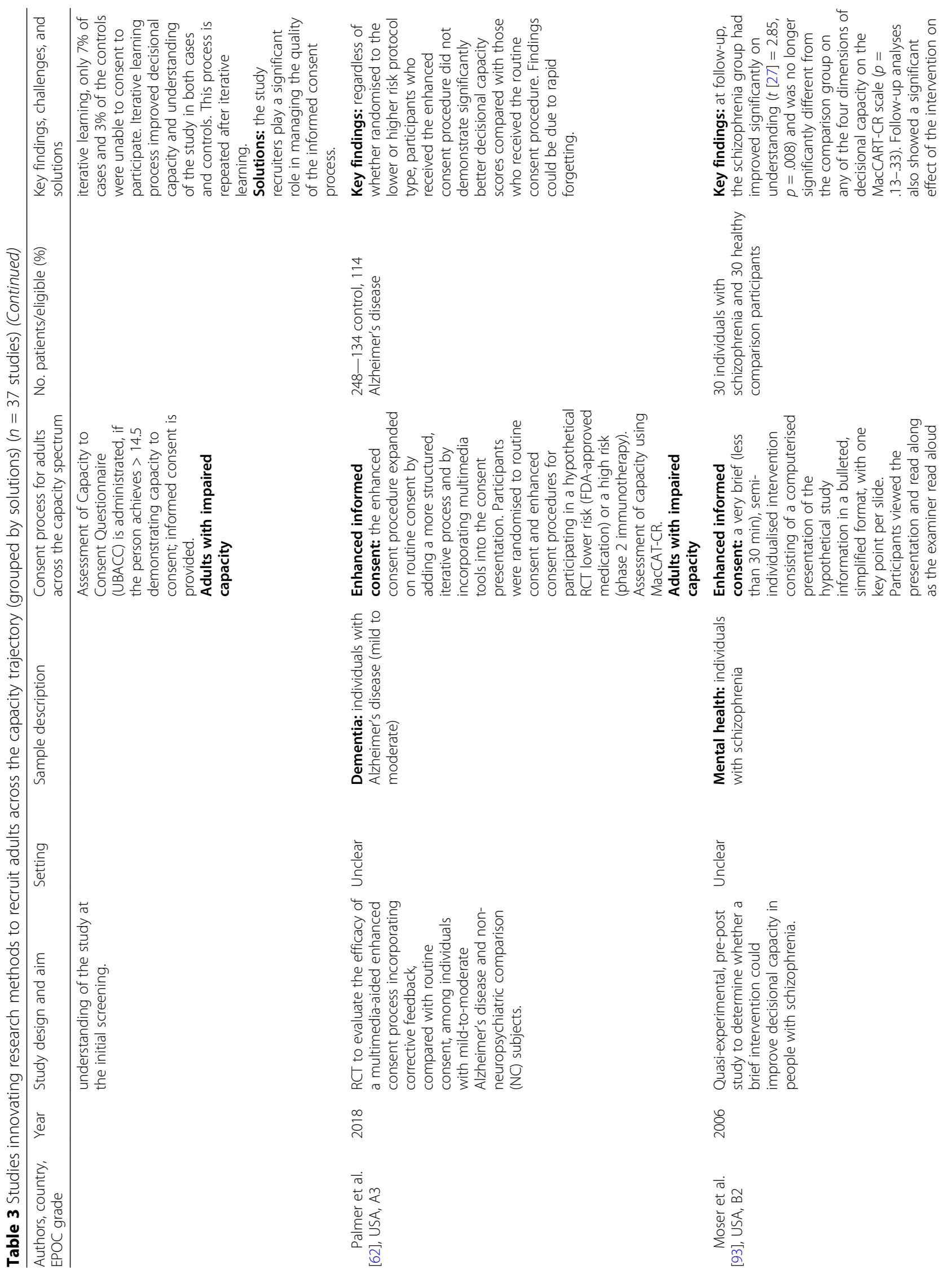




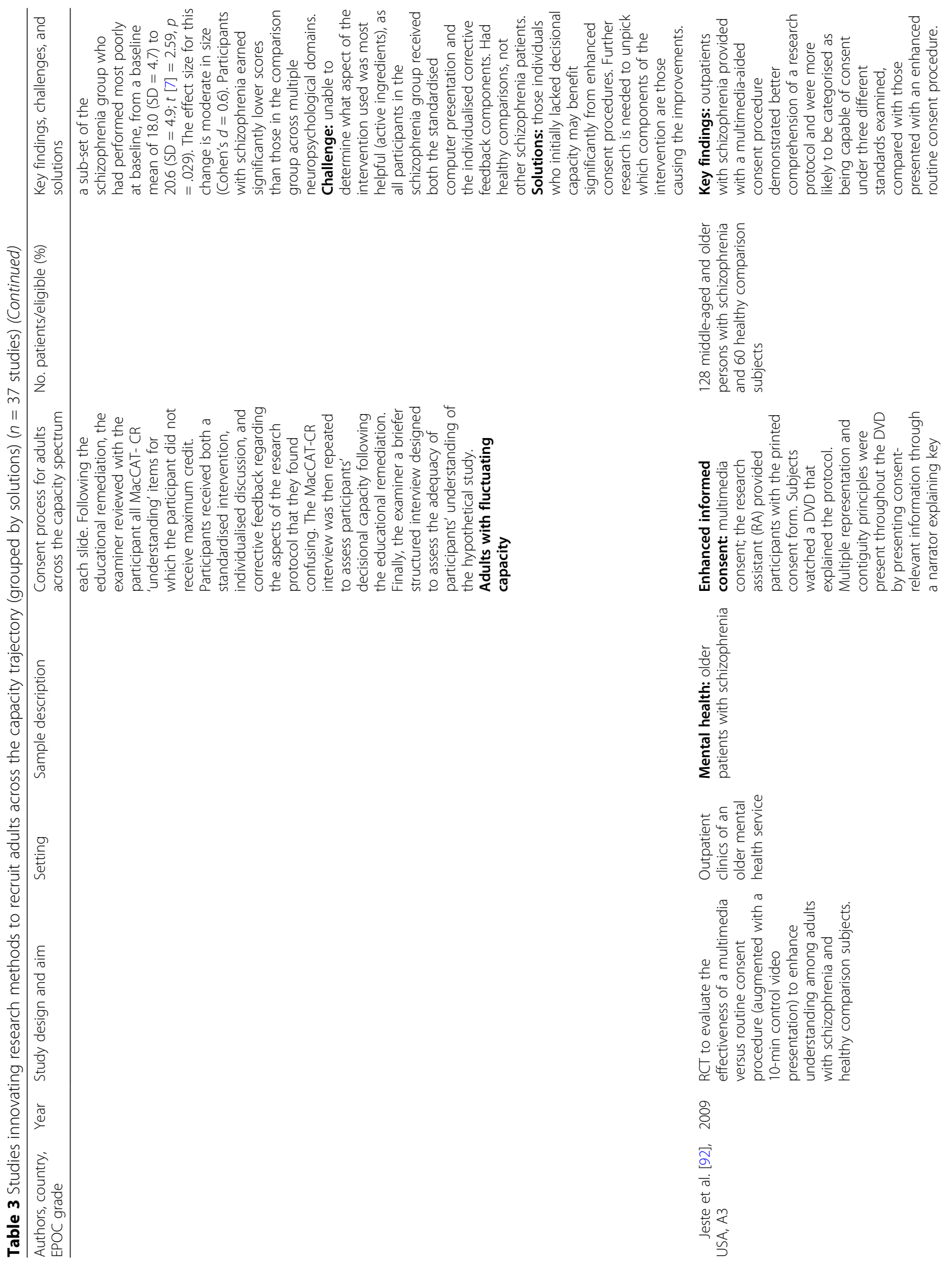




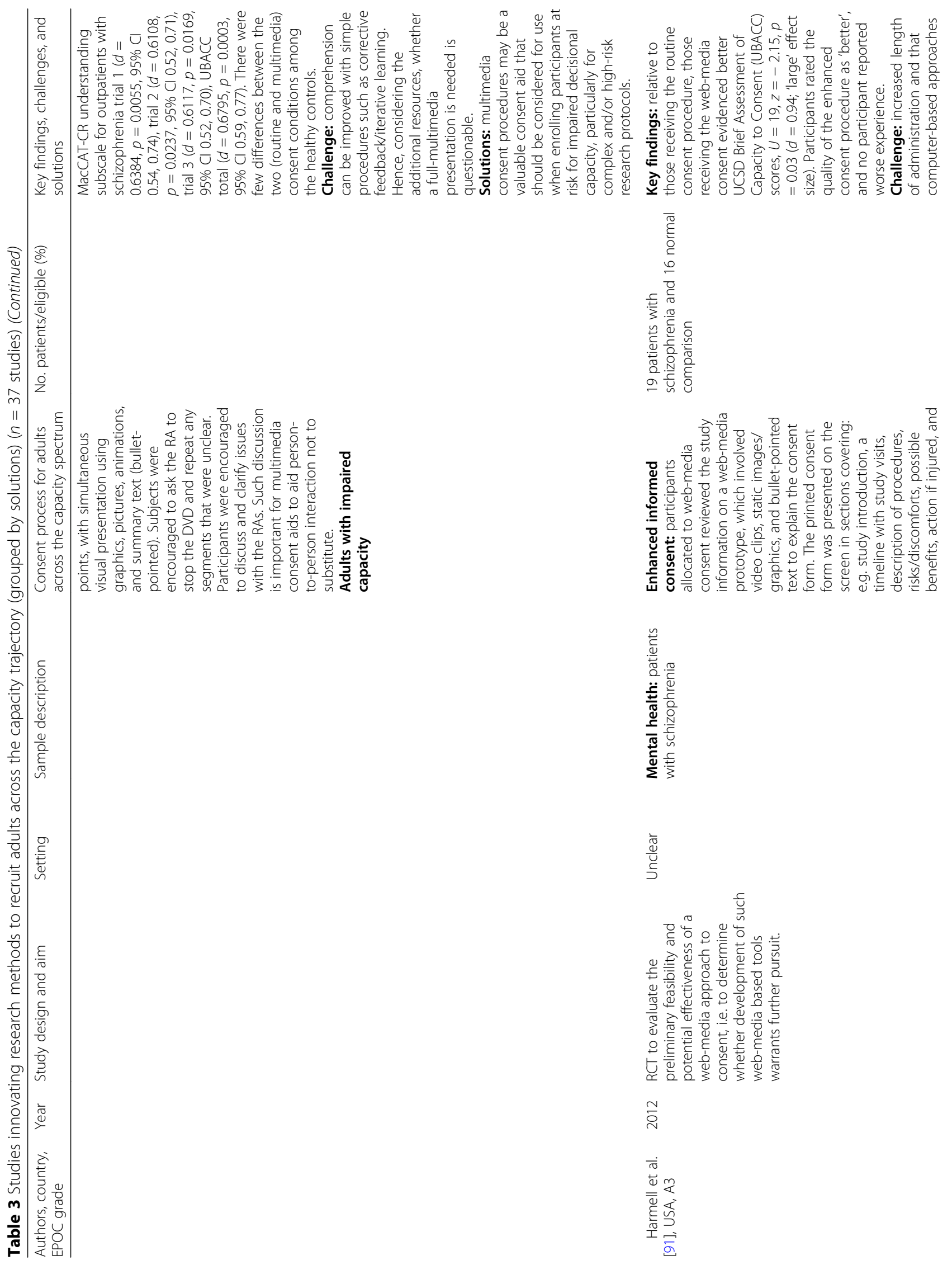




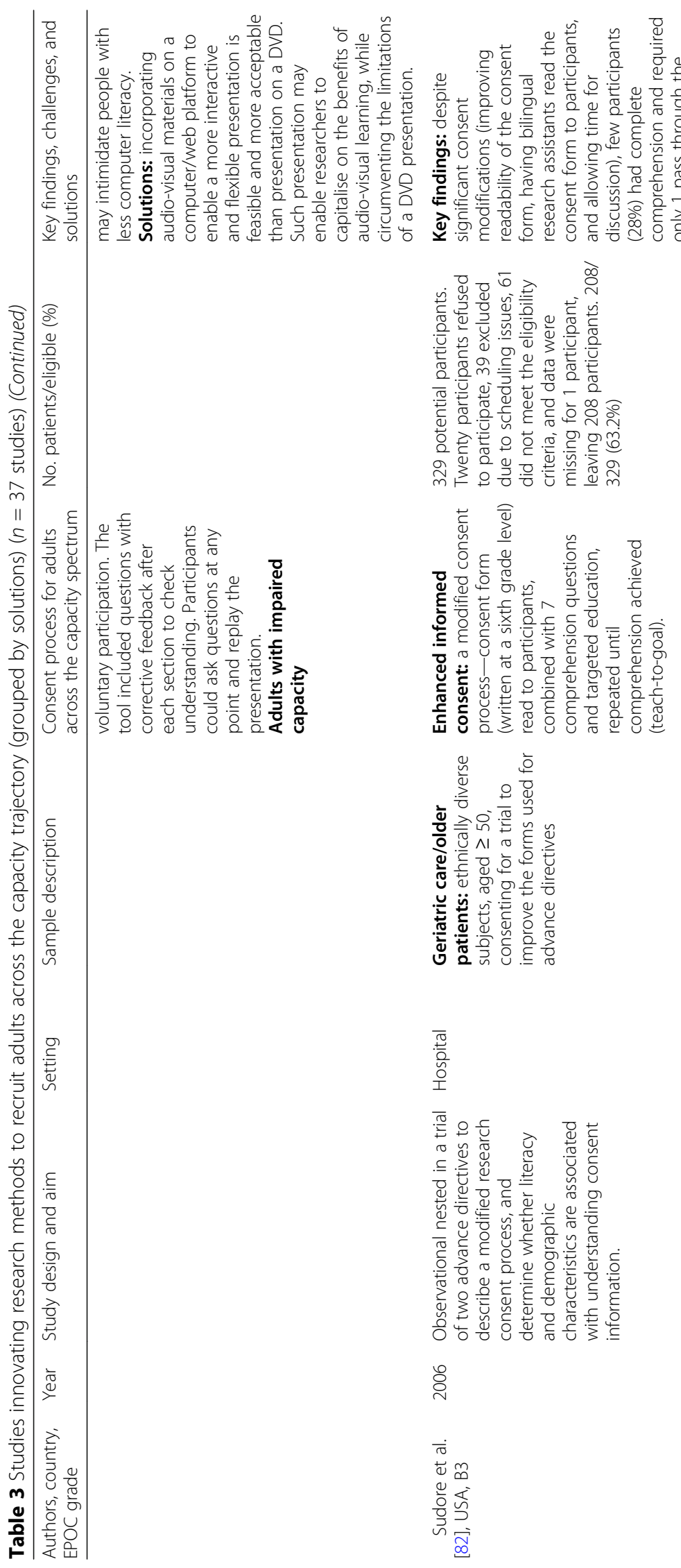




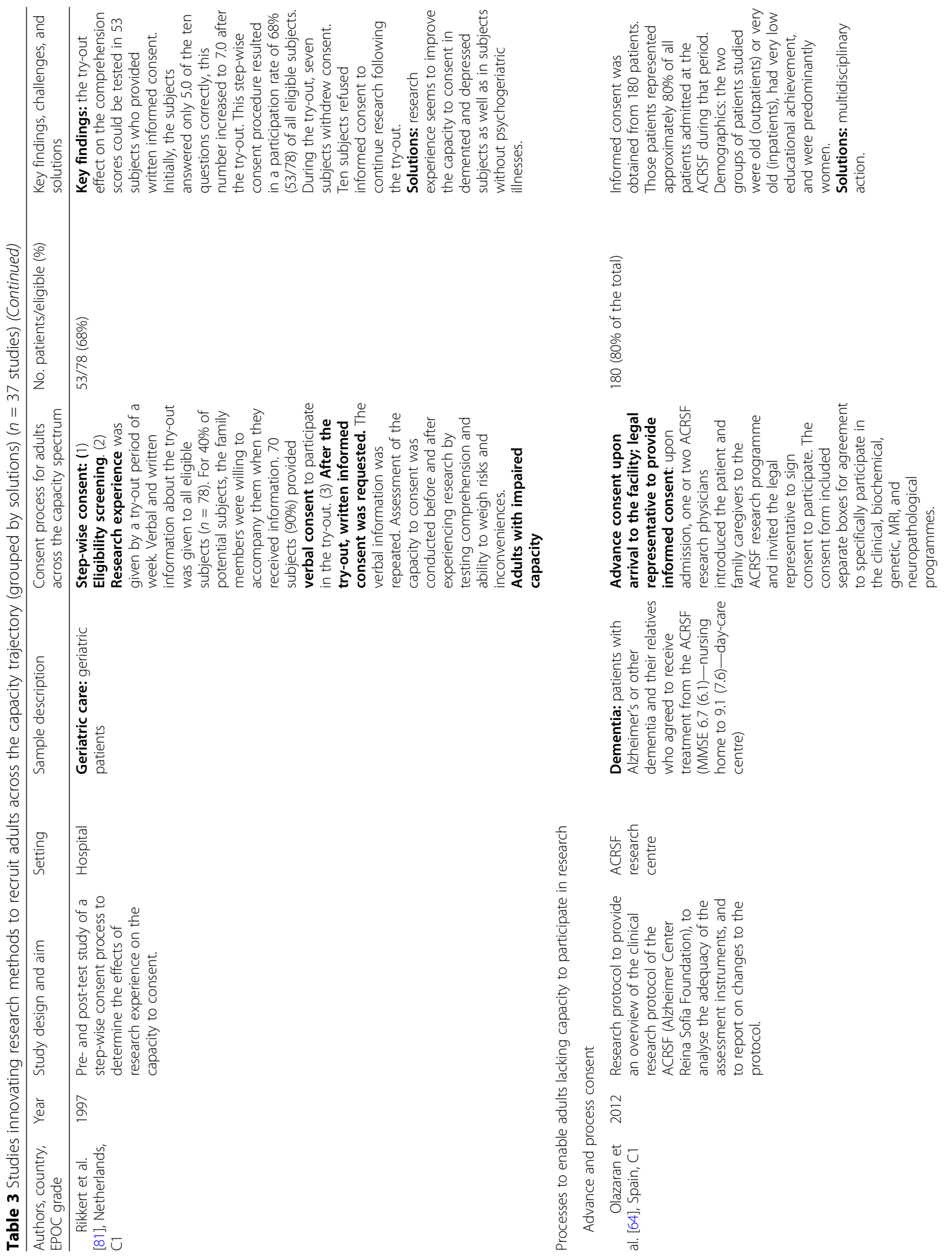




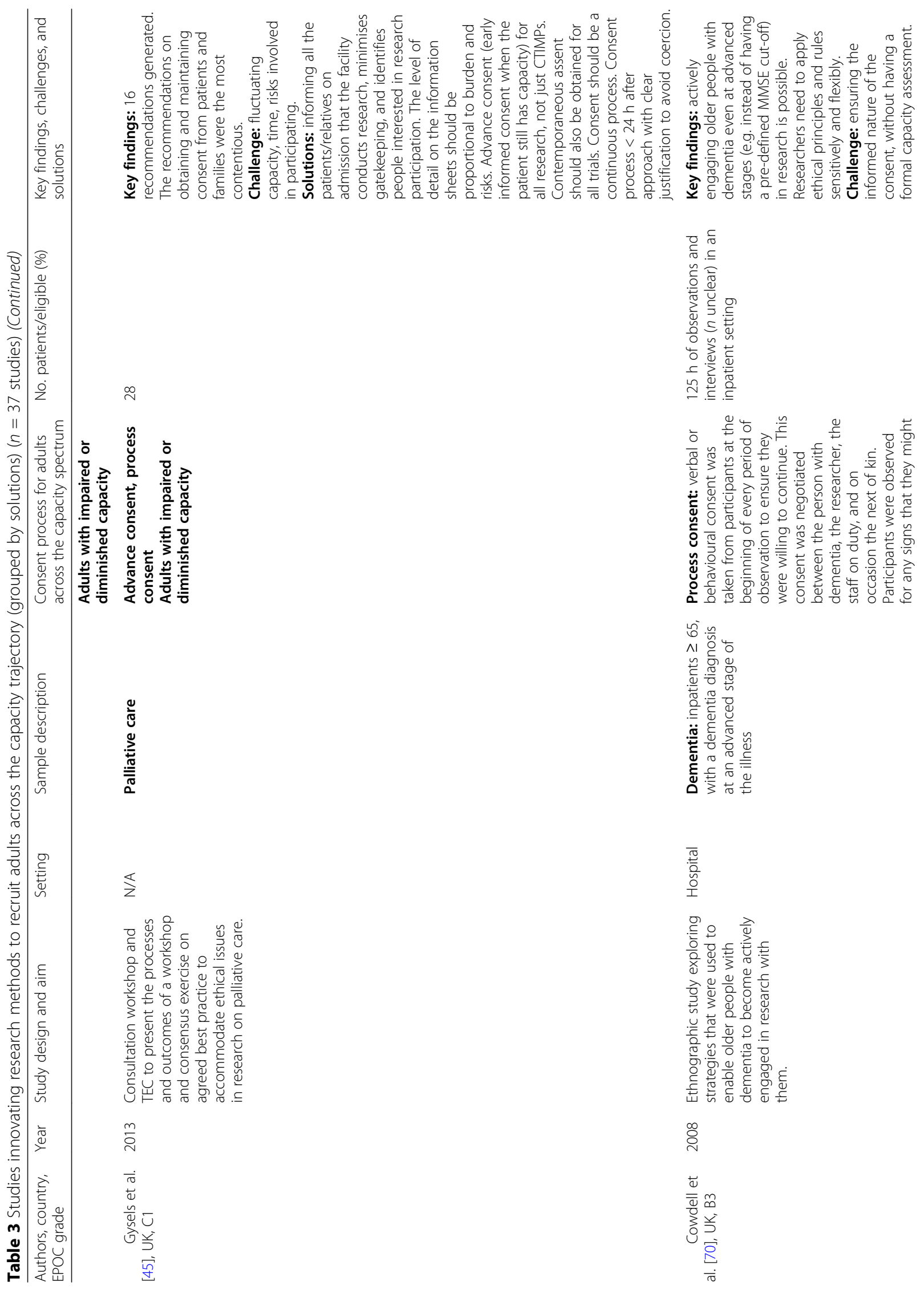




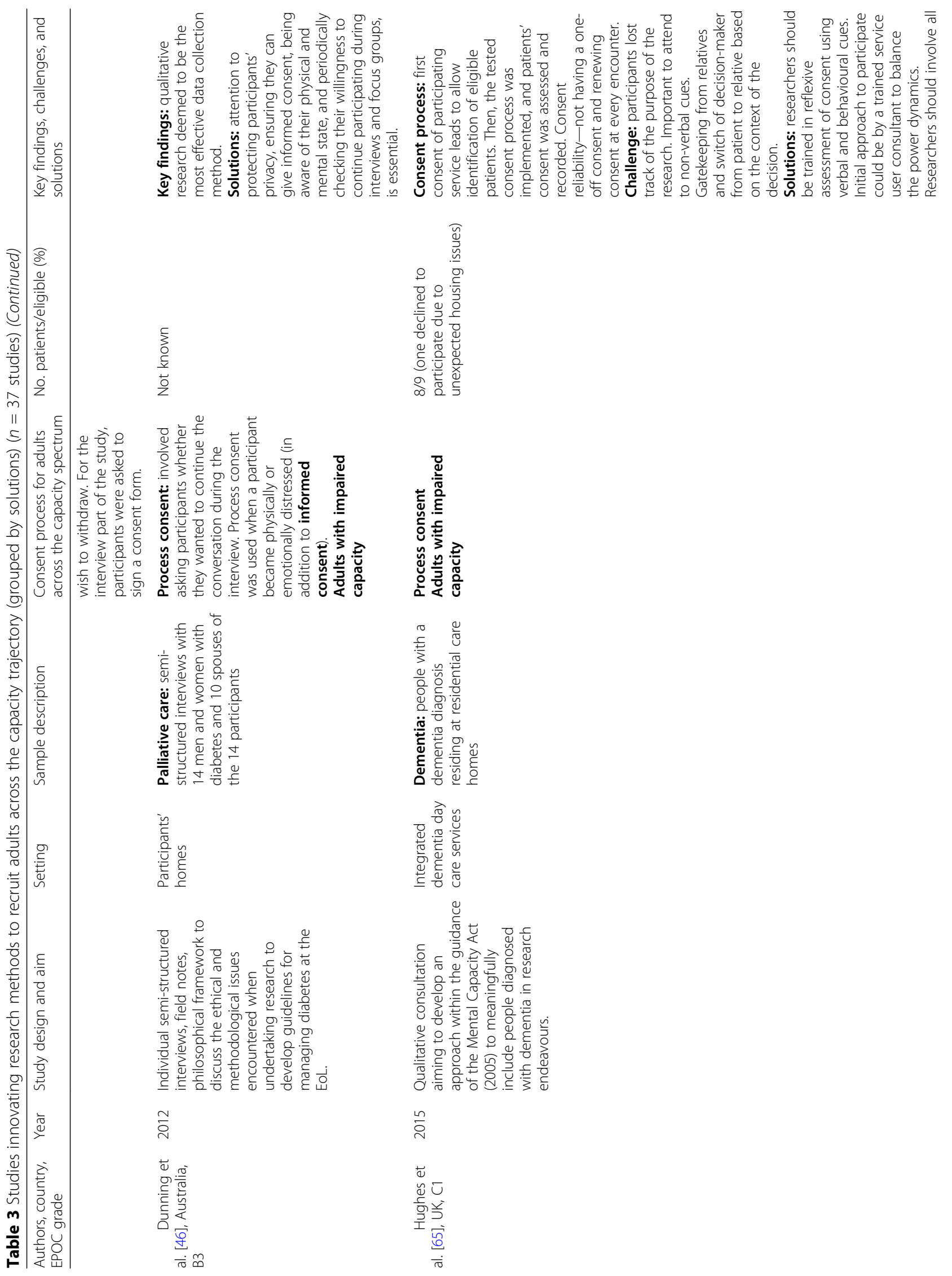




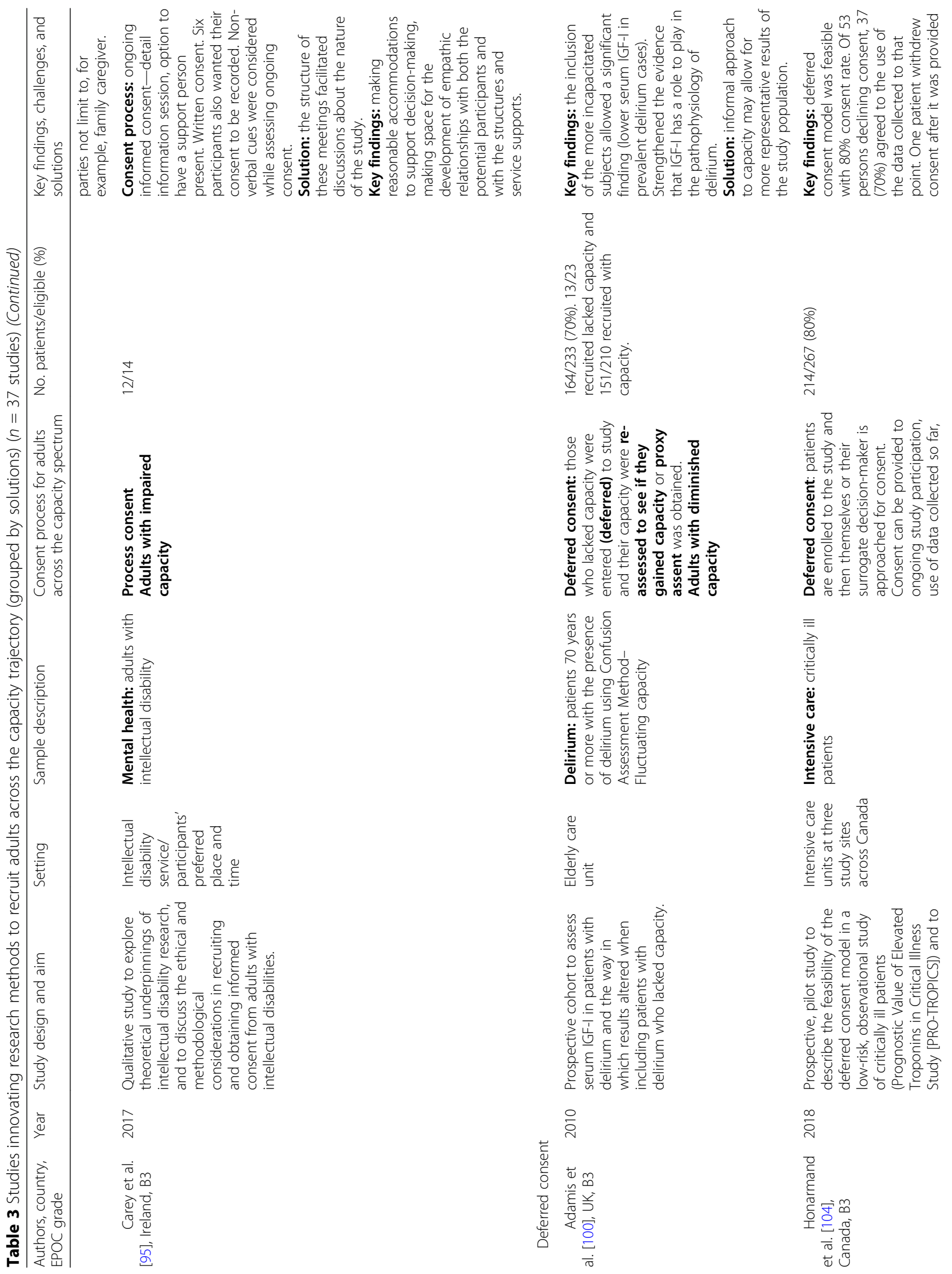




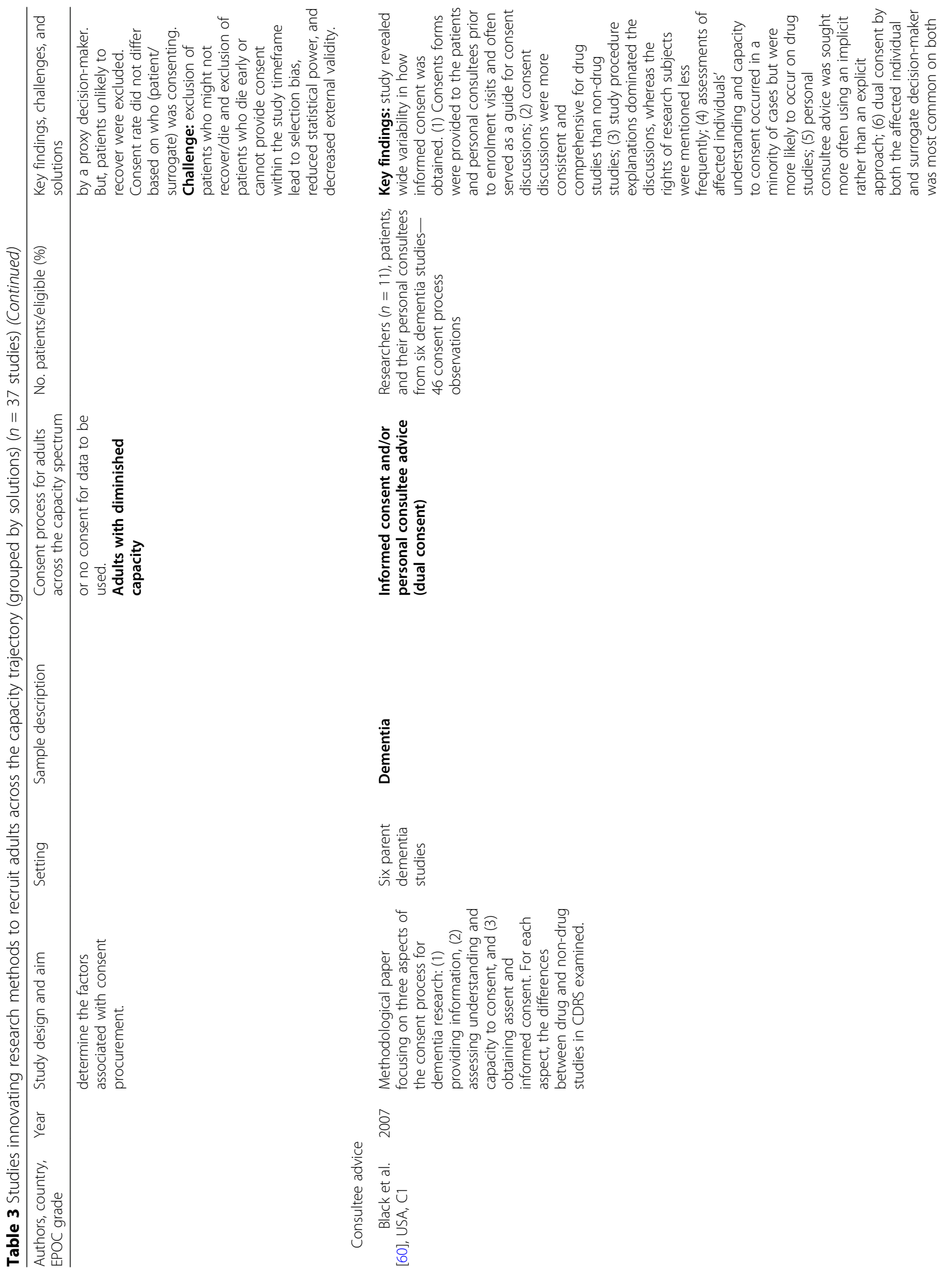




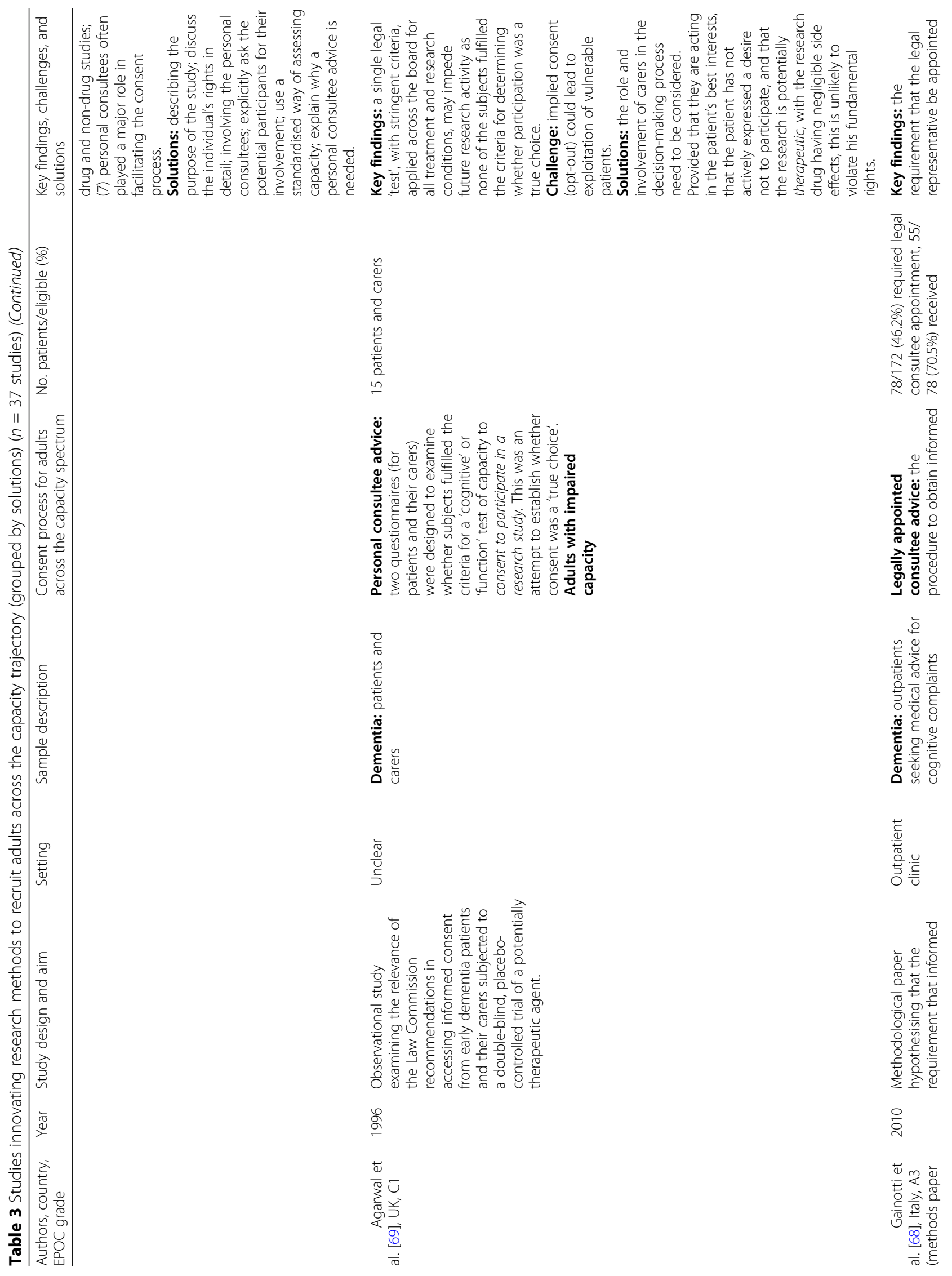




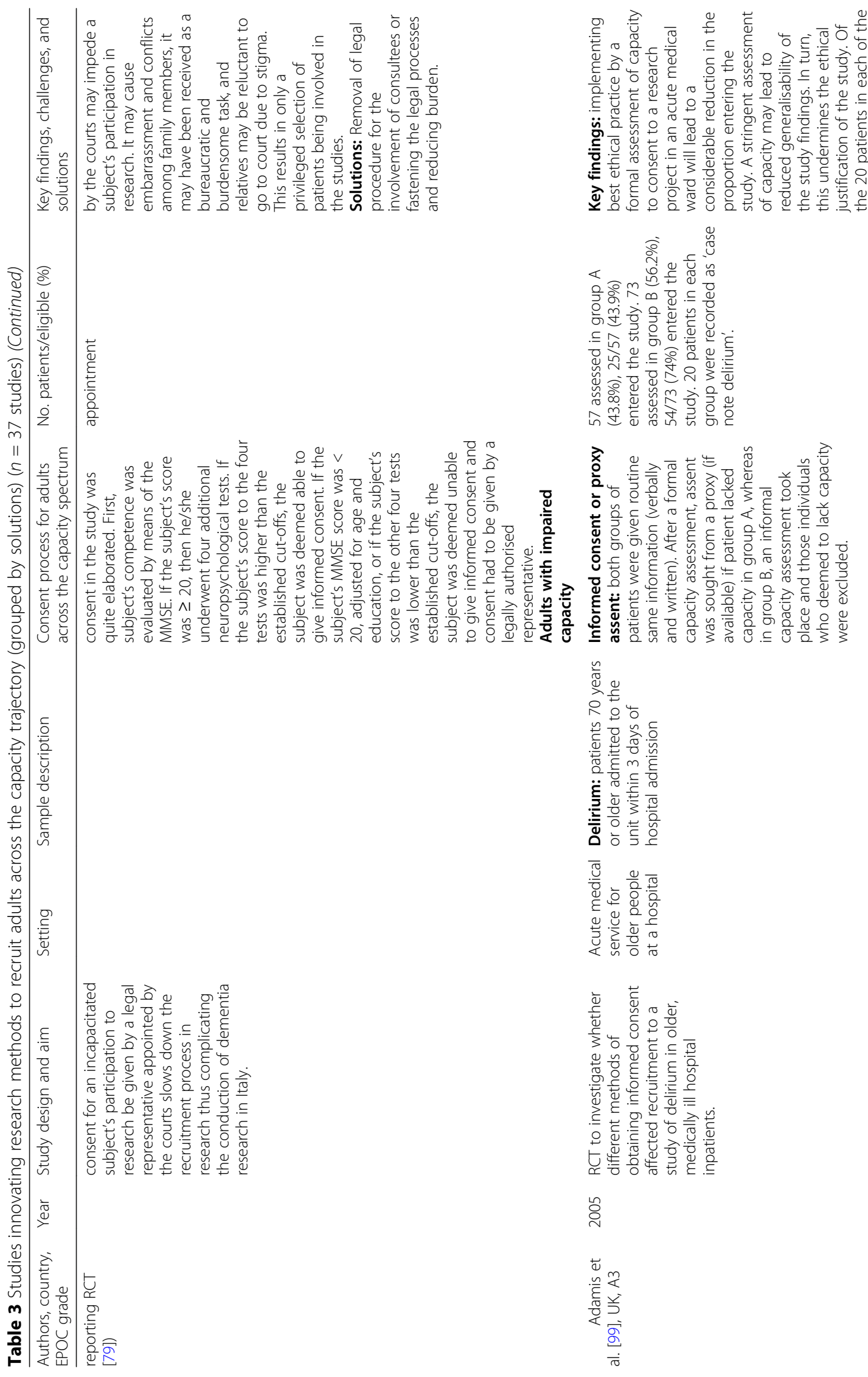




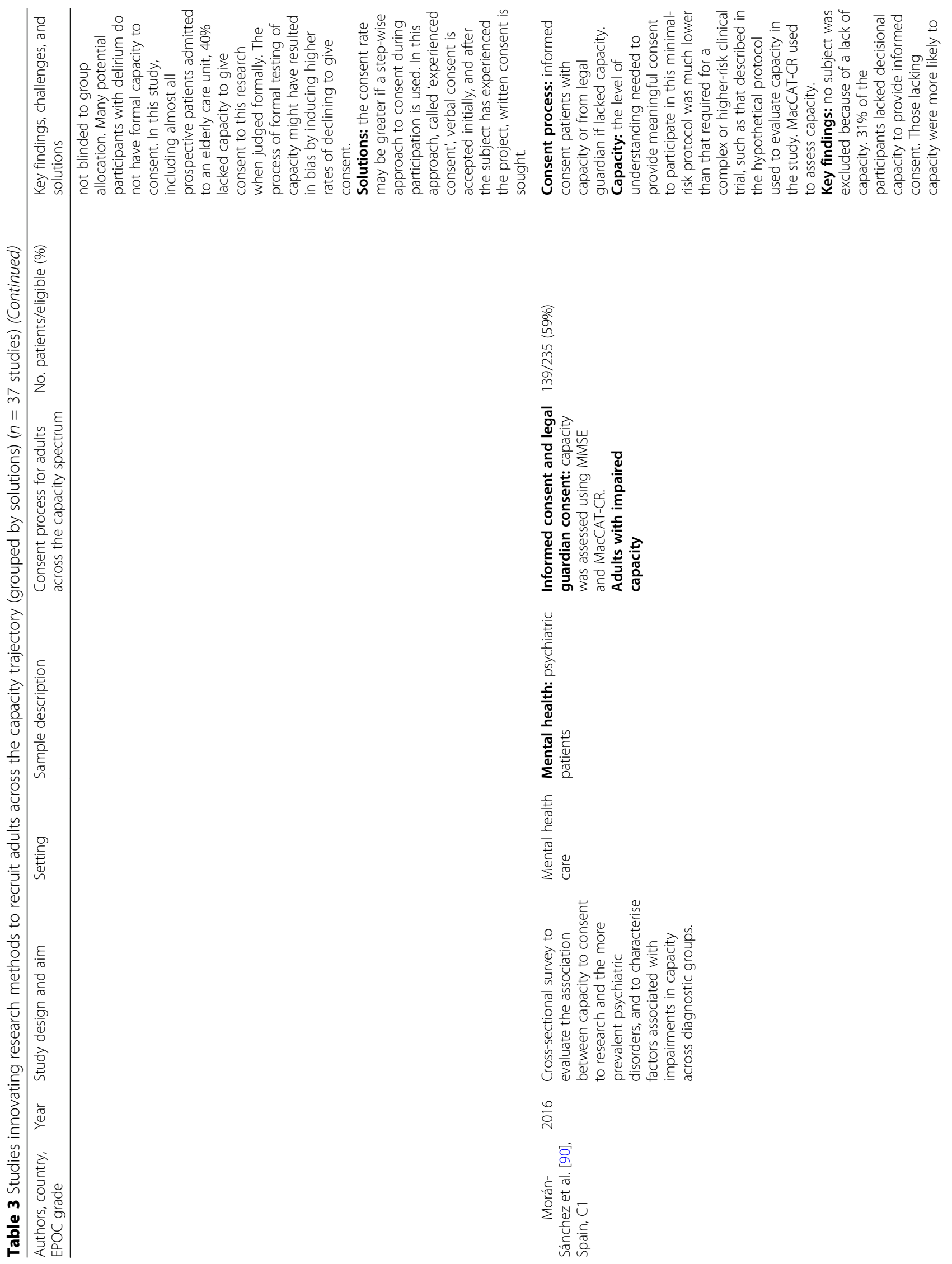




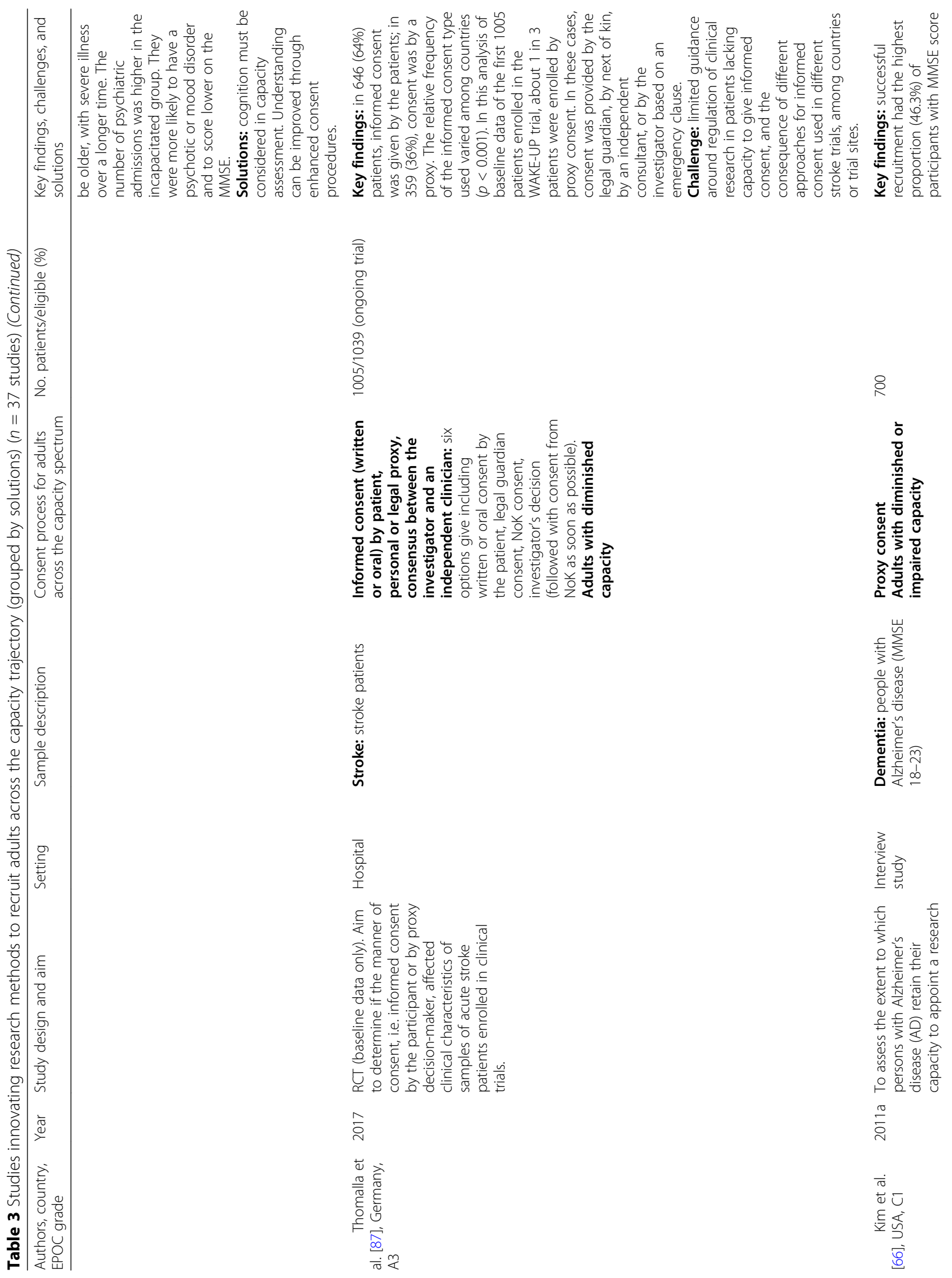




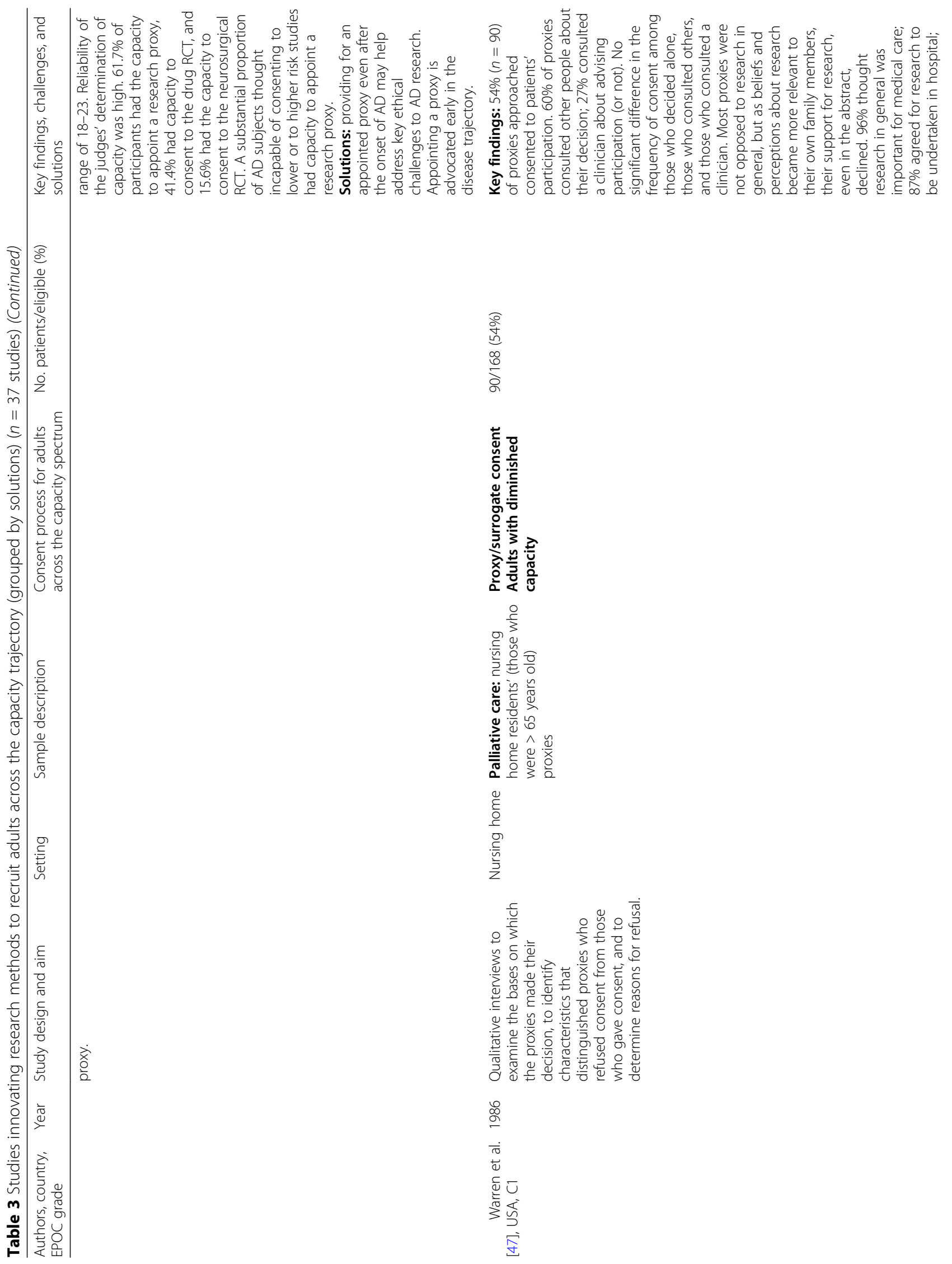




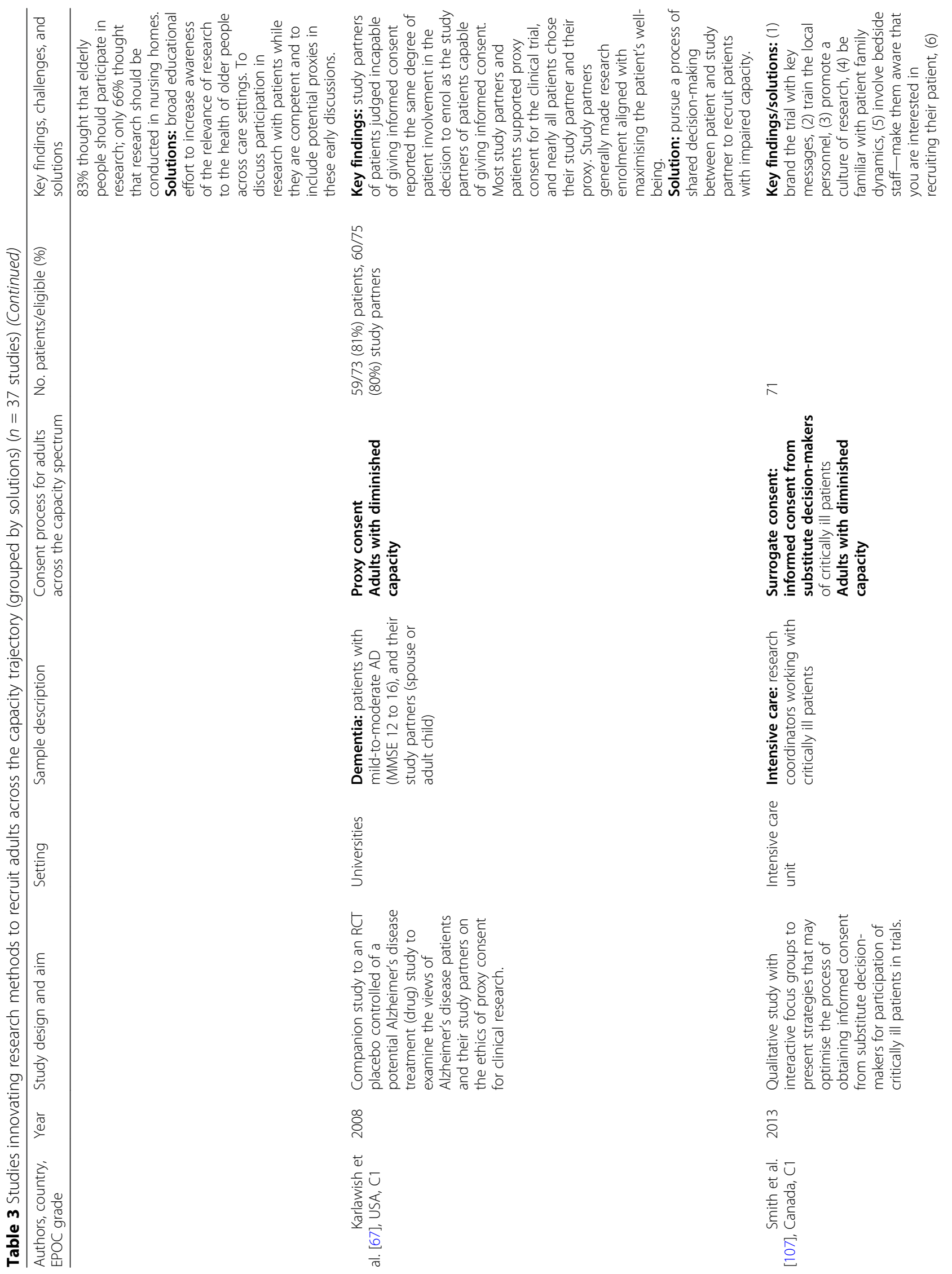




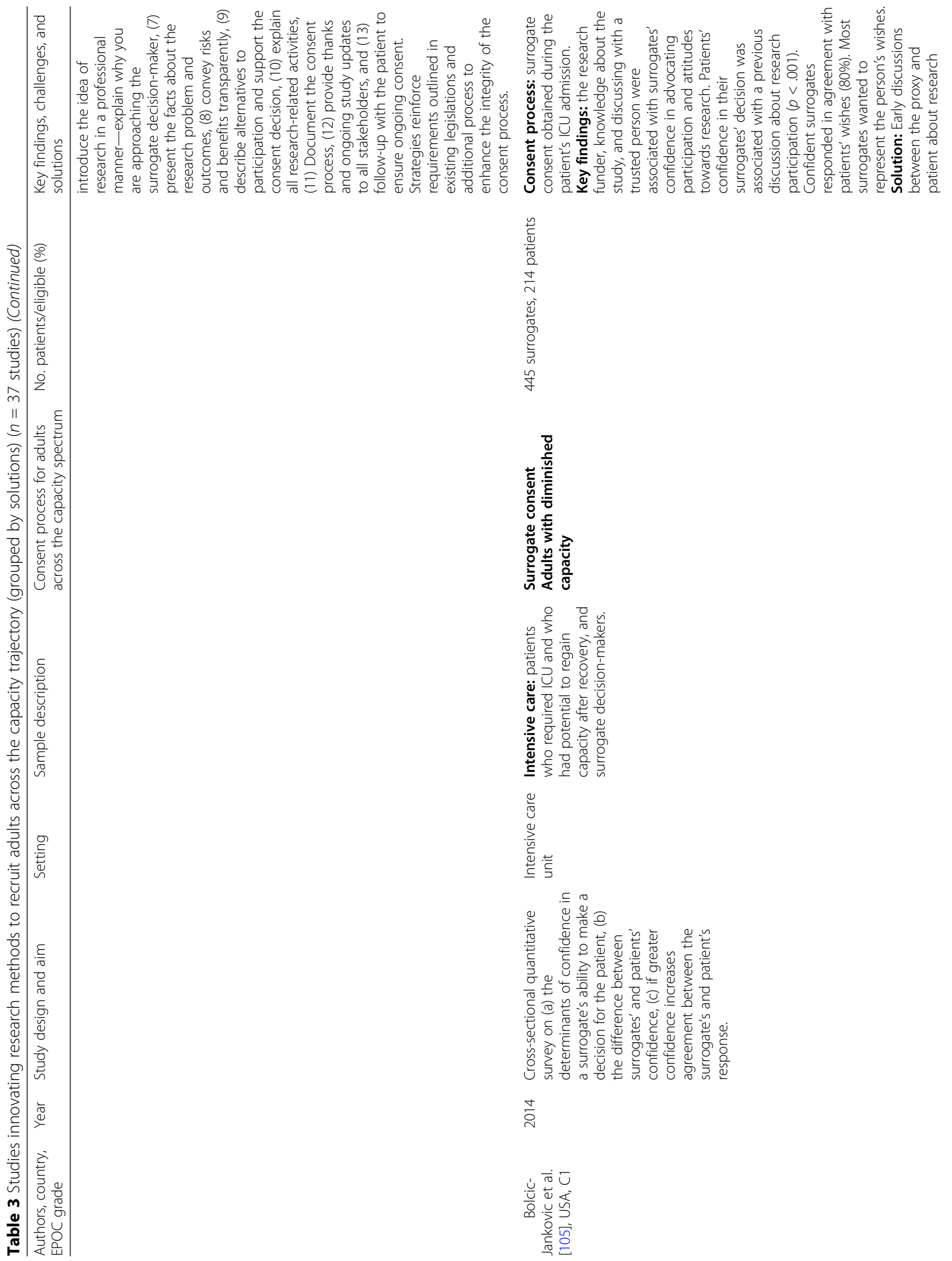




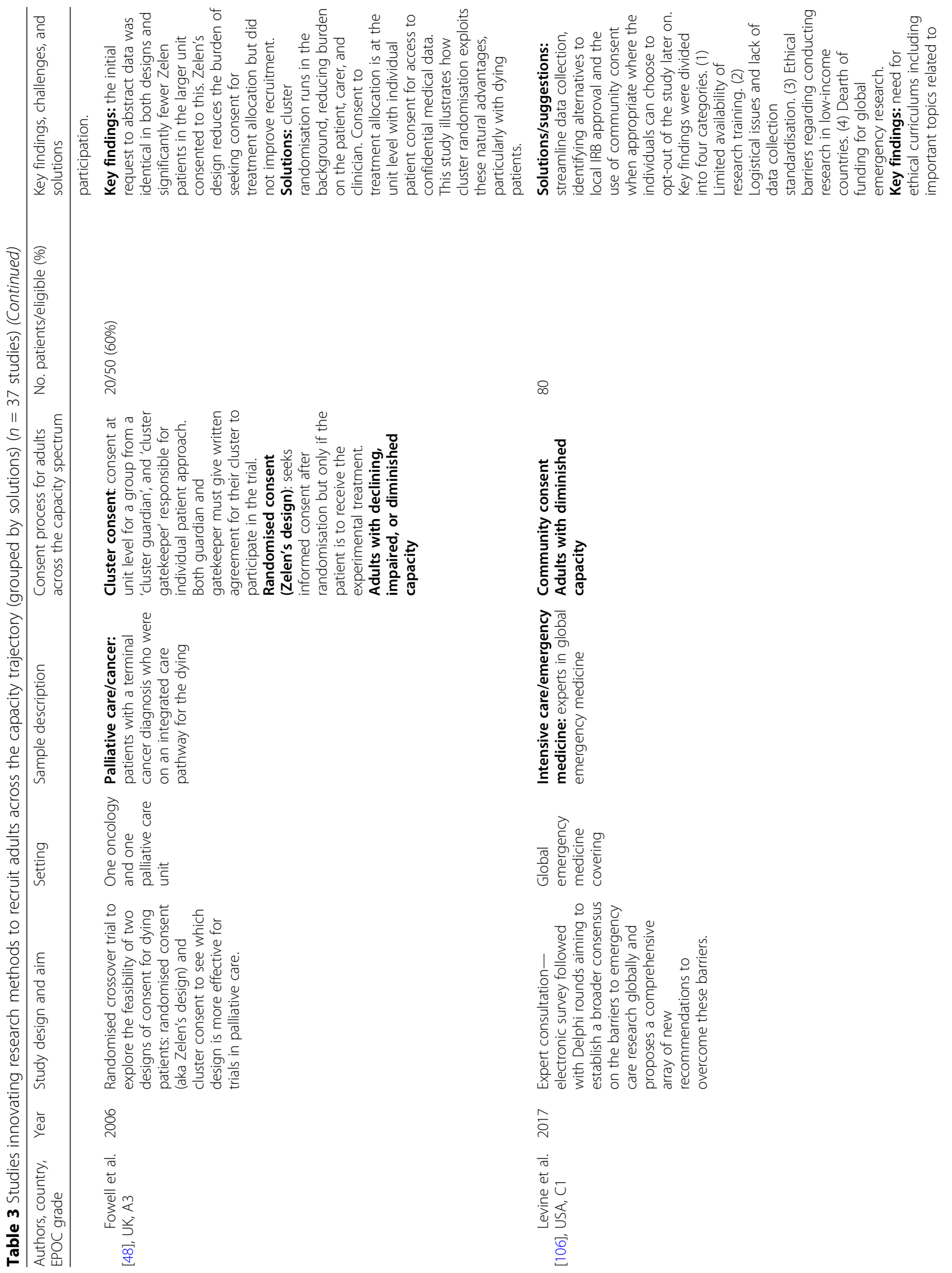




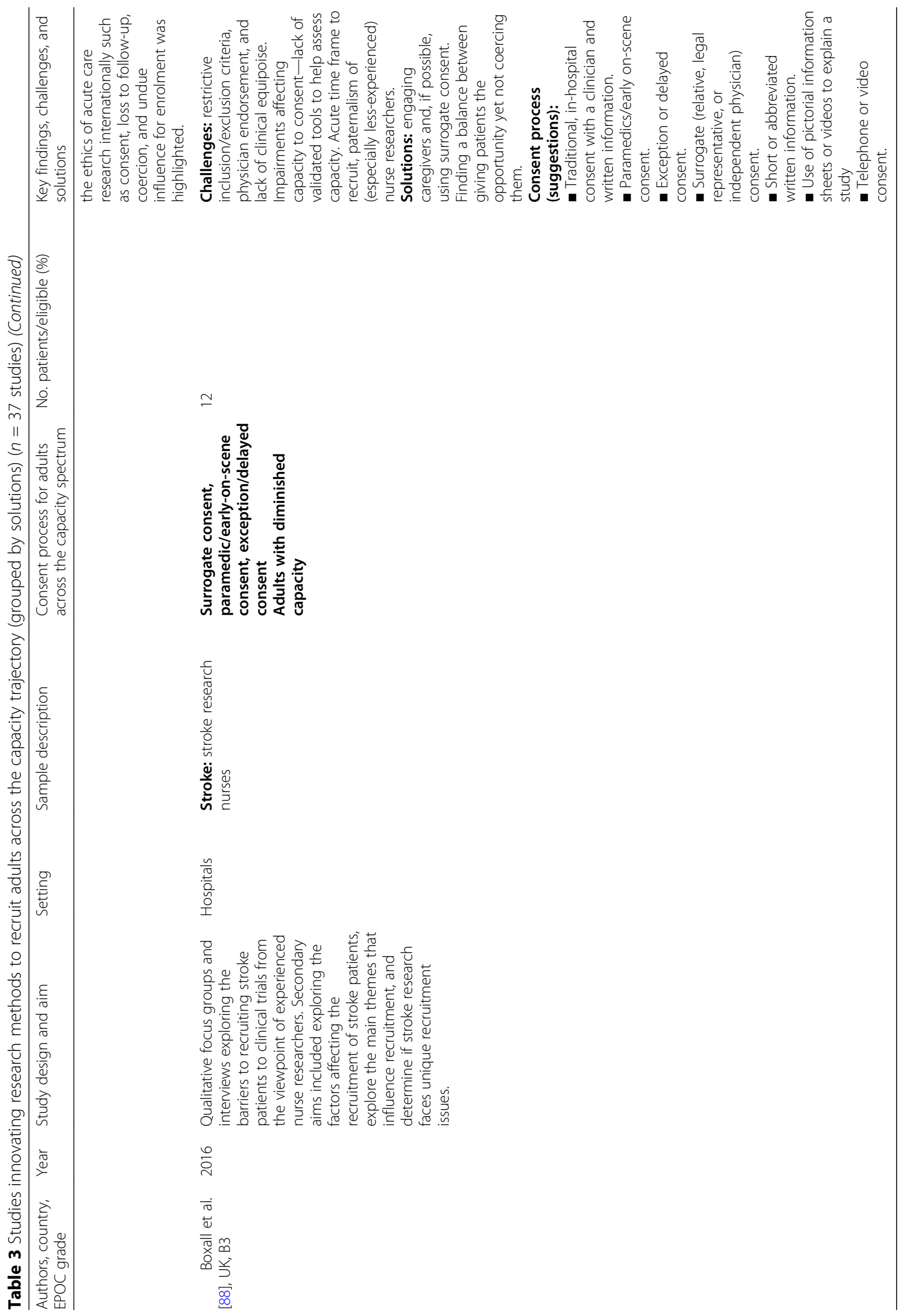


process and standardising the information provided to encompass both study procedures and participants' rights to, for example, withdrawal.

Successful enhanced informed consent processes often included providing information in more than one format (generally verbal and written) [61], improving understanding by using novel techniques (e.g. simplified storybook, video) [91-93], and tailoring the process of study approach to a person's psychological and physical health status [43]. In studies involving patients with schizophrenia $[91,93,94]$ or dementia $[62,63]$, using a combination of multimedia techniques and flexibility to repeat aspects of the study information significantly improved individuals' understanding about the study, and to give an informed consent. Studies using enhanced informed consent processes reported generally high consent rates for eligible participants ranging from $68.0 \%(n=53 / 78)$ in an elderly patient population [81] (mean age 80.1 years) to $95.1 \%(n=136 / 143)$ in patients with Parkinson's disease (mean age 71 years) [86]. The addition of social support from family members enhanced older individuals' decision-making capacity. For example, a study with hospitalised older cancer patients (MMSE mean 24.0, SD 4.1) achieved 74\% (37/50) recruitment rate by formal involvement of family members including presence during the consent process, asking their views, encouraging patients to discuss with the family members, and taking their concerns into account [44]. In an RCT $(n=136)$ on the effect of social support on the consent process for patients with Parkinson's disease (mean age 71 years, SD 8.6), the presence of a family member compared to the patient alone showed effect on enhancing comprehension and recall of the study information at 1 week $(p=0.012)$ and 1 month $(p=0.040)$ [86].

However, the evidence for using multiple or iterative techniques to enhance individuals' understanding and reasoning was mixed. Variation was related to the context and the processes used. Using techniques of iterative learning by, for example, quizzing potential participants' understanding of the study and enhancing how information was provided [86, 92] enabled study engagement for individuals with lower levels of health literacy from, for example, lower socioeconomic backgrounds [82] or low- to middle-income countries (LMICs) [94]. However, findings were mixed for studies recruiting adults with dementia. RCTs showed enhanced understanding $(F[1,29]=7.17, p=0.012)$ using a PowerPoint presentation, combined with verbal consent and verbal re-explanation for participants with mild Alzheimer's disease (MMSE $\geq 19) \quad(n=53)$ [61], and using a simplified memory and organisational aid to improve understanding $\left(\chi^{2}=8.2, \mathrm{df}=1, p=0.004\right)(n=110)$ [63]. Conversely, a RCT $(n=114)$ using multimedia and iterative learning for participants with mild to moderate
Alzheimer's disease (MMSE 20.9 (SD 3.9) and 22.5 (SD 3.4), by treatment arm) did not detect a significant effect on decisional capacity $\left(\chi^{2}(3)=2.63, p=0.453\right)$ [62].

\section{Processes of consent to enable recruitment of adults across the capacity spectrum}

Twenty-one studies explored research methods in the consent process for adults across the capacity spectrum. Advance consent was advocated in two studies for participants with the anticipated loss of capacity associated with a progressive condition, e.g. dementia [45, 64]. Advance consent involved early informed consent when the person had capacity, for example, on admission to a clinical facility, with consent upheld at the point of loss of capacity [64]. An area of contention was the requirement (or not) in all research studies (Clinical Trial of an Investigational Medicinal Product (CTIMP) and non-CTIMP) for the person to nominate a consultee for contemporaneous advice on continued participation in the study [45]. Best practice was conducting the advance consent process with the person and the consultee (e.g. a family member), to discuss the person's preference for continued participation should they lose capacity, and the role of the consultee on contemporaneous advice aligned to the person's wishes and the context [45, 64]. This strategy may minimise potential gatekeeping about continued participation with disease progression [45]. Process consent was proposed for studies with multiple time points that involved individuals able to understand and appraise information in the moment, but with difficulty retaining and recalling study-related information in the future, for example, individuals with mild/moderate dementia, palliative care, and mental health populations [46, 65, 95]. Process consent involved continuous monitoring of the validity of the informed consent provided at the beginning of the study, for example, verbal confirmation of consent at each time point with the formality of the process consent proportionate to the risks involved in participation.

Two studies reported the use of deferred consent in circumstances of fluctuating capacity with participants anticipated to regain capacity with reversibility of the underlying cause, for example, an infection [100, 104]. Patients who were unlikely to recover or die were typically excluded. Patients entered the study without their prior consent, with consent deferred until they regained capacity and/or a consultee was approached. These types of studies typically involved low-risk observational procedures conducted with, for example, older patients with delirium [100] or in the ICU [104]. Using deferred consent enabled the inclusion of participants at the acute point of their illness spectrum. Deferred consent appeared acceptable in the ICU study with an $80.1 \%(n=214 / 267)$ consent rate [104], and sensitivity 
analysis of the delirium study indicated that excluding ALC would have compromised the detection of statistically significant findings [100].

The main process of enabling adults lacking capacity to participate in research was seeking advice from a consultee about study participation. Most studies $(n=11)$ explored the involvement of consultees across research studies in geriatric/stroke care (all settings) $(n=7)$, ICU $(n=2)$, or mental health services $(n=1)$ (Table 3). Studies conferred that the role of a consultee was not to provide substituted judgement, but rather to give advice aligned with the patient's wishes and well-being. This required researchers to consult patients as much as possible about participation and encourage them to identify a consultee to advise on their behalf if they lost capacity [66]. Key to this process was discussion and shared decision-making between the consultee and the patient when they had capacity [67], and including potential consultees in early discussions with the person, while capacitous about future participation in research [47]. An observational study in ICUs involving patients $(n=$ 214) considered likely to regain capacity, and family members $(n=445)$ as surrogate decision-makers, reported that the only factor significantly associated with patients' confidence in their consultee's consent decision was having a previous conversation about research participation [105]. Consultees also reported reduced stress from their role when they could align their advice with understanding of the person's priorities [67]. However, a study involving nursing home residents reported no associations with patients' characteristics and consultee decision to advise enrolment $(54 \%, n=90)$ (or not) [47].

While consultees were generally family members (including close friends), consent processes also allowed professional consultees or legal representatives to advise on behalf of an individual lacking capacity. However, guidance and regulation around the participation of incapacitated adults in research and involvement of consultees varied by jurisdiction. An international study on stroke ( $n=1005$, across six European countries) [87] reported that one in three patients lacked capacity to consent. The study found considerable variation in the jurisdictions' respective enrolment requirements, detailing four different processes for adults lacking capacity as to who to approach as the proxy decision-maker (e.g. a legal guardian, next of kin, or independent physician consulting with the next of kin on the patient's presumed will). In the USA, institutional review boards (IRBs) were reported to differ in their rates of allowing research involving incapacitated adults regardless of the risks and benefits, and in who could act as a consultee within the studies [117]. For instance, 15\% of the IRBs disallowed participation in research without direct benefit regardless of risks, while $22 \%$ of IRBs accepted only an authorised proxy, spouse, or parent as surrogates, excluding adult children and other family members. Studies conducted with patients with dementia [68, 69] showed that requiring a legally appointed consultee led to declining participation from family members due to the bureaucracy and the time involved in the legal appointment. Such legal requirements could halt accumulation of evidence required for enhancing care of patients with impaired capacity.

Cluster consent was advocated for trials involving adults with impaired capacity in palliative care [48] and emergency medicine [106]. Cluster consent was undertaken by a 'cluster guardian' giving written agreement for treatment allocation of the defined cluster at the level of a unit, e.g. a hospital ward. This enabled the allocation of treatment at the cluster level. However, individual consent from the person, or consultee advice, was required for access to personal medical data for research purposes. Recommendations in emergency medicine research included enhancing recruitment using community consent as a similar process with 'group consent' for the study and individual 'opt-out' of the study at a later stage, for example, once capacity was regained using a similar process to deferred consent [106].

\section{Applying consent processes across the capacity spectrum in studies on serious illness}

Thirty-two publications reported 30 original studies involving adults with serious illness and impaired mental capacity $(n=9046)$ (see Table 4$)$. These studies were conducted across clinical settings (e.g. hospices, hospitals) and populations (e.g. elderly, dementia, delirium). Fourteen (46.6\%) studies were RCTs (including feasibility), and the remaining observational including prospective and cross-sectional designs. The studies enabled analysis on the use of different consent processes and the outcome of the recruitment rate for adults across the capacity spectrum in clinical studies.

Most studies were conducted in palliative care $(n=8)$ or dementia/geriatric care $(n=10)$. The studies used a breadth of consent processes tailored to the respective population. Methods included personal and/or professional consultee advice, advance and process consent, enhanced informed consent, and deferred consent. Processes showed variation in recruitment of eligible participants (range 23.9\% [72] to 78.8\% [42]). Use of a personal consultee alone (e.g. a family member) was uncommon and showed the greatest variability in the recruitment rate. Four studies used personal consultee advice only for adults lacking capacity $[49,50,83,108]$. The recruitment rates varied by study population and study design. In a cross-sectional survey exploring patients' palliative care needs in hospital, the overall recruitment rate was 48.1\% (654/1359) [49]. While most eligible patients 


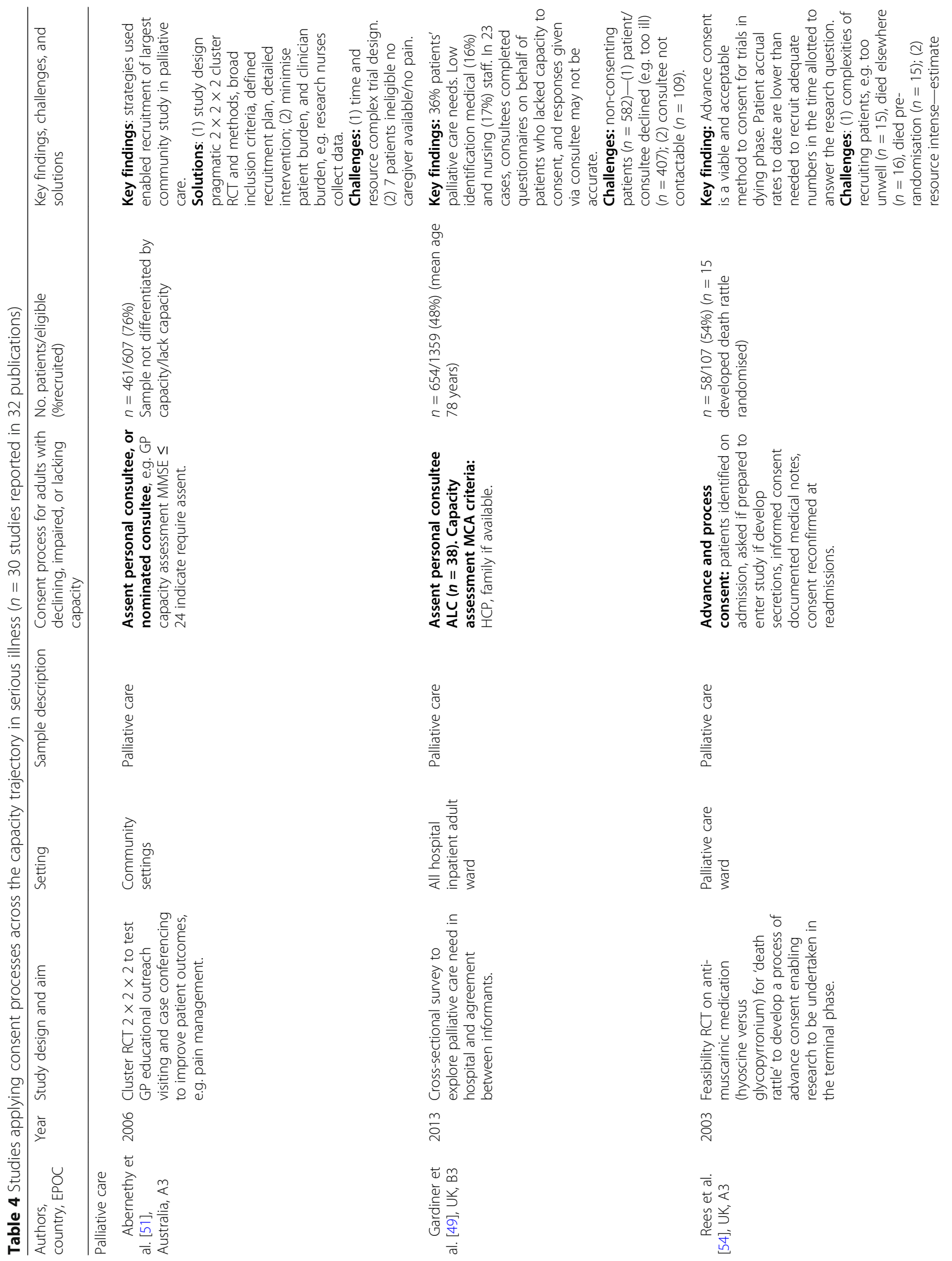




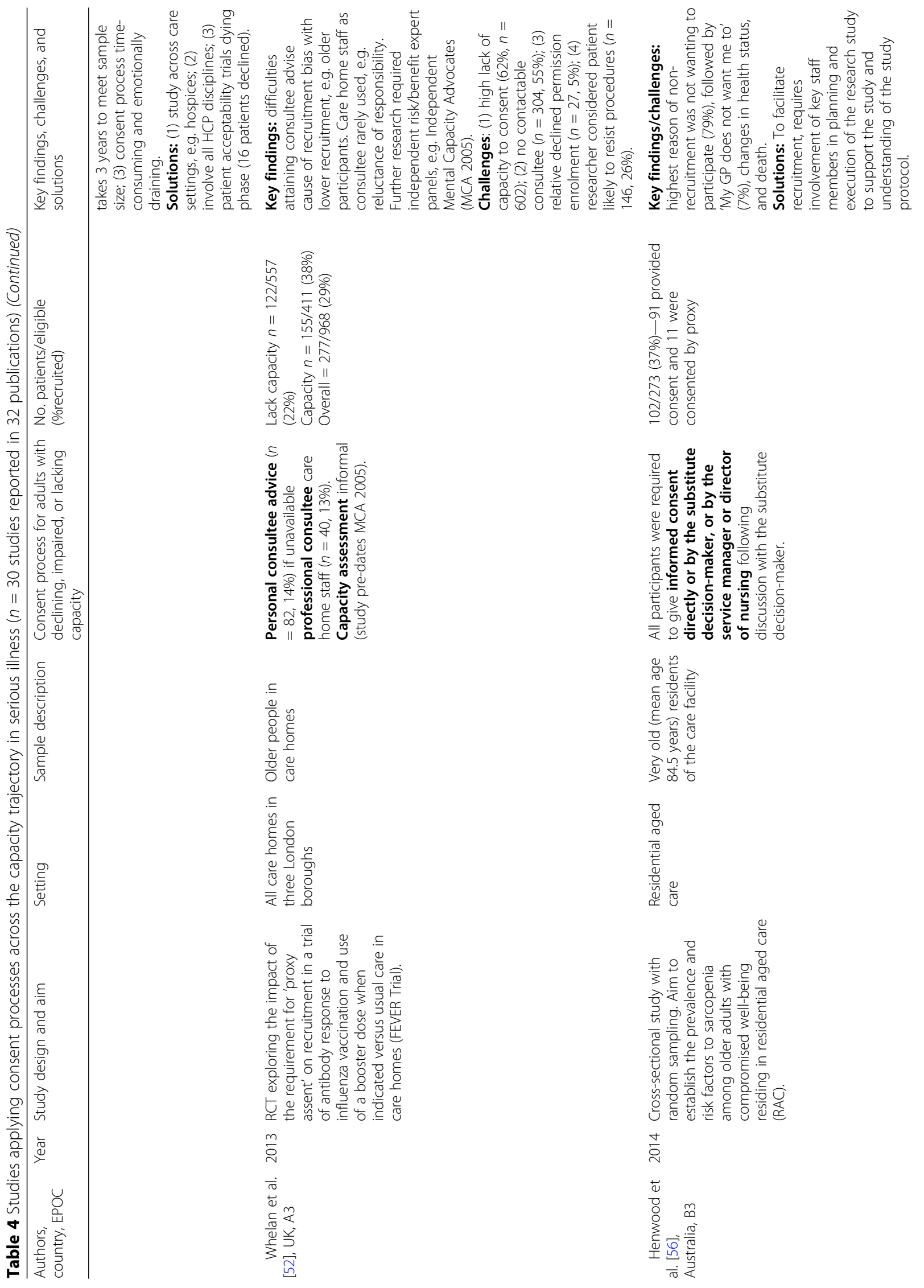




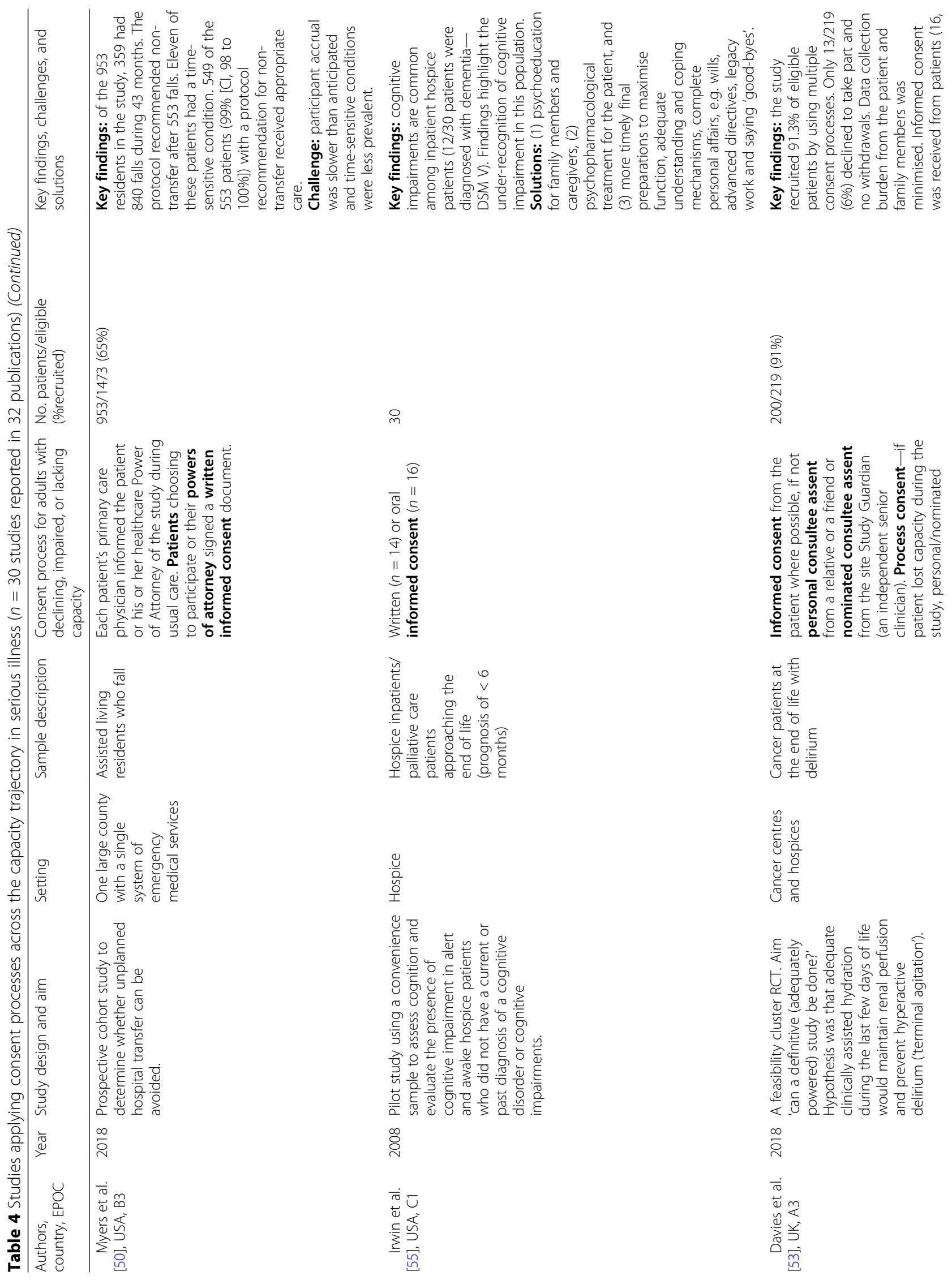




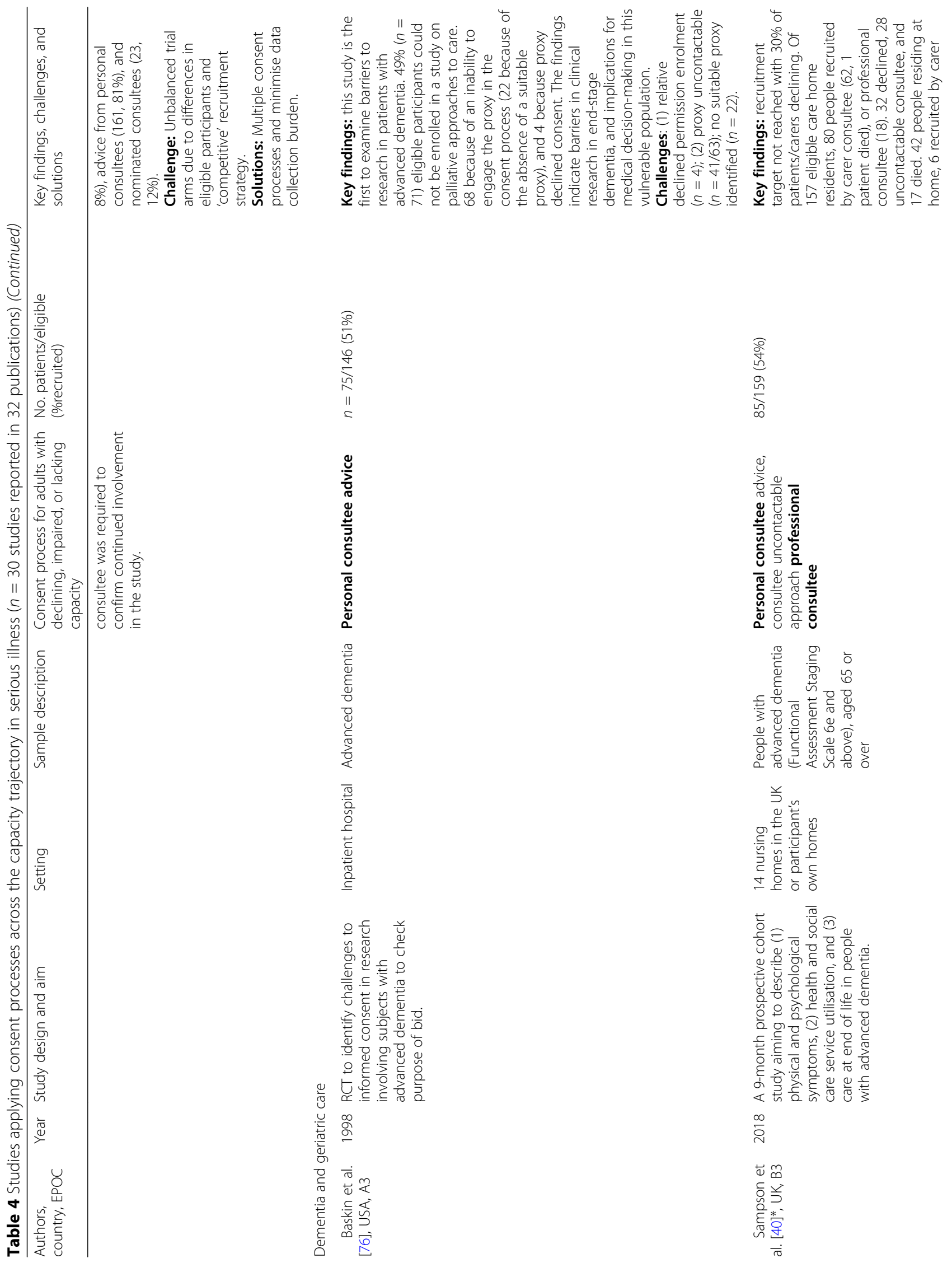




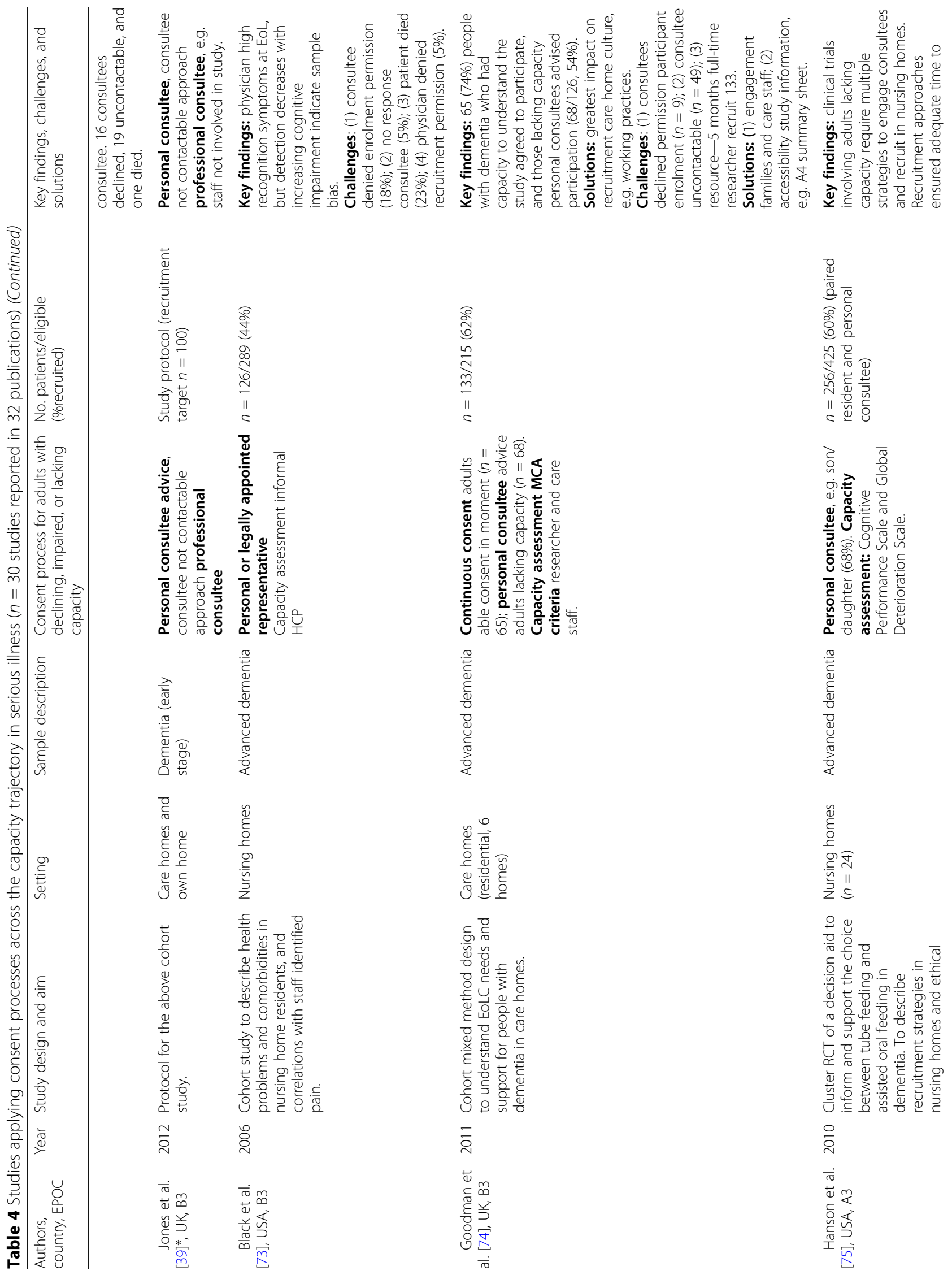




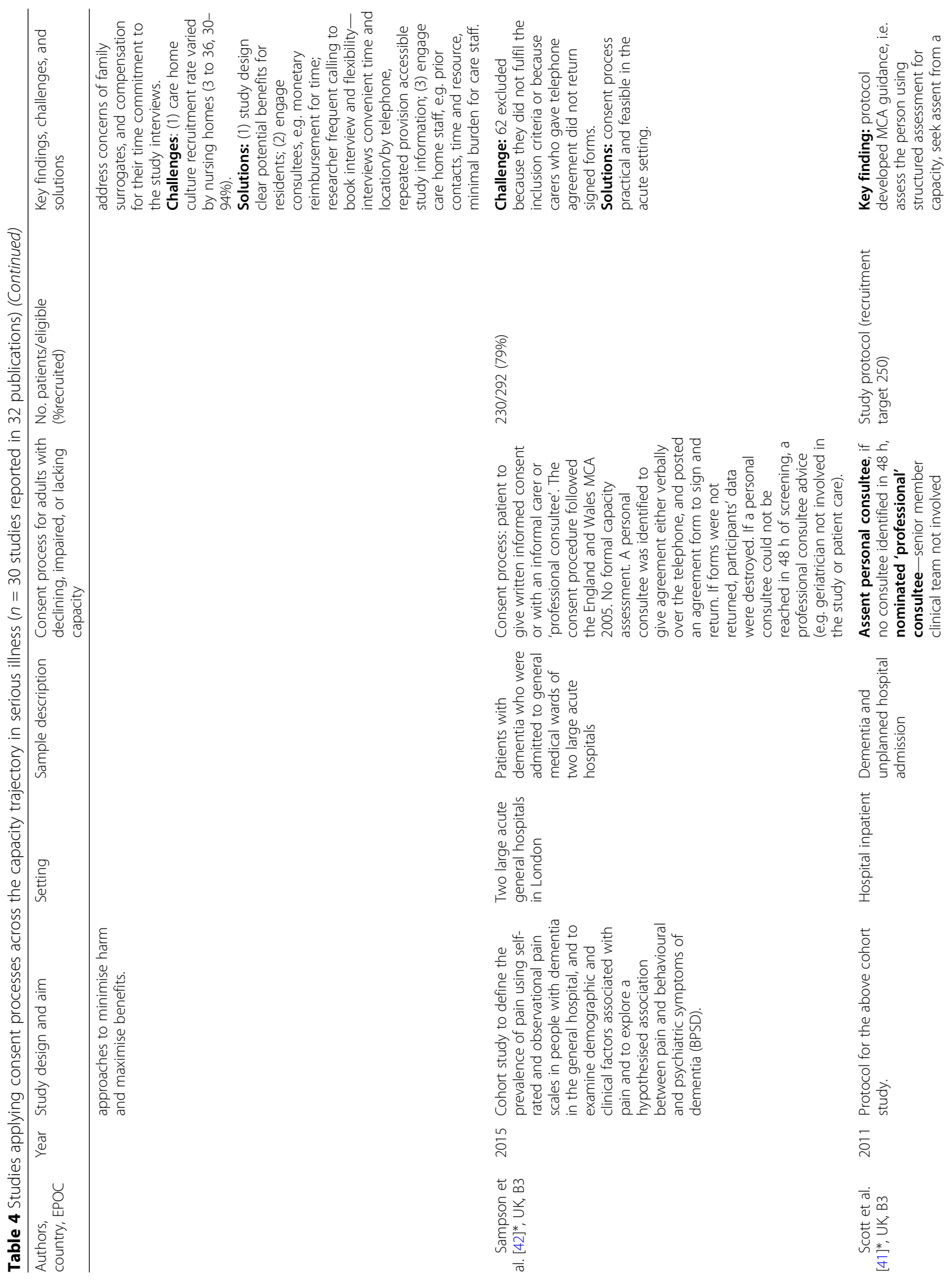




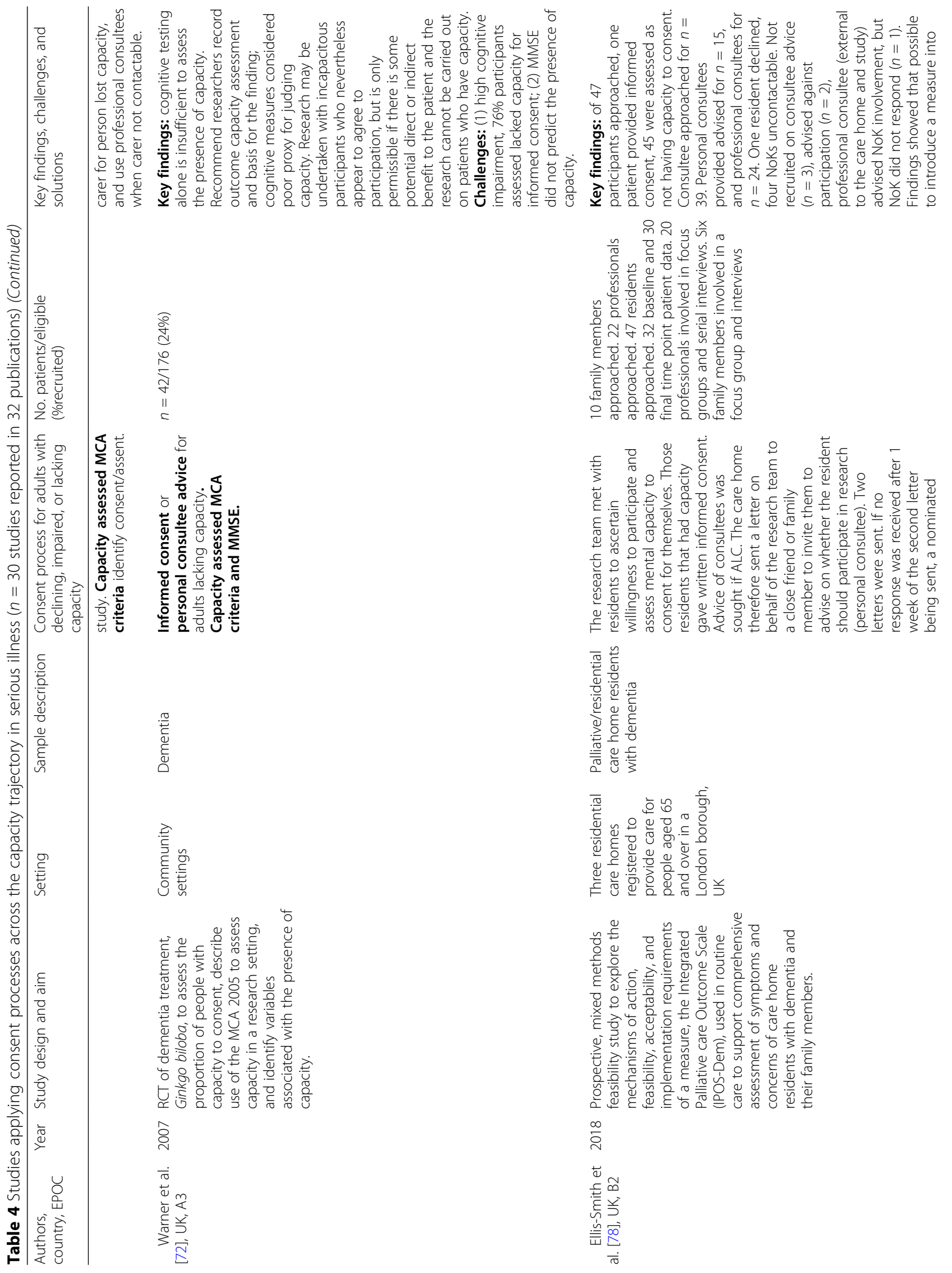




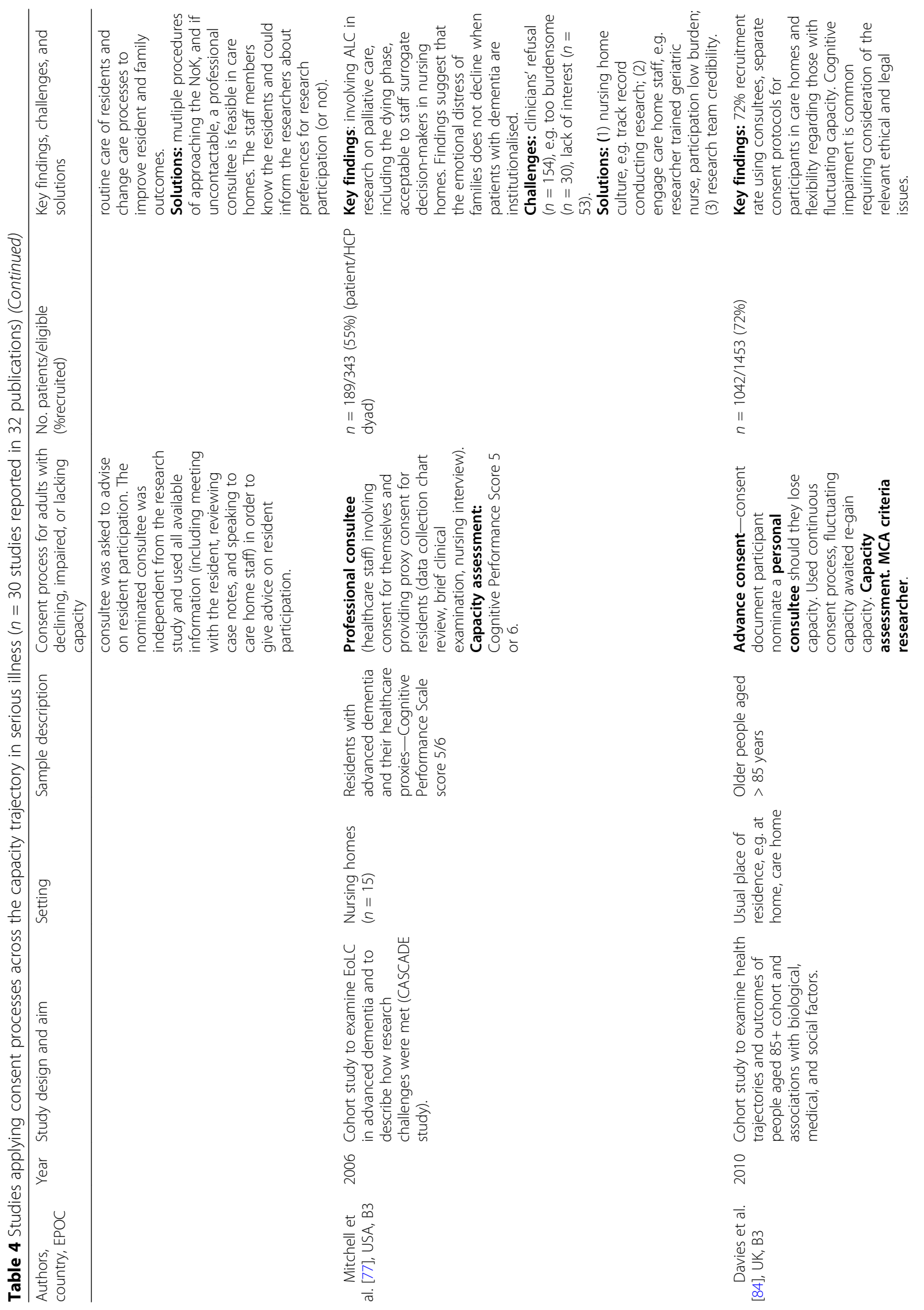




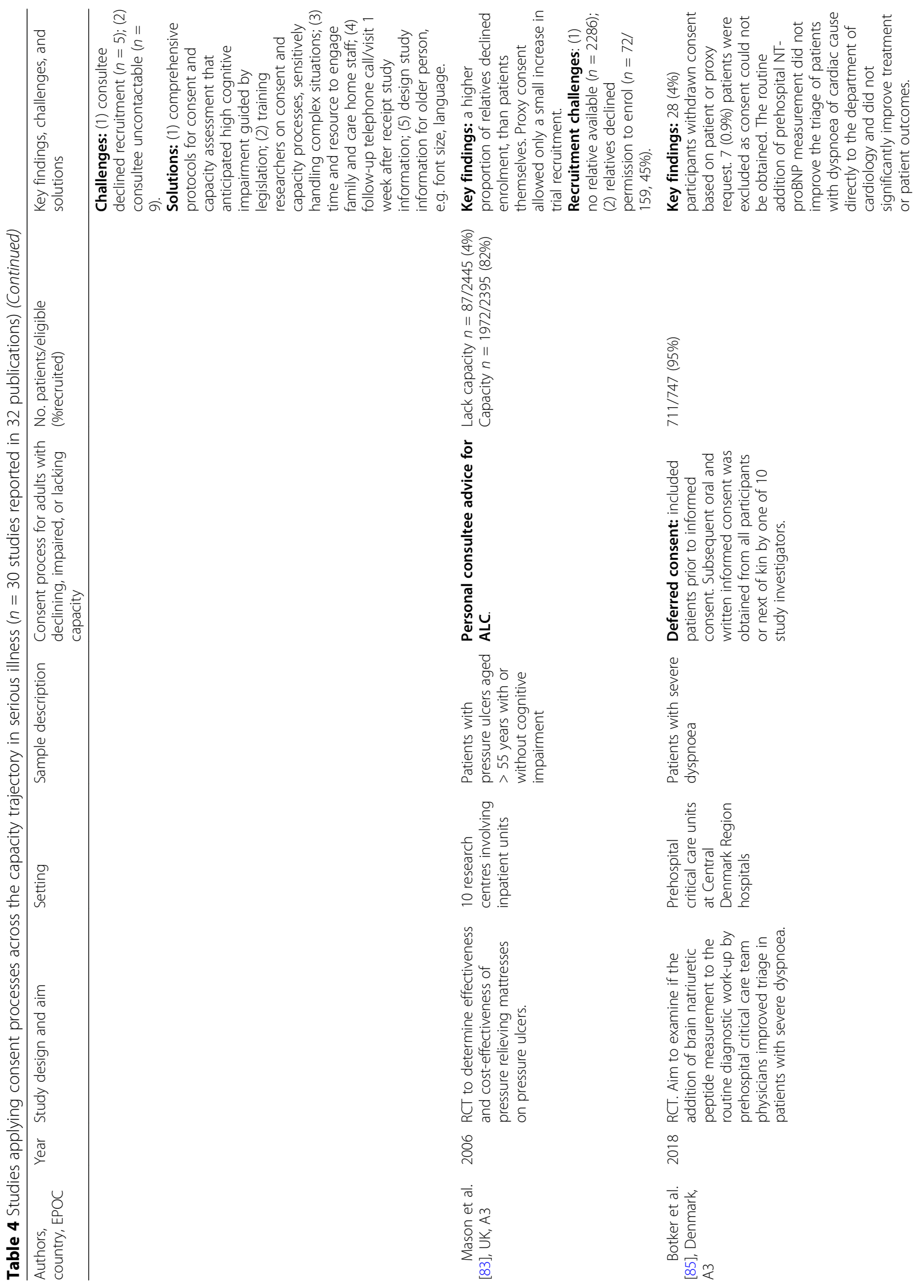




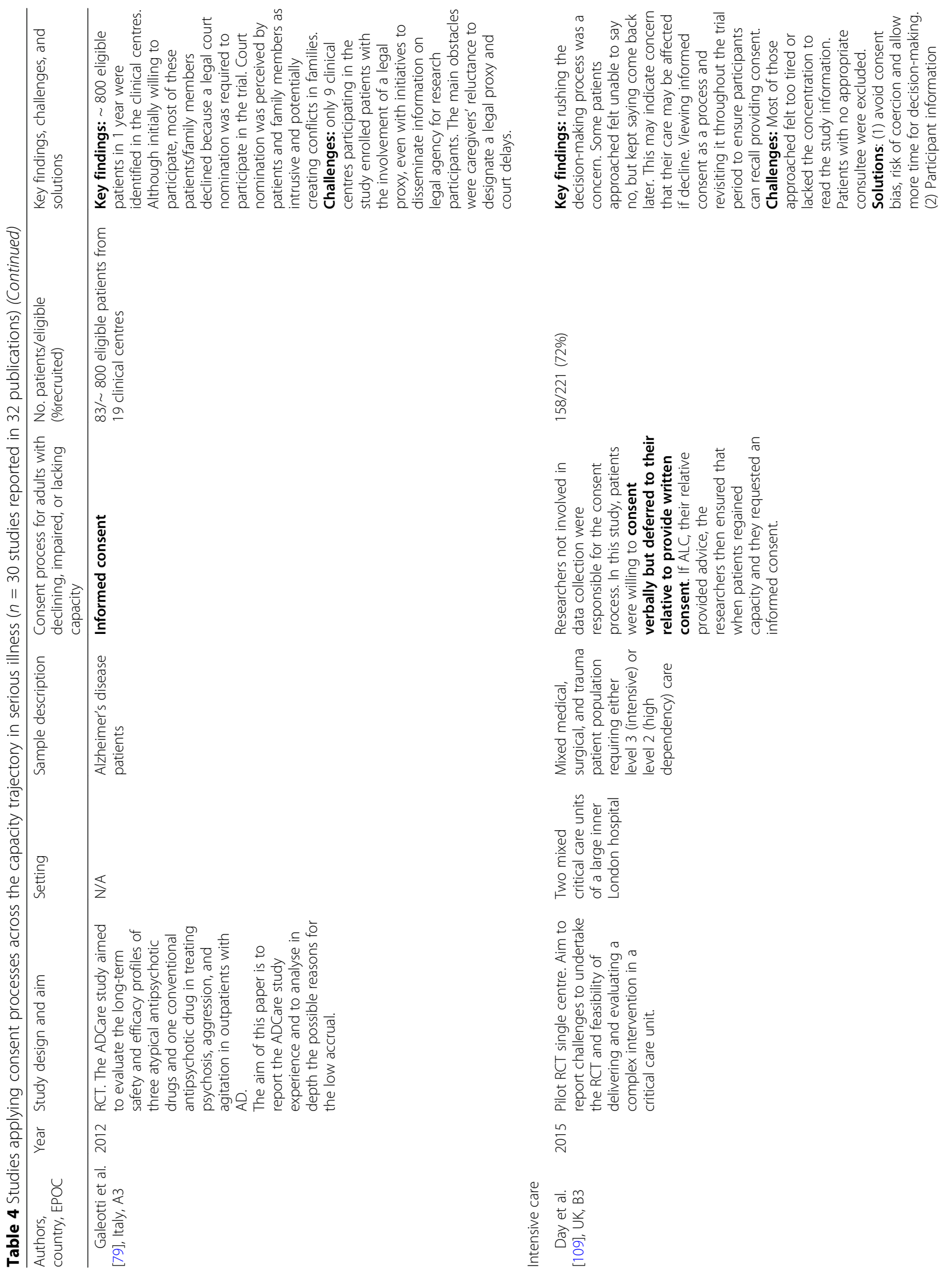




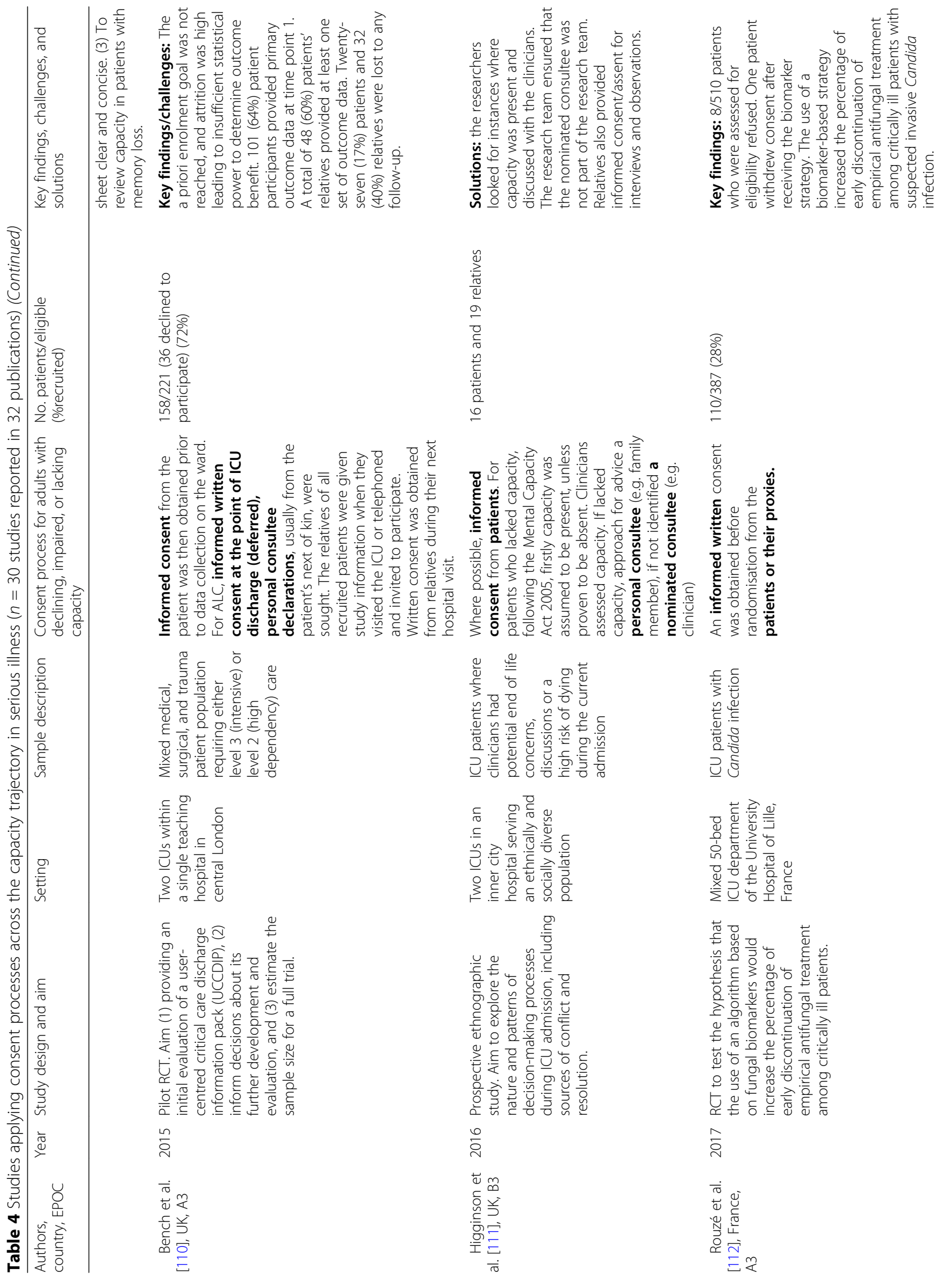




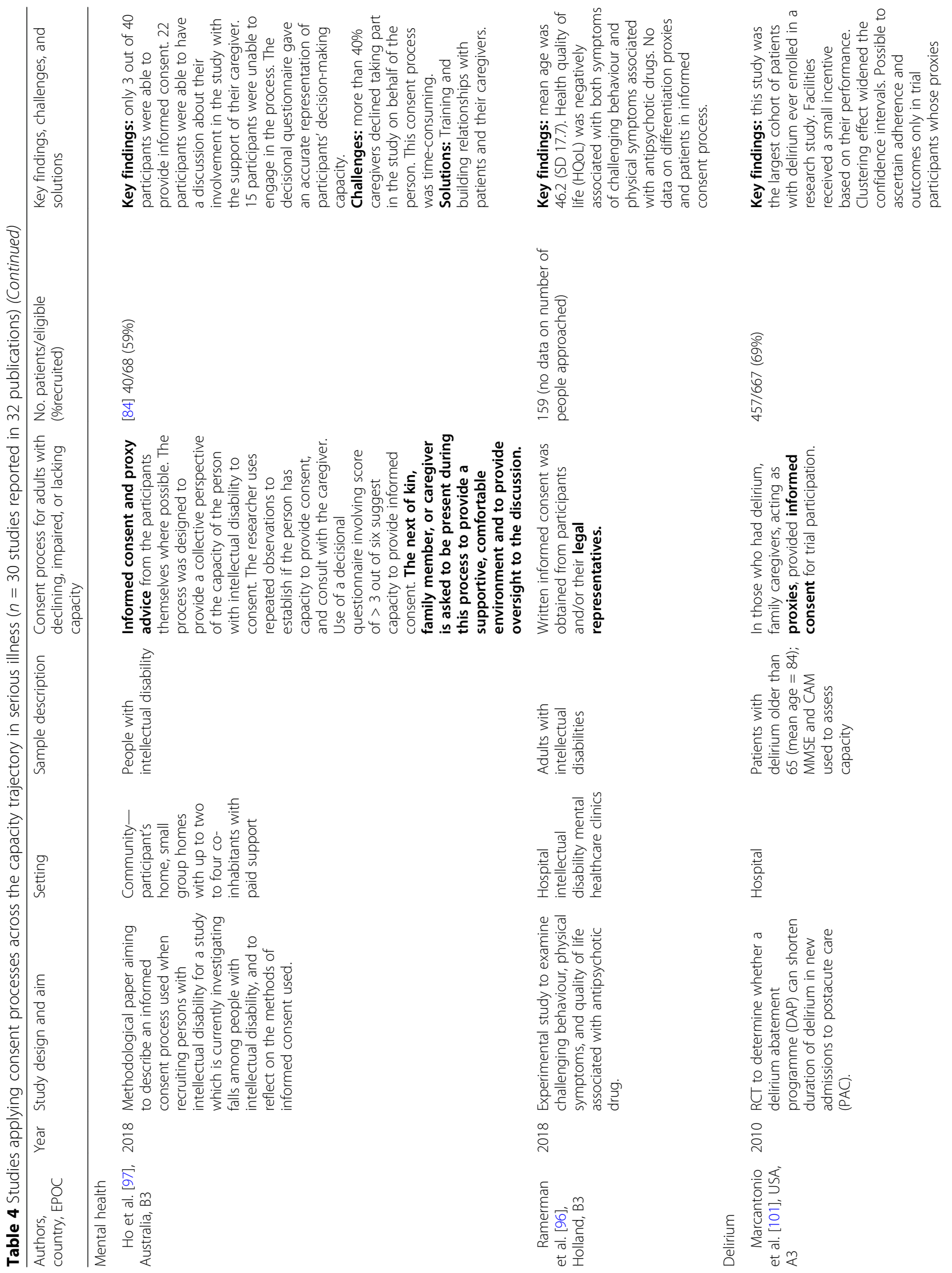




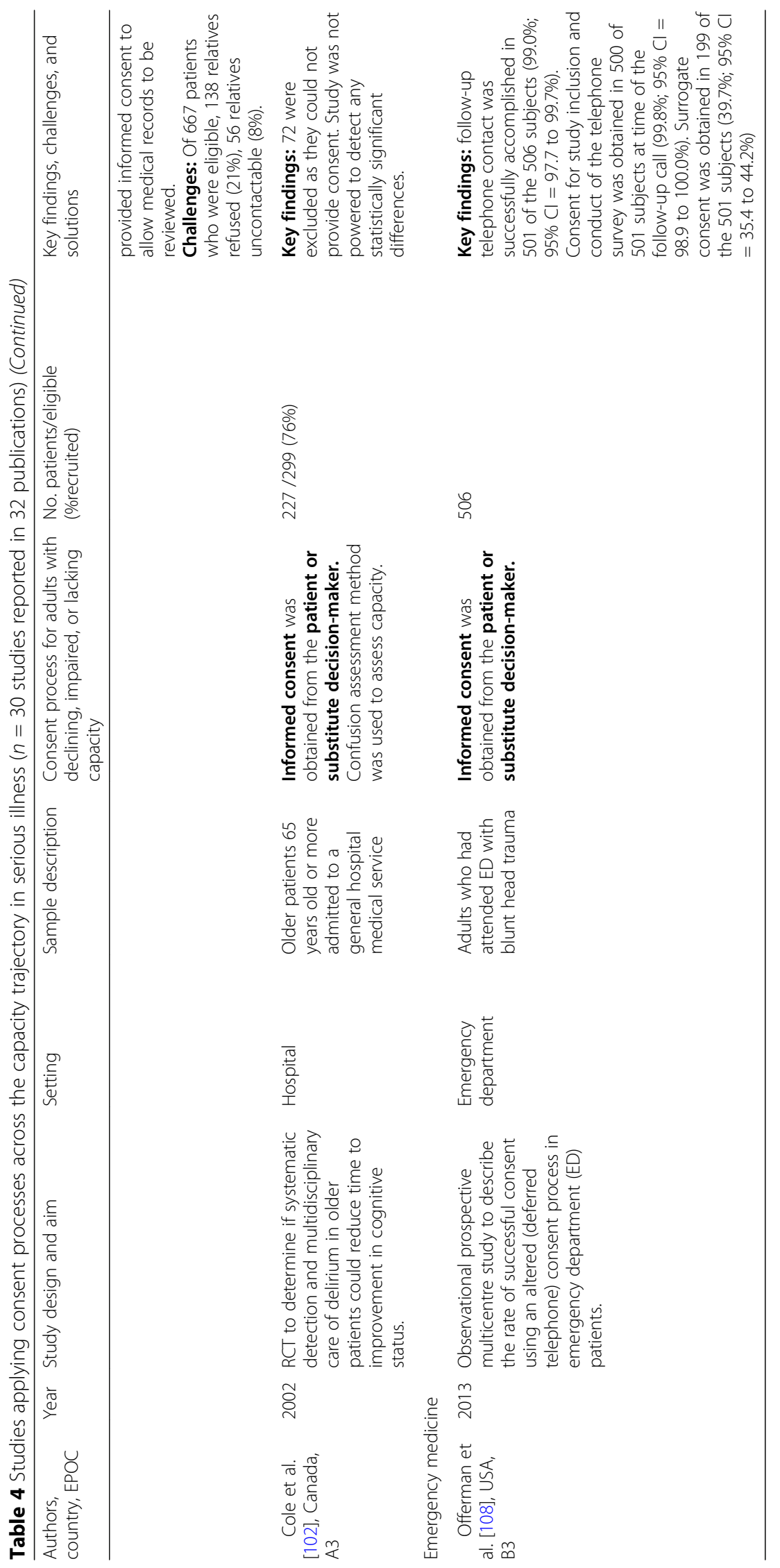


lacking capacity had an identifiable personal consultee able to advise, $8.1 \%$ of eligible patients $(n=109$ /1359) were not approached as no personal consultee was available. In contrast, in an RCT [83] on reducing pressure ulcers, only $3.6 \%(n=87 / 2445)$ of eligible patients who lacked capacity were recruited compared to $82.3 \%$ ( $n=$ 1972/2395) of patients able to giveinformed consent. The main reason for non-recruitment was no available personal consultee $(93.5 \%, 2286 / 2445)$, and when available, $45.3 \%$ declined to give advice $(n=72 / 159)$.

Three studies used both a personal and a professional consultee to provide advice with an initial approach to a personal consultee, and if unavailable an approach to a professional consultee [51-53]. Abernethy and colleagues [51] achieved a high recruitment rate $(n=461,75.9 \%)$ using this joint approach in a low-risk non-invasive cluster RCT on palliative care in community settings. A MMSE score of $\leq 24$ indicated the need for consultee advice. Only seven patients were ineligible due to consultee unavailability. The recruitment process used a resource-intensive defined recruitment plan that sought to facilitate recruitment by minimising patient and clinician burden. Similarly, a feasibility cluster RCT of clinically assisted hydration in cancer patients in the last days of life used multiple processes of informed consent for patients with capacity $(n=16,8 \%)$, and advice from a personal $(n=161$, $80.5 \%)$ or professional consultee $(n=23,11.5 \%)$ for adults lacking capacity, and process consent throughout the study [53]. The multiple consent processes enabled a 91.3\% recruitment rate $(n=200 / 219)$ of eligible patients from four cancer centres and eight hospices. Only 5.9\% $(n=13 / 219)$ of the eligible participants declined to participate, and none withdrew. Using clinical observations for data collection minimised burden for patients and family members. However, a competitive recruitment strategy caused variation in recruitment rate between clusters and imbalance in the trial arms $(n=73$ treatment arm and $n=$ 127 control arm). Conversely, an RCT of antibody response to influenza vaccination for older people in care homes incorporated multiple processes of consent [52]. But care home staff were hesitant to act as a professional consultee in the absence of a personal consultee. A total of 304 (54.5\%) eligible individuals were excluded for this reason, and recruitment of only $8.9 \%$ of eligible residents who required a professional consultee $(n=40 / 448)$. However, the study acceptable was mixed with $75.2 \%$ of personal consultee advising enrolment $(n=82 / 109)$, but only $37.7 \%$ of residents with capacity consented to recruitment $(n=155 / 411)$. Greater clarity on the responsibility of the professional consultee was required to enable care staff to act in this role, particularly in a trial involving invasive procedures.

Seeking advice from a consultee on enrolment was prominent in studies involving patients with dementia or delirium. Depending on the context of the study, recruitment rates ranged from $23.9 \%$ [72] to $78.8 \%$ [42]. Most studies in dementia took place in nursing homes/residential care homes [40, 42, 72-75], with only two RCTs conducted in hospital both on delirium [101, 102]. Common challenges to participation were relatives advising against patient enrolment $[40,73,101]$, unavailability of a personal consultee $[74,76,101]$, and care home staff acting as professional consultees declining to give advice for $44.9 \%$ of the eligible participants [77]. To overcome potential hesitancy from consultees required active engagement with consultees, keeping in touch and being flexible, highlighting potential benefits, and lowering burden for the consultee [75]. To minimise exclusion of eligible patients due to unavailability of personal consultees, studies used processes of a professional consultees and a clear recruitment strategy detailing the procedures $[42,78]$. A cohort study involving people with dementia from six care homes achieved $62.1 \%$ patient recruitment rate $(n=133 / 215)$ using a process of informed consent when possible $(n=65 / 89,73.8 \%)$ and personal consultees $(n=68 / 126,54 \%)$ [74]. The study reported the importance of engaging with patients and families by using a short summary sheet to enable understanding and participation. However, 49 eligible patients had no available personal consultee and were excluded [74].

Two studies $[54,84]$ in palliative care employed advance consent, followed by process consent (or personal consultee) processes. In a feasibility RCT of two medications for the treatment of death rattle, 54.2\% (58/107) of eligible patients with capacity provided advance consent. However, due to the complexities of the patient population and uncertain prognosis, only $25.9 \%$ (15/58) of patients were randomised [54]. The consent process was resource intensive to recruit the target sample size and emotionally draining for patients as it required conversations about an event which may not occur. Most patients accepted the advance consent process, with only 16 of 58 declining. A prospective cohort study examining health trajectories and outcomes in patients over 85 years old in usual place of residence achieved a $72.0 \%(1042 / 1453)$ recruitment rate [84]. Individuals who provided advance consent were also asked to nominate a personal consultee. Throughout the study, capacity of the recruited participants was assessed, and the nominated personal consultee was contacted if needed. Only five consultees declined, and nine were unavailable. The recruitment process was facilitated in multiple ways including using different consent and capacity assessment protocols for respective settings, tailoring the study information to the target population, flexibility towards those with fluctuating capacity, identifying personal consultees, training researchers, sensitively handling complex situations, and allocating time and resource to engage and keep in touch with family and care home staff. 
Four studies that took place in ICUs used informed consent and consultee advice, and deferred consent processes [109-112]. Pilot RCTs of complex interventions (non-invasive) used deferred consent processes [109, 110]. Patients agreed verbally to study enrolment, and their personal consultees gave written advice. Once patients recovered, they were approached for an informed consent [109]. The study achieved $71.5 \%$ recruitment rate. However, the possibility of coercion was raised with some patients indicating they felt unable to decline verbal enrolment, and consent bias with the exclusion of patients with no personal consultee. In another pilot RCT, the next of kin of $83.6 \%$ of patients who lacked capacity in the moment of data collection provided deferred advice at the point of discharge. Although this study had a $71.5 \%$ recruitment rate, $16.3 \%$ declined to participate. A further RCT involving both adults with capacity to provide informed consent and personal consultee advice for incapacitated patients showed only 8/510 eligible individuals declined participation [112]. Similarly, an RCT involving a blood test for patients with severe dyspnoea used deferred consent [85] and showed high acceptability with only $3.8 \%$ (28/747) withdrawing consent once regained capacity or on consultee advice. Only $0.9 \%$ (7/747) were excluded as unable to obtain deferred consent or consultee advice. An ethnographic observational study in ICU used informed consent and personal or professional consultee advice successfully to recruit 16 patients and 19 relatives with a clear consent protocol aligned to minimal risk of participation [111].

Enhanced consent processes were limited to studies involving adults with intellectual disabilities $[96,97]$ and in palliative care [55]. A study investigating falls in people with intellectual disability aimed to maximise an individual's ability to provide informed consent for themselves achieved a recruitment rate of $58.8 \%(n=40$ / 68). The study enhanced consent processes by involving family members and healthcare professionals in the process, using a questionnaire to assess capacity, and conducting multiple observations [97]. A personal consultee was sought for adults lacking capacity. However, over $40 \%$ of relatives advised non-participation in the study on behalf of the person as they considered the consent process too time-consuming and burdensome. A cross-sectional study on use of antipsychotic medication for people with intellectual disabilities recruited 159 eligible participants using informed consent and professional consultee comprising legal representative [96]. However, no denominator was stated. Finally, a pilot study of patients admitted to a hospice exploring levels of cognitive impairment sought to minimise the burden of informed by allowing written or oral informed consent [55].

\section{Public attitudes on enrolling adults lacking capacity in research}

Studies $(n=22)$ on attitudes towards enrolling adults lacking capacity in research consistently reported the acceptability of involving consultees to enable recruitment. However, the level of acceptability varied associated with the nature and purpose of the study (see Additional file 3: Table S8 reporting the studies' key findings, and suppl. 9 additional reporting [130-133]). Most studies (59.1\%) were conducted with public members (including researchers, healthy populations, IRBs) and used observational designs (e.g. survey) to explore attitudes about research with critically ill patients (e.g. conducted in intensive or emergency care settings), or focused on Alzheimer's disease, stroke, cancer, intellectual disabilities, overall incapacitated individuals, or proxy decision-making for study enrolment. Overall, stakeholders considered it acceptable to use substitute decision-making to enrol adults lacking capacity in research, especially for low-risk studies. Acceptability focused on prioritising a person's wishes and the potential benefits for the person, rather than the burden of acting as a proxy decision-maker.

\section{Transparent expert consultation}

The systematic review findings identified three critical areas debated in the TEC including:

1) Time and design of the consent process: 'How can we enhance the timeliness of the consent processes for adults with fluctuating or deteriorating capacity in research on palliative and EoLC?'

2) Enhancing consultee and supportive decisionmaking: 'How can we enhance proxy decisionmakers' role in the process of consent for adults who lack capacity?

3) Ethics, resources, and expertise: 'What are the key considerations in planning the consent process in studies involving adults lacking capacity and communicating this to a research ethics committee?'

The TEC stakeholder workshop involved 39 participants of the 83 invitees (47.0\%). The participants represented service users/lay voluntary sector representatives $(n=13,33.3 \%)$, researchers $(n=15,38.5 \%$ including ethicists), clinical academics $(n=9,23.1 \%)$, and clinicians $(n=25.1 \%)$. Voluntary sector representatives included lay members who were carers and/or patients living with a progressive condition recruited from charities for people with dementia (Age UK and Alzheimer's Society) and cancer/palliative care (Brainstrust, Marie Curie, Independent Cancer Patients' Voice, National Cancer Research Institute, and the then National Council for Palliative Care). The group generated 184 
recommendations on the three areas (area $1, n=60$ recommendations; area $2, n=72$ recommendations; and area $3, n=53$ recommendations). Following data analysis, 29 recommendations were presented in the online Delphi Survey round 1 (see Additional file 4: Table S10 Delphi participant characteristics). The recommendations pertained to timeliness and design of the consent process (area 1, $n=7$ recommendations), enhancing consultee and supportive decision-making (area 2, $n=10$ recommendations), and ethics resources and expertise (area $3, n=12$ recommendations) (see Additional file 4: Table S11 - Delphi round 1 recommendations and Fig. S12 - round 1 box and whisker plots). The Delphi survey round 1 involved the workshop participants $(n=39)$ and individuals unable to attend the workshop $(n=4)$. $51.8 \%$ participated $(n=43)$. Round 2 involved the round 1 respondents only $(83.7 \%$ response rate, $n=36)$. Findings from round 1 analysing 454 free-text comments informed revisions to 11 recommendations to reduce ambiguity. This mainly concerned the use of the term 'consultee' in relation to legislation. In round 2, consensus was apparent for 24 recommendations with strict/ broad agreement and five considered equivocal (see Additional file 4: Table S13 - Delphi round 2 recommendations and Fig. S14 - round 2 box and whisker plots). No recommendations were not indicated. The top recommendations by respective areas were as follows:

- Area 1. 'Information about a research study is comprehensible, short and written in accessible language' (R2, median 9, IQR 9-9) and 'Although the legal significance of advance consent will vary depending on the relevant legal framework, it is good practice for researchers to seek an advance consent while individuals have capacity to consent for themselves, for example, shortly after a diagnosis of a progressive illness' (R10, median 8, IQR 7-9)

- Area 2. 'The consent process is tailored to the individual's needs, capabilities and values with researchers observing for non-verbal and verbal cues that may indicate an individual may wish to withdraw' (R9, median 9, IQR9-9) and 'Advance care planning to include discussing and recording in a Statement of Wishes document an individual's nominated or personal consultee whose opinion on participating in a research study is sought if the patient loses capacity' (R21, median 8, IQR 7-8)

- Area 3. 'Researchers to demonstrate to Research Ethics Committees a clear process of consent for potential participants with compromised capacity that details how the researchers will proceed to tailor the consent process to maximise individuals' ability to consent for themselves and when and how they will seek an opinion from a consultee' (R29, median 9, IQR9-9) and 'Health and social care practitioners to recognise research as a core clinical activity in a similar way as teaching and training' (R23, median 9, IQR 9-9)

Three main areas were considered equivocal and were debated in the expert 'think-tank', and solutions proposed by consensus (see Additional file 4: Box S15 main areas and top solutions). Nineteen experts attended the think-tank, representing researchers/clinical academics $(n=12)$, clinicians $(n=2)$, lay voluntary sector representatives $(n=3)$, and PPI member $(n=1)$. The three equivocal areas comprised the following:

- Area 1. Involving and supporting consultees in the decision-making process with uncertainty on how best to support consultees to engage in the research process, with four recommendations indicating equivocal broad agreements (recommendations 6, 8, 15, 22, 27)

- Area 2. Practitioner training and education with agreement on the requirement to increase training and support to researchers and clinicians, but areas of uncertainty concerned for example how to provide and disseminate training and guidance, and how to fund (R5)

- Area 3. Legislative frameworks and the incorporation into research practice

A prominent equivocal area surrounded the Mental Capacity Act 2005 [29] legislation in England and Wales. The key solutions proposed concerned amendments in the supporting guidance for the Act to enable greater flexibility in the role of the 'professional' consultee in, for example, how they are identified. The Expert Panel concurred that the MCA process of review of an advance consent being upheld (or not) by a consultee if a participant lost capacity to consent should be applied to all clinical trials and not limited to trials of non-pharmacological interventions. This would include for example pharmaceutical trials where advance consent is upheld without requirement for consultee review if a person loses capacity in European legislation [134].

\section{Discussion}

This synthesis of evidence from systematic review and TEC identified challenges and solutions to including individuals across the capacity spectrum in research on EoLC. Our findings produce the MORECare_Capacity statement detailing 20 best practice solutions and implementation requirements to maximise study participation across the capacity spectrum (Table 5 and Fig. 3). The statement provides much needed guidance to maximise opportunities for adults across the capacity spectrum to participate in research. It is relevant for researchers, members of research ethical committees, individuals overseeing research governance, clinicians, the public, service users, and voluntary sector representatives. The statement details 
solutions for study designs at the participant level to maximise individual autonomy, enhance the contribution of proxy decision-makers, and ensure time and resources to enable participation and recruitment strategies that anticipate and plan for varying and changeable levels of capacity. At the structural level, key activities are engaging research ethical committees in how to include adults lacking capacity in research by auditing ethical approvals to review decision-making and inform research practice, and promoting the importance of research in health and social care to improve clinical care and raise awareness on recruiting adults from across the capacity spectrum to

Table 5 MORECare_Capacity statement solutions on recruiting adults with impaired mental capacity at the end of life in research

\begin{tabular}{l} 
Solutions \\
\hline 1. Researchers should design all aspects of the study in the context of potential risk, burden, and benefit of \\
study participation. \\
$\begin{array}{l}\text { 2. Institutional review boards and research ethical committees should have transparent decision-making pro- } \\
\text { cesses to ensure consistency on ethical approvals for studies from various health specialties involving adults } \\
\text { across the mental capacity trajectory. }\end{array}$
\end{tabular}

3. The individuals (e.g. relative, friend, formal carer, nursing home staff, healthcare staff) who can act as a consultee to advise on whether the individual would have wanted to participate in the research study had they had capacity should depend on the nature of the study, rather than legal restrictions.

4. Researchers should be able to demonstrate a clear process of consent for potential participants with compromised capacity that details how the researchers will tailor the consent process to maximise an individual's ability to consent for themselves and when and how they will seek an opinion from a consultee.

Maximising individual autonomy

5. Clinicians should engage in research participation conversations with patients at the early stages of illness, discussing varying levels of risk, burden, and benefit, and document the person's preferences and wishes in for example an advance directive.

6. Individuals who are likely to lose capacity should be asked to designate a consultee whose opinion on their participation in a research study will be sought if the individual loses capacity.

7. For individuals who have capacity to consent in the moment, but overtime may not remember the discussion, process consent should be adopted whereby researchers re-confirm the individual's wish to participate at each data collection time point.

8. Researchers should check for non-verbal (e.g. agitation) and verbal cues (e.g. I'm unsure why you are asking $\left.\mathrm{me}^{\prime}\right)$ that may indicate a wish to withdraw during the study.

Involving consultees

9. Personal consultees should be present in research participation conversations with patients at the early stages of illness.

10. Where possible, to improve social support, personal consultees/family members should be engaged in the (enhanced) informed consent process for adults with impaired capacity.

11. The nature and extent of the responsibility of a consultee acting on behalf of a patient should be clarified.

12. Establish a national body to provide support and information to family members/informal carers acting as personal consultees.

Tailoring recruitment process to need

Time

Enhancing the research culture and infrastructure
13. Researchers should incorporate in the study design research participation information and (where applicable) data collection tools in multiple formats (e.g. verbal, written, electronic).

14. Studies where potential participants are expected to be adults across the mental capacity trajectory should incorporate multiple consent processes (e.g. personal and professional consultees, informed and process consent).

15. When possible, potential participants should be allowed time for further discussions regarding their research participation decision.

16. The study design should allow time proportionate to the risk, burden, and benefit of participation for consent or consultee decision (before or after research participation if consent is deferred).

17. Researchers should build in the time required to engage with and train clinical staff who will be involved, and those who might act as professional consultees in the study.

18. Health and social care practitioners should recognise research as a core clinical activity in a similar way as teaching and training.

19. Clinicians should be supported and provided with training to ensure they are confident in their skills to discuss research studies with patients (and/or family members) during routine clinical contact.

20. Health and social care practitioners should support adults across the mental capacity trajectory at all stages of the research study by considering the person's best interests and individual wishes and preferences to uphold individual autonomy and minimise the risk of harm, and enable family members to act as a personal consultee by ensuring sufficient information and understanding about the role. 


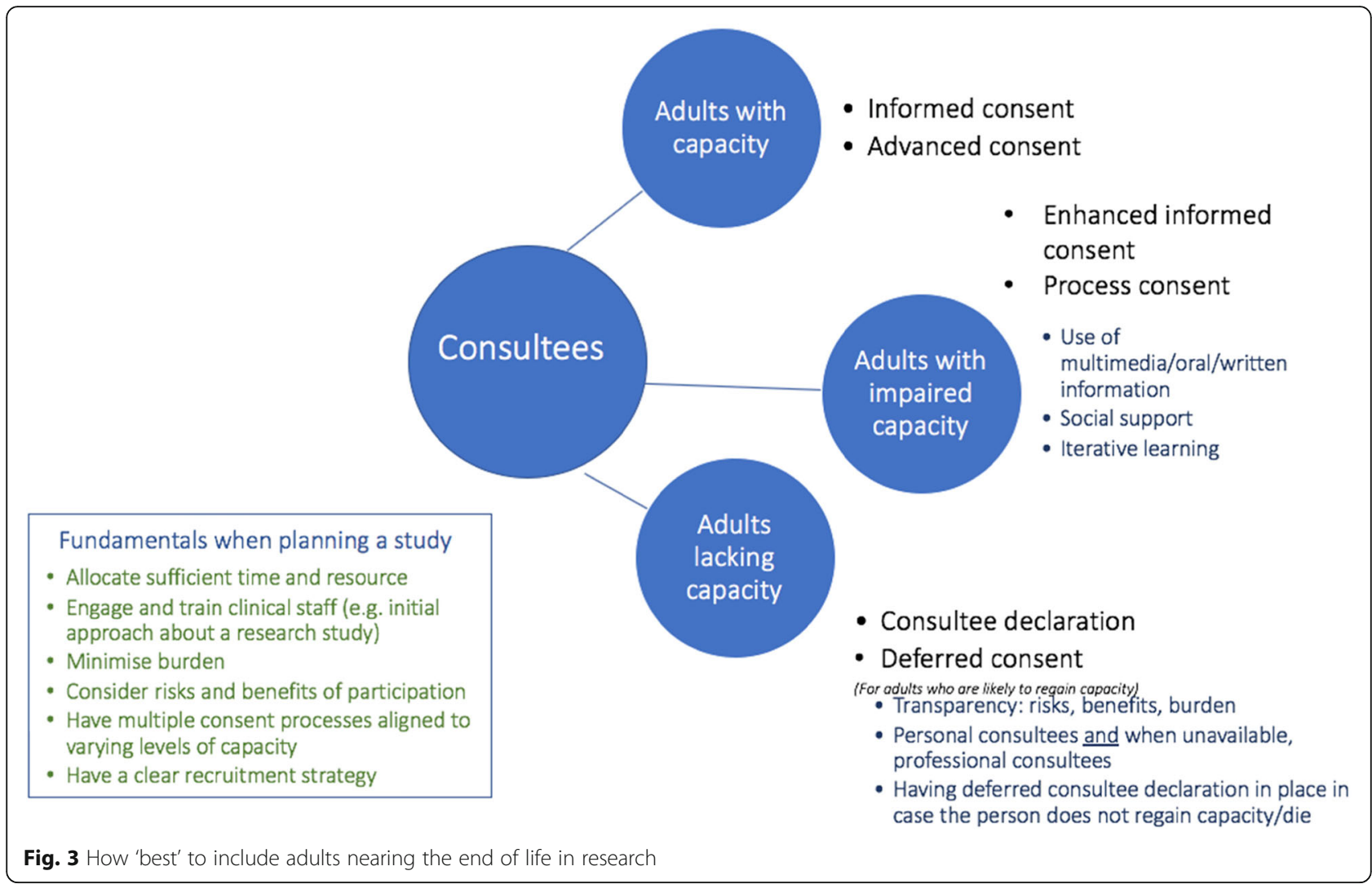

enhance the applicability of research findings for service users. The statement is intended as a framework for 'best' research practice. A statement cannot always anticipate change in an individual's circumstances or wishes. Ongoing review is always required by the consultee and researchers of an individual's best interests.

Our findings demonstrate that despite ethical challenges and legislative requirements, recruiting adults with impaired capacity in research is possible with careful consideration of the context, study design, and the resources required to enable participation. The main solutions for maximising research with incapacitated adults centred around early involvement of the person while they had capacity to indicate their preferences for research participation, and nomination and engagement with consultee(s) in these discussions. It is important to recognise that acting as either a personal (e.g. family member) or nominated (e.g. clinician) consultee can be burdensome, with burden increasing in studies which carry risks or little benefit the patient directly. A priority for consultees is to have sufficient time and information about their role and to have an awareness of the person's wishes through earlier discussions and documenting a person's wishes about research participation in, for example, an advance directive [21] or study specific in an advance consent. Figure 3 summarises the different processes across the capacity spectrum and sets out key factors to anticipate and plan for in a research study. Incorporation of our solutions in research studies on EoLC could increase the potential to recruit a representative sample of those intending to benefit from palliative care services and treatments. Involving patient groups with impaired capacity would increase generalisability of research findings by not limiting the evidence base to those with capacity and less advanced disease, and sustainability of the tested interventions in clinical care. Our solutions are informed by methodological and original research studies, and expert consensus. Importantly, surveys of the public and stakeholders consistently endorse the involvement of adults with impaired capacity in research and specifically in palliative care [135].

Ethically, research studies must endeavour to ask questions important for care and treatment, be designed to enable participation and minimise potential for harm, and demonstrate to research ethical committees a clear rationale for the study design and processes of consent for adults across the capacity spectrum (R29). In turn, ethical research committees must ensure transparency and consistency on ethical approvals for adults lacking capacity (R28). Working in this way seeks to uphold individual rights of, for example, autonomy to participate (or not), beneficence, and justice [136]. Beneficence in research on EoLC is important. Individuals with advanced disease are unlikely to benefit from the research 
findings seeking to improve care and treatment, but participation can enable a sense of contribution to society, of their 'voice' being valued and foster a sense of altruism [137]. Excluding individuals because of perceptions of vulnerability associated with advanced disease and impaired capacity may be considered unjust [3]. It denies equal opportunity for individuals living with progressive conditions to contribute to care and treatment in palliative care across the illness spectrum. To facilitate participation in research, our findings identify two key areas: 'enabling and empowering consultees' and 'a carefully constructed and resourced recruitment strategy'.

\section{Enabling and empowering consultees}

While techniques for enhancing autonomy are imperative to maximise individual decision-making, for adults with impaired capacity in EoLC studies, proxy decision-makers were crucial to enable recruitment. Consultees as proxy decision-makers sought to represent patients' wishes and best interests [105]. Their role was vital in enabling participation in research for adults across the capacity spectrum. While an array of consent processes can be implemented depending on the condition and level of capacity of the individual, successful participation of individuals relied on empowering and educating all those involved (patients, families, health and care staff, researchers, ethical committee members). Introducing patients to research studies while they were competent, and their family members, empowered patients and their families. Careful consideration is required to identity the right proxy decision-maker. Consultees sought to consider the benefits, risks, and burden to the person, and align their advice with understanding of the person's wishes. Earlier conversations enabled family members to align advice on participation with understanding on the person's wishes, particularly in comparatively high-risk studies [47, 67]. Similarly, West et al. in their systematic review on ethical challenges in dementia research reported differences in consultees advising enrolment with a higher proportion willing to provide consent for non-invasive studies compared to invasive studies, even with no potential direct benefit for the patient [21]. Our findings show that the presence of family members during enhanced consent processes enabled study participation by increasing opportunity and support for the person to discuss their priorities $[44,86]$. Although personal consultees were crucial, studies reported higher recruitment rates when they used a nominated/ professional consultee to advise if no personal consultee was available (compared to designs with a personal consultee alone). In contexts where being a professional consultee was unfamiliar, support and training for professional consultees were required to increase confidence and understanding of their role, for example, in a care home with little/no embedded research culture.

\section{A carefully constructed and resourced recruitment strategy}

Recruitment strategies in research on EoLC must align with the target population and anticipate and plan for varied and changeable levels of capacity associated with progressive disease. Even with strict eligibility criteria, potential participants within one study will likely present with varying and changeable levels of capacity. Our findings identify the necessity to have multiple consent processes in place aligned to the target population, context, and study aim to recruit individuals across the capacity spectrum [51, 53]. Studies on EoLC demonstrate the feasibility of using multiple processes in, for example, trials in the dying phase [54] and observational cohort studies [40,77]. The studies demonstrate the resource intensity and careful planning required to implement complex processes of advance consent when the person had capacity, and if/when capacity is lost, the involvement of proxy decision-makers (e.g. family or professional). Boland et al. echo these findings in their systematic review on recruitment strategies in trials involving people with serious illness [26]. Our findings show that where possible, researchers sought to maximise individual's autonomy, through consent processes such as enhanced, advanced, deferred, and process consent, and when lacked capacity incorporating a personal and/or professional consultee declaration that sought to align with understanding of the person's wishes and preferences. Enhanced consent was feasible and acceptable to augment understanding and reasoning by using methods of multiple formats and involving family members [61]. Similarly, Hostiuc et al. reported from their meta-regression that using multimedia to present study information significantly improved understanding, reasoning, and appreciation and enabled informed consent for adults with schizophrenia in clinical trials [22]. There is clearly opportunity to incorporate advances from other specialities, but with consideration if a successful strategy in one population is transferable to another. Different conditions impair different components of cognition, with variance in transience and progression (e.g. between schizophrenia and dementia), and prospect of recovery or not (e.g. between emergency medicine and palliative care). Further process evaluation of multimedia techniques is needed to determine the content and duration as the active ingredients of the techniques which leads to enhanced decisional capacity in studies for specific populations. Toolkits are useful resources to inform consent processes that encompass the heterogeneity of for example palliative care populations and the legislative framework for the respective jurisdiction and context [138] (See page 96 in for example [139]).

\section{Limitations}

Our incorporation of systematic review and expert consultation and consensus enabled consideration of a breadth of 
evidence and the application in study designs to enable participation across the capacity spectrum. The search strategy enabled consideration of a breadth of research methods employed in different clinical populations and contexts required to underpin the MORECare_Capacity statement. But the breadth of our strategy may have limited the identification of all relevant original research studies involving adults with serious illness and impaired capacity. While we present evidence from an array of original research studies including qualitative work, future work is required to systematically review recruitment processes for clinical trials in palliative care. The use of search terms to identify eligible studies was limited by poor reporting of recruitment processes and details regarding mental capacity of eligible participants [55]. To address this, we used supplementary processes of reference chaining and seeking recommendations from experts of key studies. In line with international reporting guidelines $[28,140]$, researchers should provide detailed information about recruitment processes and differentiate between participants who consented for themselves and those requiring a proxy decision-maker. This would improve assessment of the success of the recruitment methods used to inform research practice [55]. While recommended research methods can be generalisable, these need to be tailored to the respective setting, context, and culture. Our findings were limited to predominantly Western contexts. This reflects the concentration of palliative care research in, for example, the USA and Europe [141]. Finally, the study included lay representatives in the workshops and Delphi Survey, but PPI in the data synthesis and voluntary sector representation was limited to membership of the Project Advisory Group. Greater exploration is required in areas directly impacting on patients and families, for example, the use of advance directives to record a person's preferences for research participation (R21), and establishing a national body to provide support and information to family members acting as consultees (statement solution 12).

\section{Conclusions}

To meet the increasing need for palliative care requires greater provision of evidence-based services and treatments, which is informed by research that includes adults from across the capacity spectrum. Our findings show that conducting research involving adults with impaired capacity is feasible and acceptable, and it is ethically unjust to exclude them. Inclusion requires careful planning of processes of recruitment that are aligned to varying and changeable levels of capacity, and the nature and intent of the research study to minimise risk of harm. Studies must ask research questions important for patients and families in the provision of services, care, and treatment in serious healthrelated illness. This review and TEC present innovative research methods, solutions on enhancing use, and critical consideration on implementation in research studies in different populations, clinical settings, and research designs. The MORECare_Capacity statement provides solutions underpinned by carefully considered evidence on involving and supporting consultees, consent processes across the capacity spectrum, and decision-making with adaptations for the respective care setting. The solutions have applicability for vulnerable patient populations in palliative care and beyond such as mental health and emergency medicine. The application of the solutions stated requires consideration within respective jurisdiction's legislative framework. Future research is required on the applicability of the MORECare_C statement for non-Western cultures and low- to middle-income countries.

\section{Supplementary information}

Supplementary information accompanies this paper at https://doi.org/10 1186/s12916-020-01654-2.

Additional file 1: Table S1. PRISMA statement and checklist of items.

Additional file 2: Additional methods [Tables S2-S4]. Table S2: Systematic review electronic search terms for the respective database. Table S3: Study design categories using the Cochrane Effective Practice and Organisation of Care taxonomy. Table S4: Systematic review data extraction template.

Additional file 3: Additional results for the systematic review [Tables S5-S8; suppl. 9]. Table S5: Qualsyst quality assessment of the included quantitative studies. Table S6: Qualsyst quality assessment of the included qualitative studies. Table S7: Reported study designs categorised by the Cochrane Effective Practice and Organisation of Care taxonomy. Table S8: Public attitudes and ethical issues in recruiting adults across the capacity spectrum. Suppl. 9: Additional reporting on public attitudes and ethical issues.

Additional file 4: Additional results for the Transparent Expert Consultation [S10-S15]. Table S10: Delphi Survey participants in round one and round two. Table S11: Delphi Survey round one

recommendations and level of consensus. Fig. S12: Delphi Survey box and whisker plots round one recommendations. Table S13: Delphi Survey round two recommendations and level of consensus. Fig. S14: Delphi Survey box and whisker plots round two recommendations. Box S15: Expert 'think-tank' - equivocal areas and the priority solutions.

\section{Abbreviations \\ AD: Alzheimer's disease; A\&E: Accident and emergency; AMl: Acute myocardial infarction; CTIMP: Clinical Trial of an Investigational Medicinal Product; DMC: Decision-making capacity; EIC: Enhanced informed consent; EoLC: End of life care; EPOC: The Effective Practice and Organisation of Care; GP: General practitioner; HCP: Healthcare professional; ICU: Intensive care unit; IPOS-Dem: Integrated Palliative care Outcome Scale-Dementia; IRB: Institutional review board; LMICs: Low- to middle-income countries; Mac- CAT-CR: MacArthur competence assessment tool for clinical research; MCA: Mental Capacity Act 2005; MRC: Medical Research Council; MMSE: Mini- Mental State Examination; NoK: Next of kin; Non-CTIMP: Clinical Trial of an Investigational Non-medicinal Product; PRISMA: Preferred Reporting Items for Systematic Reviews and Meta-Analyses; QolC: Quality of informed consent; RCT: Randomised controlled trial; SD: Standard deviation; TEC: Transparent expert consultation}

\section{Acknowledgements}

We would like to thank our Project Advisory Group members: Deborah Tanner, Claire Henry, Gunn Grande, Steve Dewar, Gareth Owen, Rachel Burman, Dimitrios Adamis, Michael Dunn, Scott Kim, and Simon Woods for the oversight of the study and contributions, and Rowena Vohora for her administrative support in the initial stages of the study. 


\section{Authors' contributions}

$\mathrm{CJE}, \mathrm{PL}, J \mathrm{~K}, \mathrm{BW}, \mathrm{WB}, \mathrm{MH}$, and IJH were responsible for the conception and design of the study. CJE, EY, and KS were responsible for conducting the searches, data extraction, and analysis. CJE and KS were responsible for conducting and analysing the TEC, and EY in the reporting. CJE, EY, PL, JK, $\mathrm{KS}, \mathrm{IT}, \mathrm{BW}, \mathrm{WB}, \mathrm{MH}$, and IJH wrote and revised the manuscript. All authors read and approved the final manuscript.

\section{Funding}

MORECare_Capacity was funded by Marie Curie (MCCC-RP-11-A12544). MORECare_Capacity aimed to determine how best to include individuals near to death in research on EoLC by identifying solutions and developing best practice guidance on processes of consent for people with impaired mental capacity. CJE is funded by HEE/NIHR Senior Clinical Lectureship (ICASCL-2015-01-001). The views expressed are those of the author(s) and not necessarily those of the NIHR, the Department of Health and Social Care. The funders had no role in the design of the study and collection, analysis, and interpretation of data and in writing the manuscript.

\section{Availability of data and materials}

All data analysed for this review are included in this published article and its additional files.

\section{Ethics approval and consent to participate}

The transparent expert consultation received ethical review and approval from King's College London Research Ethics Committee (ref no. BDM/10/1190). All participants who took part provided informed consent. The systematic review included previously published studies. We could not influence the design of the prior studies upon which this work is based and cannot comment on individual ethics approval or consent.

\section{Consent for publication}

Not applicable.

\section{Competing interests}

The authors declare that they have no competing interests.

\section{Author details}

${ }^{1}$ Cicely Saunders Institute of Palliative Care, Policy \& Rehabilitation, Florence Nightingale Faculty of Nursing, Midwifery \& Palliative Care, King's College London, Bessemer Road, London SE5 9PJ, UK. ${ }^{2}$ Sussex Community NHS Foundation Trust, Brighton General Hospital, Brighton, UK. ${ }^{3}$ Centre of Medical Law and Ethics, The Dickson Poon School of Law, King's College London, London, UK. ${ }^{4}$ Oxford University Hospitals NHS Foundation Trust and Harris Manchester College, University of Oxford, Oxford, UK. ${ }^{5}$ King's College Hospital, London, UK. ${ }^{6}$ Sychological Medicine, Institute of Psychiatry, Psychology \& Neuroscience, King's College London, London, UK.

Received: 12 December 2019 Accepted: 3 June 2020

Published online: 22 July 2020

\section{References}

1. Sleeman KE, de Brito M, Etkind S, Nkhoma K, Guo P, Higginson IJ, Gomes B, Harding R. The escalating global burden of serious health-related suffering: projections to 2060 by world regions, age groups, and health conditions. Lancet Glob Health. 2019;7(7):e883-92.

2. Knaul FM, Farmer PE, Krakauer EL, De Lima L, Bhadelia A, Jiang Kwete X, Arreola-Ornelas H, Gomez-Dantes O, Rodriguez NM, Alleyne GAO, et al. Alleviating the access abyss in palliative care and pain relief-an imperative of universal health coverage: the Lancet Commission report. Lancet. 2017; 391(10128):1391-454.

3. Koffman J, Morgan M, Edmonds P, Speck P, Higginson IJ. Vulnerability in palliative care research: findings from a qualitative study of black Caribbean and white British patients with advanced cancer. J Med Ethics. 2009;35(7):440-4.

4. Department of Health. End of Life Care Strategy - promoting high quality care for adults at the end of life. London: Crown; 2008.

5. Neuberger J, Guthrie C, Aaronovitch D. More care, less pathway: a review of the Liverpool Care Pathway. London: Department of Health and Social Care; 2013.

6. Koffman J, Yorganci E, Yi D, Gao W, Murtagh F, Pickles A, Barclay S, Johnson $\mathrm{H}$, Wilson R, Sampson L, et al. Managing uncertain recovery for patients nearing the end of life in hospital: a mixed-methods feasibility cluster randomised controlled trial of the AMBER care bundle. Trials. 2019;20(1):506.

7. Goodwin DM, Higginson IJ, Edwards AG, Finlay IG, Cook AM, Hood K, Douglas HR, Normand CE. An evaluation of systematic reviews of palliative care services. J Palliat Care. 2002;18(2):77-83.

8. Lorenz KA, Lynn J, Dy SM, Shugarman LR, Wilkinson A, Mularski RA, Morton SC, Hughes RG, Hilton LK, Maglione M, et al. Evidence for improving palliative care at the end of life: a systematic review. Ann Intern Med. 2008; 148(2):147-59

9. Zimmermann C, Riechelmann R, Krzyzanowska M, Rodin G, Tannock I. Effectiveness of specialized palliative care: a systematic review. JAMA. 2008; 299(14):1698-709.

10. Gysels M, Higginson IJ. Improving supportive and palliative care for adults with cancer: research evidence. London: NICE; 2004.

11. Finlay IG, Higginson IJ, Goodwin DM, Cook AM, Edwards AG, Hood K, Douglas HR, Normand CE. Palliative care in hospital, hospice, at home: results from a systematic review. AnnOncol. 2002;13(Suppl 4):257-64.

12. Chan RWJ. End-of-life care pathways for improving outcomes in caring for the dying. Chichester: Cochrane Database of Systematic Reviews: Reviews 2010 Issue 1 John Wiley \& Sons; 2010.

13. Higginson IJ, Finlay IG, Goodwin DM, Hood K, Edwards AG, Cook A, Douglas HR, Normand CE. Is there evidence that palliative care teams alter end-oflife experiences of patients and their caregivers? J Pain Symptom Manag. 2003;25(2):150-68.

14. Shepherd V, Griffith R, Sheehan M, Wood F, Hood K. Healthcare professionals' understanding of the legislation governing research involving adults lacking mental capacity in England and Wales: a national survey. J Med Ethics. 2018;44(9):632-7.

15. Casarett DJ, Karlawish JH. Are special ethical guidelines needed for palliative care research? J Pain Symptom Manag. 2000;20(2):130-9.

16. Dewing J. From ritual to relationship: a person centred approach to consent in qualitative research with older people who have dementia. Int I Soc Res Pract. 2002; (2):156-71.

17. Dewing J. Participatory research: a method for process consent with persons who have dementia. Dementia. 2007;6(1):11-25.

18. Nuffield Council on Bioethics. Dementia ethical issues. London: Nuffield Council on Bioethics; 2009.

19. Owen GS, David AS, Richardson G, Szmukler G, Hayward P, Hotopf M. Mental capacity, diagnosis and insight in psychiatric in-patients: a crosssectional study. Psychol Med. 2009;39(8):1389-98.

20. Lemaire F, Blanch L, Cohen SL, Sprung C. Informed consent for research purposes in intensive care patients in Europe--part II. An official statement of the European Society of Intensive Care Medicine. Working group on ethics. Intensive Care Med. 1997;23(4):435-9.

21. West E, Stuckelberger A, Pautex S, Staaks J, Gysels M. Operationalising ethical challenges in dementia research--a systematic review of current evidence. Age Ageing. 2017;46(4):678-87.

22. Hostiuc S, Rusu MC, Negoi I, Drima E. Testing decision-making competency of schizophrenia participants in clinical trials. A meta-analysis and metaregression. BMC Psychiatry. 2018;18:2.

23. Mukherjee A, Livinski AA, Millum J, Chamut S, Boroumand S, lafolla TJ, Adesanya MR, Dye BA. Informed consent in dental care and research for the older adult population. J Am Dental Assoc. 2017;148(4):211-20.

24. Sugarman J, McCrory DC, Hubal RC. Getting meaningful informed consent from older adults: a structured literature review of empirical research. J Am Geriatr Soc. 1998;46(4):517-24.

25. Wohleber AM, McKitrick DS, Davis SE. Designing research with hospice and palliative care populations. Am J Hosp Palliat Med. 2012;29(5):335-45.

26. Boland J, Currow DC, Wilcock A, Tieman J, Hussain JA, Pitsillides C, Abernethy AP, Johnson MJ. A systematic review of strategies used to increase recruitment of people with cancer or organ failure into clinical trials: implications for palliative care research. J Pain Symptom Manag. 2015;49(4):762-72 e765.

27. Bruera E, Hui D, Dalal S, Torres-Vigil I, Trumble J, Roosth J, Krauter S, Strickland C, Unger K, Palmer JL. Parenteral hydration in patients with advanced cancer: a multicenter, double-blind, placebo-controlled randomized trial. J Clin Oncol. 2013;31(1):111.

28. Higginson IJ, Evans CJ, Grande G, Preston Nancy J, Morgan M, McCrone P, Lewis P, Fayers P, Harding R, Hotopf M, et al. Evaluating complex interventions in End of Life Care: the MORECare Statement on good practice generated by a synthesis of transparent expert consultations and systematic reviews. BMC Med. 2013;11(1):111 https://doi.org/10.1186/1741-7015-11-111(111). 
29. Department of Health. Mental Capacity Act. London: Department of Health; 2005.

30. Association WM. World Medical Association Declaration of Helsinki. Ethical principles for medical research involving human subjects. Bull World Health Organ. 2001;79(4):373.

31. Fink A, Kosecoff J, Chassin M, Brook R. Consensus methods: characteristics and guidelines for use. Am J Public Health. 1984;74(9):979-83.

32. Sinha IP, Smyth RL, Williamson PR. Using the Delphi technique to determine which outcomes to measure in clinical trials: recommendations for the future based on a systematic review of existing studies. PLoS Med. 2011; 8(1):e1000393.

33. Pope C, Mays N, Popay J. Synthesizing qualitative and quantitative health evidence: a guide to methods. Maidenhead and New York: Open University Press, McGraw Hill Education; 2007.

34. Moher D, Shamseer L, Clarke M, Ghersi D, Liberati A, Petticrew M, Shekelle P, Stewart LA. Preferred reporting items for systematic review and metaanalysis protocols (PRISMA-P) 2015 statement. Syst Rev. 2015;4(1):1.

35. Kmet LM, Lee RC, Cook LS. Standard quality assessment criteria for evaluating primary research papers from a variety of fields: Alberta Heritage Foundation for Medical Research; 2004.

36. EPOC. Cochrane effective practice and organisation of care review group. Data collection checklist. In. Ottowa: Cochrane Effective Practice and Organisation of Care; 2002.

37. Endnote X8. http://endnote.com/.Accessed 2 Nov 2016.

38. Jones J, Hunter D. Consensus methods for medical and health services research. In: Mays N, Pope C, editors. Qualitative research in health care. 2nd ed. London: British Medical Journal Publishing Group; 1999.

39. Jones L, Harrington J, Scott S, Davis S, Lord K, Vickerstaff V, Round J, Candy B, Sampson EL. CoMPASs: IOn programme (Care Of Memory Problems in Advanced Stages of dementia: Improving Our Knowledge): protocol for a mixed methods study. BMJ Open. 2012;2(6).

40. Sampson EL, Candy B, Davis S, Gola AB, Harrington J, King M, Kupeli N, Leavey G, Moore K, Nazareth I. Living and dying with advanced dementia: a prospective cohort study of symptoms, service use and care at the end of life. Palliat Med. 2018;32(3):668-81.

41. Scott S, Jones L, Blanchard MR, Sampson EL. Study protocol: the behaviour and pain in dementia study (BePAID). BMC Geriatr. 2011;11:61.

42. Sampson EL, White N, Lord K, Leurent B, Vickerstaff V, Scott S, Jones L. Pain, agitation, and behavioural problems in people with dementia admitted to general hospital wards: a longitudinal cohort study. Pain. 2015;156(4):675-83.

43. Dobratz MC. Issues and dilemmas in conducting research with vulnerable home hospice participants. J Nurs Scholarsh. 2003;35(4):371-6.

44. Chouliara Z, Kearney N, Worth A, Stott D. Challenges in conducting research with hospitalized older people with cancer: drawing from the experience of an ongoing interview-based project. Eur J Cancer Care. 2004;13(5):409-15.

45. Gysels M, Evans CJ, Lewis P, Speck P, Benalia H, Preston NJ, Grande GE, Short V, Owen-Jones E, Todd CJ. MORECare research methods guidance development: recommendations for ethical issues in palliative and end-oflife care research. Palliat Med. 2013;27(10):908-17.

46. Dunning T, Duggan N, Savage S, Martin P. Diabetes and end of life: ethical and methodological issues in gathering evidence to guide care. Scand $J$ Caring Sci. 2013;27(1):203-11.

47. Warren JW, Sobal J, Tenney JH, Hoopes JM, Damron D, Levenson S, DeForge BR, Muncie HL Jr. Informed consent by proxy. N Engl J Med. 1986; 315(18):1124-8.

48. Fowell A, Johnstone R, Finlay IG, Russell D, Russell IT. Design of trials with dying patients: a feasibility study of cluster randomisation versus randomised consent. Palliat Med. 2006;20(8):799-804.

49. Gardiner C, Gott M, Ingleton C, Seymour J, Cobb M, Noble B, Bennett M, Ryan $T$. Extent of palliative care need in the acute hospital setting: a survey of two acute hospitals in the UK. Palliat Med. 2013;27(1):76-83.

50. Myers JB. Improving decisions about transport to the emergency department for assisted living residents who fall. Ann Intern Med. 2018;168(3):179-86.

51. Abernethy AP, Currow DC, Hunt R, Williams H, Roder-Allen G, Rowett D, Shelby-James T, Esterman A, May F, Phillips PA. A pragmatic $2 \times 2 \times 2$ factorial cluster randomized controlled trial of educational outreach visiting and case conferencing in palliative care-methodology of the Palliative Care Trial [ISRCTN 81117481]. Contemp Intern Med. 2006;27(1):83-100.

52. Whelan PJ, Walwyn R, Gaughran F, Macdonald A. Impact of the demand for 'proxy assent' on recruitment to a randomised controlled trial of vaccination testing in care homes. J Med Ethics. 2013;39(1):36-40.
53. Davies AN, Waghorn M, Webber K, Johnsen S, Mendis J, Boyle J. A cluster randomised feasibility trial of clinically assisted hydration in cancer patients in the last days of life. Palliat Med. 2018;32(4):733-43.

54. Rees $\mathrm{E}$, Hardy J. Novel consent process for research in dying patients unable to give consent. BMJ. 2003;327(7408):198.

55. Irwin S, Zurhellen C, Diamond L, Dunn L, Palmer B, Jeste D, Twamley E. Unrecognised cognitive impairment in hospice patients: a pilot study. Palliat Med. 2008;22(7):842-7.

56. Henwood TR, Keogh JW, Reid N, Jordan W, Senior HE. Assessing sarcopenic prevalence and risk factors in residential aged care: methodology and feasibility. J Cachexia Sarcopenia Muscle. 2014;5(3):229-36.

57. Wood F, Prout H, Bayer A, Duncan D, Nuttall J, Hood K, Butler CC. Consent, including advanced consent, of older adults to research in care homes: a qualitative study of stakeholders' views in South Wales. Trials. 2013;14(1):247.

58. Kleiderman E, Avard D, Black L, Diaz Z, Rousseau C, Knoppers BM. Recruiting terminally ill patients into non-therapeutic oncology studies: views of health professionals. BMC Med Ethics. 2012;13:33.

59. Buckles V, Powlishta K, Palmer J, Coats M, Hosto T, Buckley A, Morris J. Understanding of informed consent by demented individuals. Neurology. 2003;61(12):1662-6.

60. Black BS, Kass NE, Fogarty LA, Rabins PV. Informed consent for dementia research: the study enrollment encounter. IRB Ethics Hum Res. 2007;29(4):7-14.

61. Mittal D, Palmer BW, Dunn LB, Landes R, Ghormley C, Beck C, Golshan S, Blevins D, Jeste DV. Comparison of two enhanced consent procedures for patients with mild Alzheimer disease or mild cognitive impairment. Am J Geriatr Psychiatry. 2007;15(2):163-7.

62. Palmer BW, Harmell AL, Dunn LB, Kim SY, Pinto LL, Golshan S, Jeste DV. Multimedia aided consent for Alzheimer's disease research. Clin Gerontol. 2018:41(1):20-32.

63. Rubright J, Casarett DJ, Gur R, Xie SX, Karlawish J, Sankar P. A memory and organizational aid improves Alzheimer disease research consent capacity: results of a randomized, controlled trial. Am J Geriatr Psychiatry. 2010;18(12): 1124-32.

64. Olazarán J, Agüera-Ortiz L, Osorio RS, León-Salas B, Dobato JL, Cruz-Orduña I, González B, Valentí M, Gil-Ruiz N, Frades B. Promoting research in advanced dementia: early clinical results of the Alzheimer Center Reina Sofía Foundation. J Alzheimers Dis. 2012;28(1):211-22.

65. Hughes T, Castro RM. A processural consent methodology with people diagnosed with dementia. Qual Ageing Older Adults. 2015;16(4):222-34.

66. Kim SY, Karlawish JH, Kim HM, Wall IF, Bozoki AC, Appelbaum PS. Preservation of the capacity to appoint a proxy decision maker: implications for dementia research. Arch Gen Psychiatry. 2011;68(2):214-9.

67. Karlawish J, Kim SY, Knopman D, Van Dyck CH, James BD, Marson D. The views of Alzheimer disease patients and their study partners on proxy consent for clinical trial enrollment. Am J Geriatr Psychiatry. 2008;16(3):240-7.

68. Gainotti S, Imperatori SF, Spila-Alegiani S, Maggiore L, Galeotti F, Vanacore N, Petrini C, Raschetti R, Mariani C, Clerici F. How are the interests of incapacitated research participants protected through legislation? An Italian study on legal agency for dementia patients. PLoS One. 2010;5(6).

69. Aganwal MR, Ferran J, Ost K, WILSON KC. Ethics of informed consent'in dementia research — the debate continues. Int J Geriatric Psychiatry. 1996;11(9):801-6.

70. Cowdell F. Engaging older people with dementia in research: myth or possibility. Int J Older People Nursing. 2008;3(1):29-34.

71. Siminoff LA, Caputo M, Burant C. The promise of empirical research in the study of informed consent theory and practice. In: Hec Forum: 2004: Springer Science \& Business Media; 2004. p. 53.

72. Warner J, McCarney R, Griffin M, Hill K, Fisher P. Participation in dementia research: rates and correlates of capacity to give informed consent. J Med Ethics. 2007;34(3):167-70.

73. Black BS, Finucane T, Baker A, Loreck D, Blass D, Fogarty L, Phillips H, Hovanec L, Steele C, Rabins PV. Health problems and correlates of pain in nursing home residents with advanced dementia. Alzheimer Dis Assoc Disord. 2006;20(4):283-90.

74. Goodman C, Baron N, Machen I, Stevenson E, Evans C, Davies S, Illiffe S. Culture, consent, sosts and care homes: enabling older people with dementai to participate in research. Aging Ment Health. 2011;15(4):6.

75. Hanson LC, Gilliam R, Tae JL. Successful clinical trial research in nursing homes: the improving decision-making study. Clin Trials. 2010;7(6):735-43.

76. Baskin SA, Morris J, Ahronheim JC, Meier DE, Morrison RS. Barriers to obtaining consent in dementia research: implications for surrogate decisionmaking. J Am Geriatr Soc. 1998;46(3):287-90. 
77. Mitchell SL, Kiely DK, Jones RN, Prigerson H, Volicer L, Teno JM. Advanced dementia research in the nursing home: the CASCADE study. Alzheimer Dis Assoc Disord. 2006;20(3):166-75.

78. Ellis-Smith C, Higginson IJ, Daveson BA, Henson LA, Evans CJ. BuildCare: How can a measure improve assessment and management of symptoms and concerns for people with dementia in care homes? A mixed-methods feasibility and process evaluation of IPOS-Dem. PLoS One. 2018;13(7):e0200240.

79. Galeotti F, Vanacore N, Gainotti S, Izzicupo F, Menniti-Ippolito F, Petrini C, Chiarotti F, Chattat R, Raschetti R. How legislation on decisional capacity can negatively affect the feasibility of clinical trials in patients with dementia. Drugs Aging. 2012;29(8):607-14.

80. Kim SY, Kim HM, McCallum C, Tariot PN. What do people at risk for Alzheimer disease think about surrogate consent for research? Neurology. 2005;65(9):1395-401

81. Rikkert M, Van Den Bercken J, Ten Have H, Hoefnagels W. Experienced consent in geriatrics research: a new method to optimize the capacity to consent in frail elderly subjects. J Med Ethics. 1997;23(5):271-6.

82. Sudore RL, Landefeld CS, Williams BA, Barnes DE, Lindquist K, Schillinger D. Use of a modified informed consent process among vulnerable patients: a descriptive study. J Gen Intern Med. 2006;21(8):867-73.

83. Mason S, Barrow H, Phillips A, Eddison G, Nelson A, Cullum N, Nixon J. Brief report on the experience of using proxy consent for incapacitated adults. J Med Ethics. 2006;32(1):61-2.

84. Davies K, Collerton JC, Jagger C, Bond J, Barker SA, Edwards J, Hughes J, Hunt JM, Robinson L. Engaging the oldest old in research: lessons from the Newcastle 85+ study. BMC Geriatr. 2010;10:64.

85. Botker MT, Jorgensen MT, Stengaard C, Seidenfaden SC, Tarpgaard M, Granfeldt A, Mortensen TO, Grofte T, Friesgaard KD, Maerkedahl R, et al. Prehospital triage of patients suffering severe dyspnoea using N-terminal pro-brain natriuretic peptide, the PreBNP trial: a randomised controlled clinical trial. Eur Heart J Acute Cardiovasc Care. 2018;7(4):302-10.

86. Ford M, Kallen M, Richardson P, Matthiesen E, Cox V, Teng E, Cook K, Petersen N. Effect of social support on informed consent in older adults with Parkinson disease and their caregivers. J Med Ethics. 2008;34(1):41-7.

87. Thomalla G, Boutitie F, Fiebach JB, Simonsen CZ, Nighoghossian N, Pedraza S, Lemmens R, Roy P, Muir KW, Heesen C, et al. Effect of informed consent on patient characteristics in a stroke thrombolysis trial. Neurology. 2017; 89(13):1400-7.

88. Boxall L, Hemsley A, White N. Exploring recruitment issues in stroke research: a qualitative study of nurse researchers' experiences. Nurs Res. 2016;23(5):8-14.

89. Ali K, Roffe C, Crome P. What patients want: consumer involvement in the design of a randomized controlled trial of routine oxygen supplementation after acute stroke. Stroke. 2006;37(3):865-71.

90. Moran-Sanchez I, Luna A, Perez-Carceles MD. Assessment of capacity to consent to research among psychiatric outpatients: prevalence and associated factors. Psychiatry Q. 2016;87(1):89-105.

91. Harmell AL, Palmer BW, Jeste DV. Preliminary study of a web-based tool for enhancing the informed consent process in schizophrenia research. Schizophr Res. 2012;141(2-3):247-50.

92. Jeste DV, Palmer BW, Golshan S, Eyler LT, Dunn LB, Meeks T, Glorioso D, Fellows I, Kraemer H, Appelbaum PS. Multimedia consent for research in people with schizophrenia and normal subjects: a randomized controlled trial. Schizophr Bull. 2009;35(4):719-29.

93. Moser DJ, Reese RL, Hey CT, Schultz SK, Arndt S, Beglinger LJ, Duff KM, Andreasen NC. Using a brief intervention to improve decisional capacity in schizophrenia research; 2006.

94. Campbell MM, Susser E, Mall S, Mqulwana SG, Mndini MM, Ntola OA, Nagdee M, Zingela Z, Van Wyk S, Stein DJ. Using iterative learning to improve understanding during the informed consent process in a South African psychiatric genomics study. PLoS ONE. 2017;12(11):e0188466.

95. Carey E, Griffiths C. Recruitment and consent of adults with intellectual disabilities in a classic grounded theory research study: ethical and methodological considerations. Disabil Soc. 2017;32(2):193-212.

96. Ramerman L, Hoekstra PJ, de Kuijper G. Health-related quality of life in people with intellectual disability who use long-term antipsychotic drugs for challenging behaviour. Res Dev Disabil. 2018;75:49-58.

97. Ho P, Downs J, Bulsara C, Patman S, Hill AM. Addressing challenges in gaining informed consent for a research study investigating falls in people with intellectual disability. Br J Learn Disabil. 2018;46(2):92-100.

98. Hamilton J, Ingham B, McKinnon I, Parr JR, Tam LYC, Le Couteur A. Mental capacity to consent to research? Experiences of consenting adults with intellectual disabilities and/or autism to research. Br J Learn Disabil. 2017; 45(4):230-7.

99. Adamis D, Martin F, Treloar A, Macdonald A. Capacity, consent, and selection bias in a study of delirium. J Med Ethics. 2005;31(3):137-43.

100. Adamis D, Treloar A, Martin FC, Macdonald AJ. Ethical research in delirium: arguments for including decisionally incapacitated subjects. Sci Eng Ethics. 2010;16(1):169-74

101. Marcantonio ER, Bergmann MA, Kiely DK, Orav EJ, Jones RN. Randomized trial of a delirium abatement program for postacute skilled nursing facilities. J Am Geriatr Soc. 2010;58(6):1019-26.

102. Cole MG, McCusker J, Bellavance F, Primeau FJ, Bailey RF, Bonnycastle MJ, Laplante J. Systematic detection and multidisciplinary care of delirium in older medical inpatients: a randomized trial. Can Med Assoc J. 2002;167(7):753-9.

103. Mangset M, Forde R, Nessa J, Berge E, Wyller TB. I don't like that, it's tricking people too much...: acute informed consent to participation in a trial of thrombolysis for stroke. J Med Ethics. 2008;34(10):751-6.

104. Honarmand K, Belley-Cote EP, Ulic D, Khalifa A, Gibson A, McClure G, Savija N, Alshamsi F, D'Aragon F, Rochwerg B, et al. The deferred consent model in a prospective observational study evaluating myocardial injury in the intensive care unit. J Intensive Care Med. 2018;33(8):475-80.

105. Bolcic-Jankovic D, Clarridge BR, LeBlanc JL, Mahmood RS, Roman AM, Freeman BD. Exploring determinants of surrogate decision-maker confidence: an example from the ICU. J Empir Res Hum Res Ethics. 2014;9(4):76-85.

106. Levine AC, Barry MA, Agrawal P, Duber HC, Chang MP, Mackey JM, Hansoti B. Global Emergency Medicine Think Tank Clinical Research Working G: Global health and emergency care: overcoming clinical research barriers. Acad Emerg Med. 2017;24(4):484-93.

107. Smith OM, McDonald E, Zytaruk N, Foster D, Matte A, Clarke F, Fleury S, Krause K, McArdle T, Skrobik Y, et al. Enhancing the informed consent process for critical care research: strategies from a thromboprophylaxis trial. Intensive Crit Care Nurs. 2013;29(6):300-9.

108. Offerman SR, Nishijima DK, Ballard DW, Chetipally UK, Vinson DR, Holmes JF. The use of delayed telephone informed consent for observational emergency medicine research is ethical and effective. Acad Emerg Med. 2013;20(4):403-7.

109. Day TL, Bench SD, Griffiths PD. The role of pilot testing for a randomised control trial of a complex intervention in critical care. J Res Nurs. 2015;20(3): 167-78.

110. Bench S, Day T, Heelas K, Hopkins P, White C, Griffiths P. Evaluating the feasibility and effectiveness of a critical care discharge information pack for patients and their families: a pilot cluster randomised controlled trial. BMJ Open. 2015:5(11):e006852.

111. Higginson IJ, Rumble C, Shipman C, Koffman J, Sleeman KE, Morgan M, Hopkins P, Noble J, Bernal W, Leonard S, et al. The value of uncertainty in critical illness? An ethnographic study of patterns and conflicts in care and decision-making trajectories. BMC Anesthesiol. 2016;16:11.

112. Rouze A, Loridant S, Poissy J, Cornu M, Nseir S, Sendid B. Impact of the use of biomarkers on early discontinuation of empirical antifungal therapy in critically ill patients: a randomised controlled study. Med Mycol. 2018;56:S49.

113. Buckley JM, Irving AD, Goodacre S. How do patients feel about taking part in clinical trials in emergency care? Emerg Med J. 2016;33(6):376-80.

114. van Beinum A, Hornby L, Dhanani S, Ward R, Chambers-Evans J, Menon K. Feasibility of conducting prospective observational research on critically ill, dying patients in the intensive care unit. J Med Ethics. 2017;43(1):47-51.

115. Kamarainen A, Silfvast T, Saarinen S, Virta J, Virkkunen I. Conduct of emergency research in patients unable to give consent-experiences and perceptions of patients, their consent providing next of kin, and treating physicians following a prehospital resuscitation trial. Resuscitation. 2012;83(1):81-5.

116. Barrett KA, Ferguson ND, Athaide V, Cook DJ, Friedrich JO, McDonald E, Pinto R, Smith OM, Stevenson J, Scales DC. Surrogate decision makers' attitudes towards research decision making for critically ill patients. Intensive Care Med. 2012;38(10):1616-23.

117. Gong MN, Winkel G, Rhodes R, Richardson LD, Silverstein JH. Surrogate consent for research involving adults with impaired decision making: survey of Institutional Review Board practices. Crit Care Med. 2010;38(11):2146-54.

118. Burns KE, Magyarody NM, Duffett M, Nisenbaum R, Cook DJ. Attitudes of the general public toward alternative consent models. Am J Crit Care. 2011; 20(1):75-83.

119. Kim SY, Kim HM, Knopman DS, De Vries R, Damschroder L, Appelbaum PS Effect of public deliberation on attitudes toward surrogate consent for dementia research. Neurology. 2011;77(24):2097-104. 
120. Karlawish J, Rubright J, Casarett D, Cary M, Ten Have T, Sankar P. Older adults' attitudes toward enrollment of non-competent subjects participating in Alzheimer's research. Am J Psychiatry. 2009;166(2):182-8.

121. Bravo G, SY HK, Dubois M-F, Cohen CA, Wildeman SM, Graham JE. Surrogate consent for dementia research: factors influencing five stakeholder groups from the SCORES study. IRB Ethics Hum Res. 2013;35(4):1-11.

122. Bravo G, Duguet AM, Dubois MF, Delpierre C, Vellas B. Substitute consent for research involving the elderly: a comparison between Quebec and France. J Cross Cult Gerontol. 2008;23(3):239-53.

123. Farber NJ, Aboff BM, DeJoseph MR, Castellano J, Weiner J, Boyer EG. Physicians' understanding of consent requirements for phase I clinical trials in cognitively impaired or highly vulnerable populations. Account Res. 2004;11(1):63-78.

124. Kim SY, Kim HM, Langa KM, Karlawish JH, Knopman DS, Appelbaum PS. Surrogate consent for dementia research: a national survey of older Americans. Neurology. 2009;72(2):149-55.

125. De Vries R, Ryan KA, Stanczyk A, Appelbaum PS, Damschroder L, Knopman DS, Kim SY. Public's approach to surrogate consent for dementia research: cautious pragmatism. Am J Geriatr Psychiatry. 2013;21(4):364-72.

126. Karlawish JH, Knopman D, Clark CM, Morris JC, Marson D, Whitehouse PJ, Kawas CH. Informed consent for Alzheimer's disease clinical trials: a survey of clinical investigators. IRB. 2002;24(5):1-5.

127. Cahill M, Wichman A. Research involving persons with cognitive impairments: results of a survey of Alzheimer disease research centers in the United States. Alzheimer Dis Assoc Disord. 2000;14(1):20-7.

128. van der Vorm A, Vernooij-Dassen MJ, Kehoe PG, Olde Rikkert MG, van Leeuwen E, Dekkers WJ. Ethical aspects of research into Alzheimer disease A European Delphi Study focused on genetic and non-genetic research. J Med Ethics. 2009:35(2):140-4.

129. Peterson G, Wallin A. Alzheimer disease ethics-informed consent and related issues in clinical trials: results of a survey among the members of the Research Ethics Committees in Sweden. Int Psychogeriatr. 2003;15(2):157-70.

130. Garcia J, Evans J, Reshaw M. "Is there anything else you would like to tell us"- methodological issues int he use of free-text comments form postal surveys. Qual Quant. 2004;38:113-25.

131. Dickert NW, Hendershot KA, Speight CD, Fehr AE. Patients' views of consent in clinical trials for acute myocardial infarction: impact of trial design. J Med Ethics. 2017:43(8):524-9.

132. Olazaran J, Aguera-Ortiz L, Osorio RS, Leon-Salas B, Dobato JL, Cruz-Orduna I, Gonzalez B, Valenti M, Gil-Ruiz N, Frades B, et al. Promoting research in advanced dementia: early clinical results of the Alzheimer Center Reina Sofia Foundation. J Alzheimers Dis. 2012;28(1):211-22.

133. Gibbins J, Reid CM, Bloor S, Burcombe M, McCoubrie R, Forbes K. Overcoming barriers to recruitment in care of the dying research in hospitals. J Pain Symptom Manag. 2013;45(5):859-67.

134. European Parliament and of the Council of the European Union. Regulation (EU) No 536/2014 on clinical trials on medicinal products for human use, and repealing Directive 2001/20/EC. Off J Eur Union. 2014;57:L58-51-5820176.

135. Gysels MH, Evans C, Higginson IJ. Patient, caregiver, health professional and researcher views and experiences of participating in research at the end of life: a critical interpretive synthesis of the literature. BMC Med Res Methodol. 2012;12(1):123

136. Foster $C$. The ethics of medical research on humans. Cambridge: Cambridge University Press; 2001.

137. Koffman J, Stone K, Murtagh F. Ethics in palliative care research. In: Bruera EHI, von Gunten C, Morita T, editors. Textbook of Palliative Medicine. 2nd ed. London: CRC Press; 2015. p. 211-20

138. University of Leicester UoB. Adults lacking capacity - on-line toolkit: NRES; 2010

139. Koffman J, Yorganci E, Murtagh F, Yi D, Gao W, Barclay S, Pickles A, Higginson I, Johnson H, Wilson R. The AMBER care bundle for hospital inpatients with uncertain recovery nearing the end of life: the ImproveCare feasibility cluster RCT. Health Technol Assess. 2019;23(55):1.

140. Von Elm E, Altman DG, Egger M, Pocock SJ, Gøtzsche PC, Vandenbroucke JP. The Strengthening the Reporting of Observational Studies in Epidemiology (STROBE) statement: guidelines for reporting observational studies. Ann Intern Med. 2007;147(8):573-7.

141. Kaasa S, Dale O. Building up research in palliative care: an historical perspective and a case for the future. Clin Geriatr Med. 2005;21(1):81-92.

\section{Publisher's Note}

Springer Nature remains neutral with regard to jurisdictional claims in published maps and institutional affiliations.

Ready to submit your research? Choose BMC and benefit from:

- fast, convenient online submission

- thorough peer review by experienced researchers in your field

- rapid publication on acceptance

- support for research data, including large and complex data types

- gold Open Access which fosters wider collaboration and increased citations

- maximum visibility for your research: over $100 \mathrm{M}$ website views per year

At BMC, research is always in progress.

Learn more biomedcentral.com/submissions 